TECHNISCHE UNIVERSITÄT MÜNCHEN

DEPARTMENT CHEMIE

LEHRSTUHL FÜR BIOCHEMIE

\title{
Structural and functional characterization of the immunoproteasome
}

\author{
Eva Maria Huber
}

Vollständiger Abdruck der von der Fakultät für Chemie der Technischen Universität München zur Erlangung des akademischen Grades eines Doktors der Naturwissenschaften (Dr. rer. nat.) genehmigten Dissertation.

Vorsitzender:

Prüfer der Dissertation:
Univ.-Prof. Dr. L. Hintermann

1. Univ.-Prof. Dr. M. Groll

2. apl. Prof. Dr. Dr. h.c. R. Huber (i. R.)

3. Univ.-Prof. Dr. Dr. h.c. B. Rieger

Die Dissertation wurde am 06.02.2013 bei der Technischen Universität München eingereicht und durch die Fakultät für Chemie am 27.03.2013 angenommen. 

Meiner Familie 



\section{Table of Contents}

Summary 8

3.1 Intracellular protein degradation and antigen presentation ................................. 12

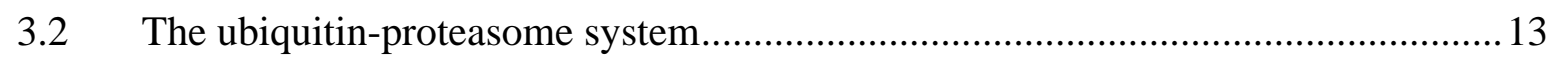

3.2.1 Polyubiquitylation - a signal for degradation.................................................. 13

3.2.2 Subunit composition and architecture of proteasomes ..................................... 14

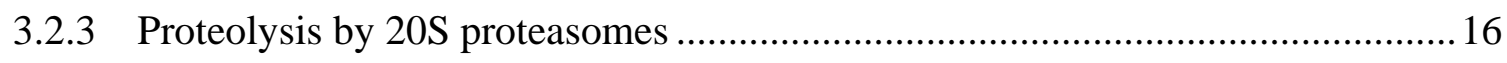

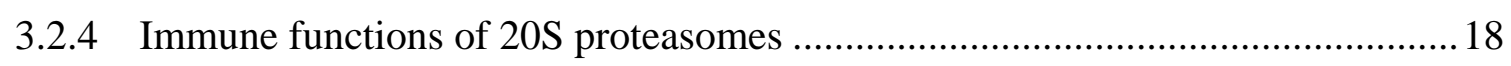

3.3 20S proteasomes: validated and emerging drug targets ..................................... 19

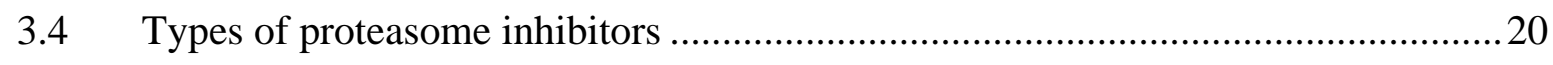

3.5 Clinically relevant proteasome inhibitors...................................................... 21

3.6 Subunit-specific proteasome inhibitors and their therapeutic potential ..................23

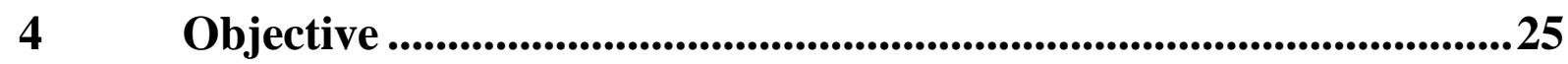

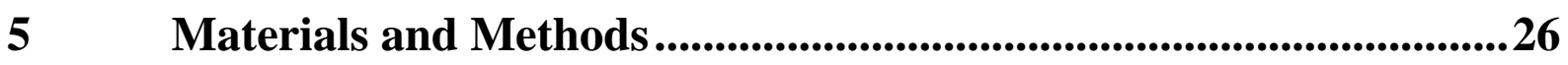

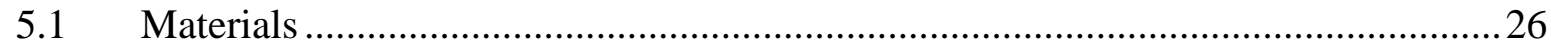

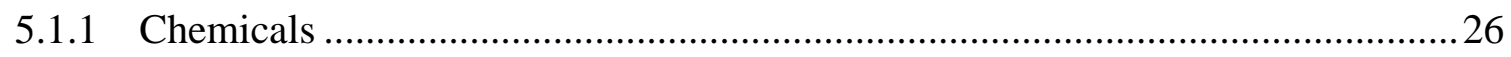

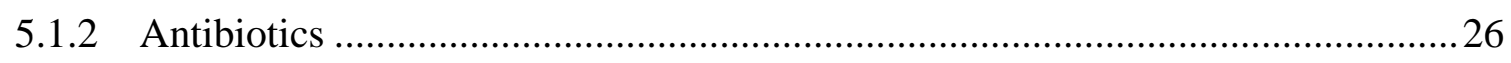

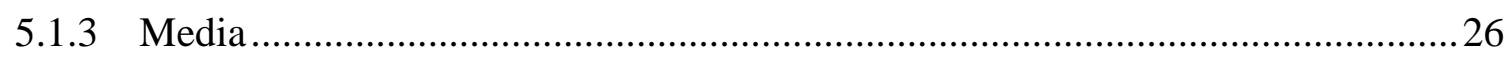

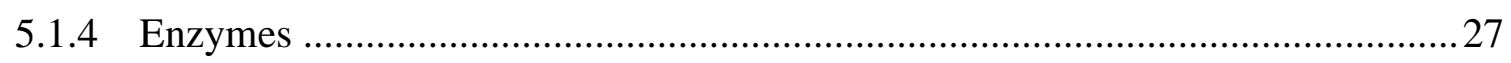

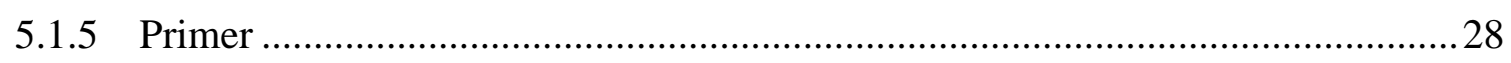

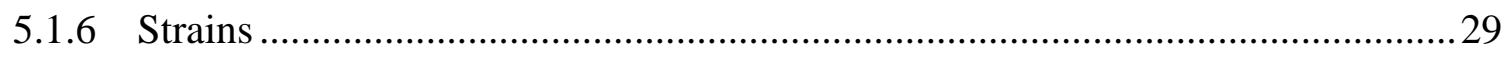

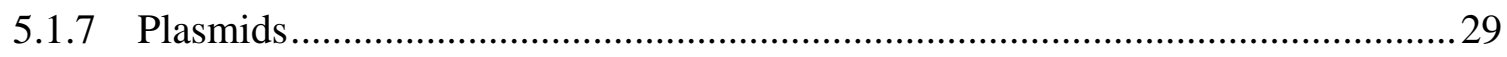

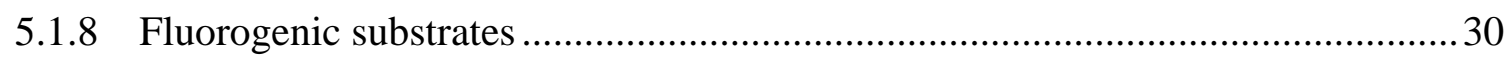

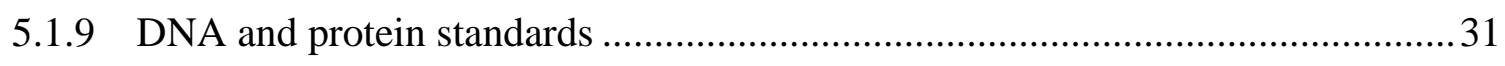

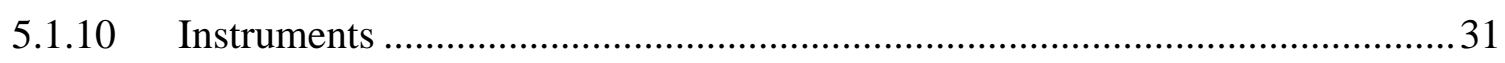

5.1.11 Computer software and bioinformatics tools .............................................. 33

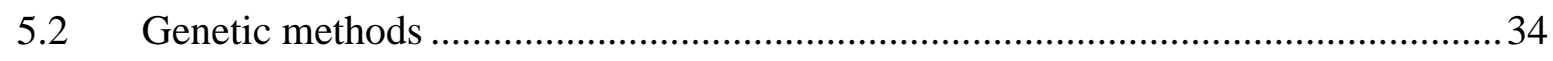


5.2.1 Cultivation and long-term storage of Escherichia coli ..................................... 34

5.2.2 Cultivation and long-term storage of Saccharomyces cerevisiae ........................ 34

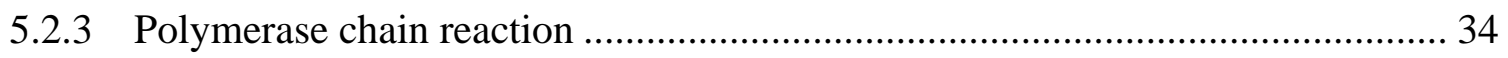

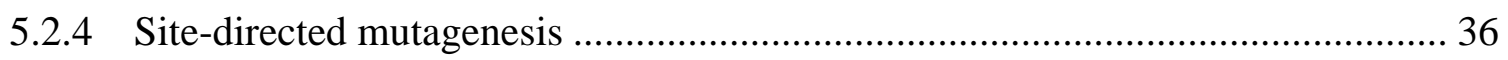

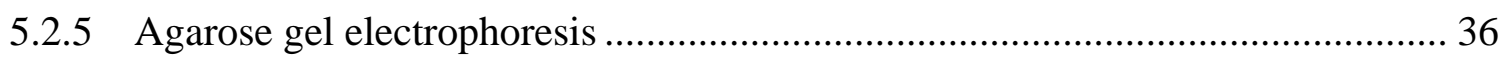

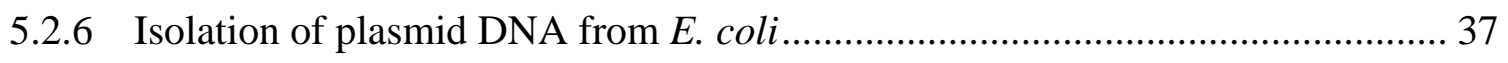

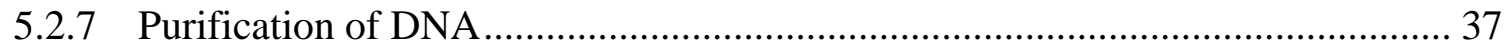

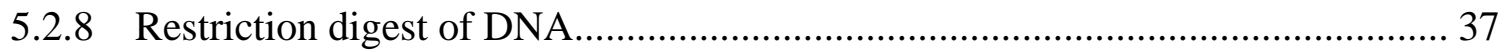

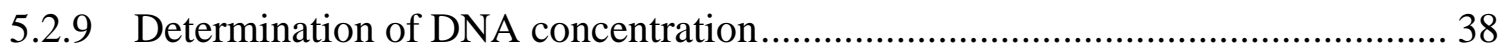

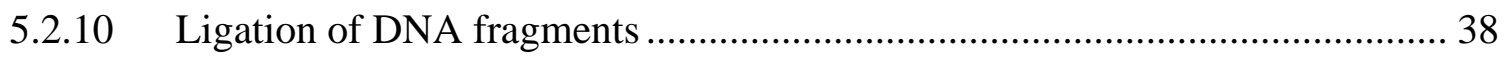

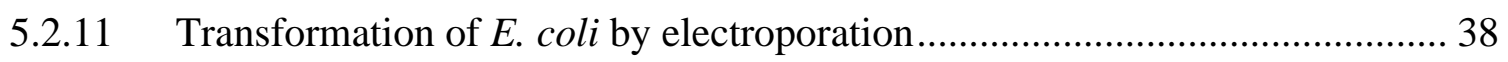

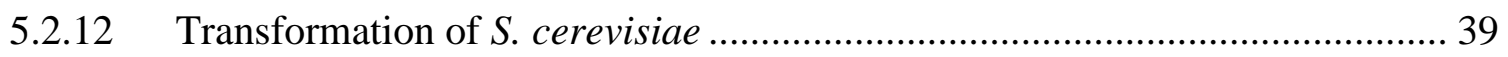

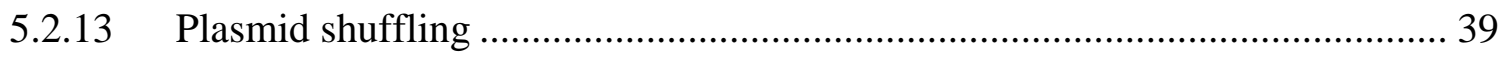

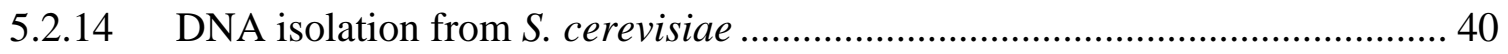

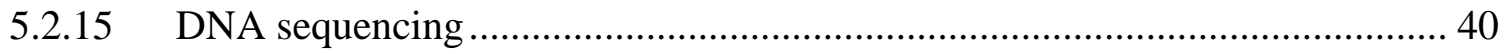

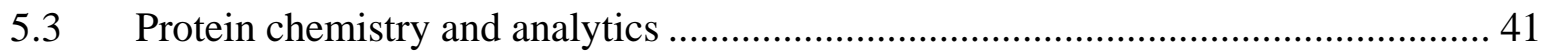

5.3.1 Purification of the yeast $20 \mathrm{~S}$ proteasome ..................................................... 41

5.3.2 Purification of the murine immuno- and constitutive 20S proteasome ............... 41

5.3.3 SDS polyacrylamide gel electrophoresis ...................................................... 42

5.3.4 Determination of protein concentration ....................................................... 43

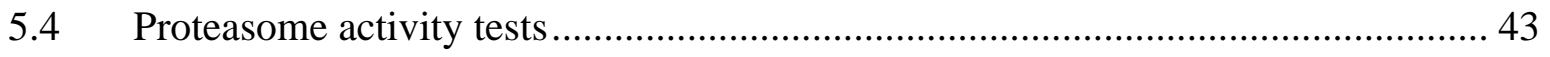

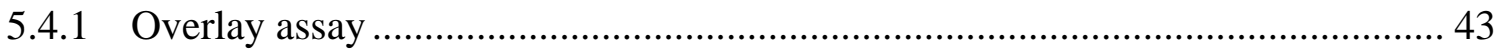

5.4.2 Fluorescence activity test and determination of $\mathrm{IC}_{50}$ values .............................. 44

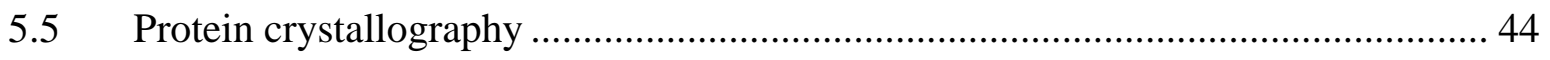

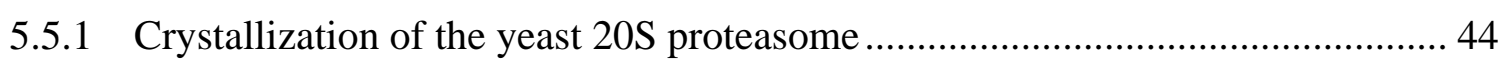

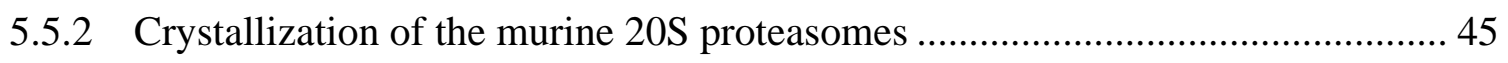

5.5.3 Data collection, processing and structure determination .................................. 45

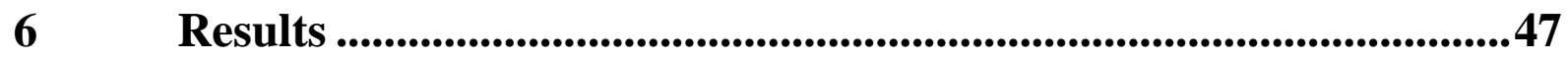

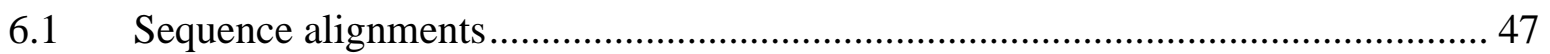

6.2 X-ray structures of the mouse 20S immuno- and constitutive proteasome ............ 49

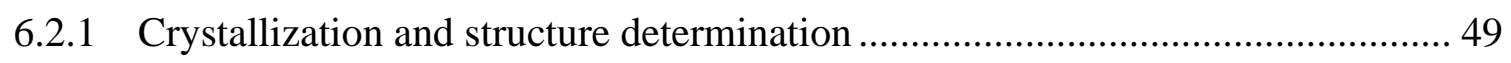

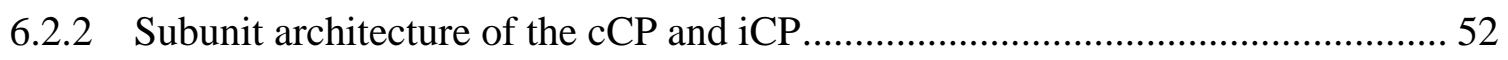

6.2.3 Structural analysis of the substrate binding channels ..................................... 53 


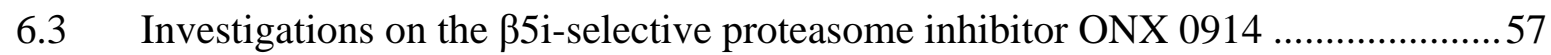

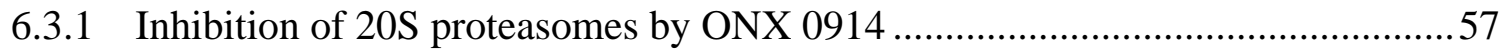

6.3.2 Proteasome core particles in complex with ONX 0914 .....................................57

6.3.3 Molecular basis for the subunit selectivity of ONX 0914 _...............................59

6.3.4 Structural analysis of the epoxyketone reaction mechanism..............................62

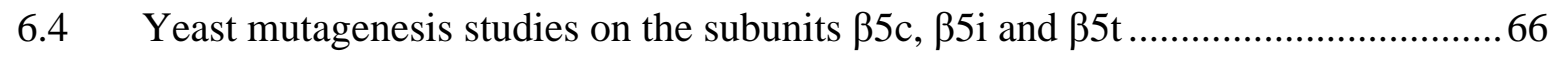

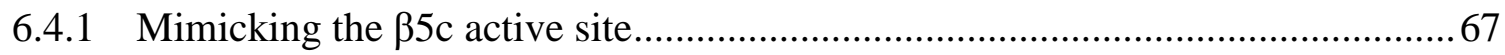

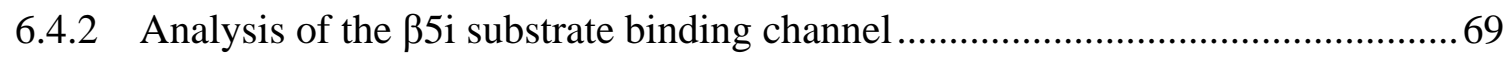

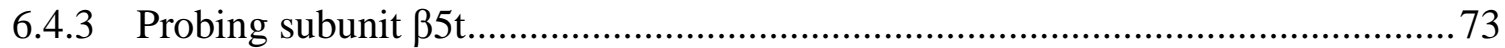

$7 \quad$ Discussion.......................................................................................76

7.1 Structural and functional differences between the three types of CPs ...................76

7.2 Guidelines for the rational design of CP-selective inhibitors ..................................78

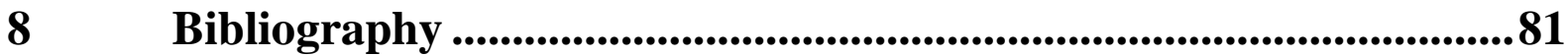

$9 \quad$ Appendix $. . . \ldots \ldots \ldots \ldots \ldots \ldots \ldots \ldots \ldots \ldots \ldots \ldots \ldots \ldots \ldots \ldots \ldots \ldots \ldots \ldots \ldots \ldots \ldots \ldots \ldots \ldots \ldots \ldots \ldots \ldots \ldots \ldots \ldots \ldots \ldots \ldots . . . \ldots 90$

10 Abbreviations ....................................................................96

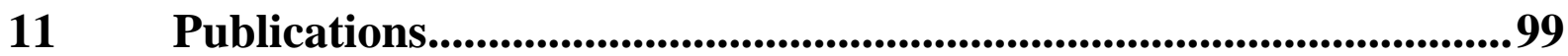

12 Acknowledgement ...........................................................................100 


\section{Summary}

$20 \mathrm{~S}$ proteasomes are destructive molecular machines that break down proteins into small peptides. Based on their task to regulate intracellular protein homoeostasis and to eliminate aberrant polypeptides, proteasomes are essential for cell division and differentiation. Hence, inhibition of the main proteasome types, the constitutive proteasome and the immunoproteasome, represents a successful therapeutic strategy against cancers. Moreover, selective inhibition of the immunoproteasome, playing also a crucial role in antigen processing, is currently emerging as a medicinal rationale for autoimmune disorders and chronic inflammations. However, so far the development of selective compounds was hampered by the lack of structural data on the immunoproteasome.

The present thesis describes the structural and functional characterization of the constitutive proteasome and the immunoproteasome from mouse, which will facilitate structure-based development of novel drugs. Using X-ray crystallography, the 3D-structures of both murine proteasome types have been determined at atomic resolution and the differences between the proteolytically active subunits of the immunoproteasome ( $\beta 1 \mathrm{i}, \beta 2 \mathrm{i}$ and $\beta 5 \mathrm{i})$ and its constitutive counterpart $(\beta 1 \mathrm{c}, \beta 2 \mathrm{c}$ and $\beta 5 \mathrm{c}$ ) could be revealed. As the substrate specificity pockets of the active sites differ in their size and polarity, they give rise to distinct cleavage and inhibitor preferences. In this regard the $\beta 1 \mathrm{i}$ and $\beta 5 \mathrm{i}$ subunits of the immunoproteasome evolved to generate antigens with hydrophobic C-terminal anchor residues that bind with high affinity to major histocompatibility class I receptors to control immune responses.

In addition, ligand complex structures of both proteasome types with the first $\beta 5 \mathrm{i}$-specific compound, the tripeptide $\alpha^{\prime}, \beta$ 'epoxyketone ONX 0914 (PR-957) provide explanation for its subunit selectivity. While the accessibility of ONX 0914 to the active sites of $\beta 1 \mathrm{c}$ and $\beta 1 \mathrm{i}$ is either electrostatically or sterically hampered, the $\beta 2 \mathrm{c}$ und $\beta 2 \mathrm{i}$ substrate binding channels cannot provide enough stabilization for the ligand. Furthermore, binding of ONX 0914 significantly enlarges the $\mathrm{S} 1$ pocket of the $\beta 5 \mathrm{c}$ active site and shifts the adjacent peptide backbone by up to $1.7 \AA$. In contrast, these structural changes do not occur in subunit $\beta 5 \mathrm{i}$ of the immunoproteasome. Different side chain conformations of Met45 create a spacious S1 site in subunit $\beta 5 \mathrm{i}$ and a significantly smaller one in $\beta 5 \mathrm{c}$, thereby facilitating binding of ONX 0914 to the $\beta 5 \mathrm{i}$ active site compared to $\beta 5 \mathrm{c}$. In addition, the ligand complex structures disclose that the binding mechanism of peptidic compounds is identical in all proteasome subunits, including the formation of an antiparallel $\beta$ sheet. The selectivity of peptidomimetics therefore solely depends on the interactions of the ligand's side chains with the substrate 
binding channel. Time-resolved examination of the mode of action of ONX 0914 using the yeast $20 \mathrm{~S}$ proteasome elucidated that apart from the electrophilic warhead particularly the P1 site determines the affinity of compounds for the active sites of the protease.

Sequence differences between the $\beta 5$ subunits of the constitutive proteasome and the immunoproteasome were investigated by mutagenesis of the yeast $20 \mathrm{~S}$ proteasome. Furthermore, the structurally uncharacterized substrate binding channel of subunit $\beta 5 t$ that assembles into the vertebrate-specific thymoproteasome was mimicked in yeast. These mutant proteasomes elucidate the impact of single amino acid exchanges on the S1 pocket architecture and the affinity of proteasome inhibitors.

The structural and functional characterization of the murine 20S proteasomes described herein now supports the development of novel selective drugs for the distinct proteasome types and their active sites, which are of considerable therapeutic potential against cancers and autoimmune diseases. 


\section{Zusammenfassung}

Das 20S Proteasom ist eine molekulare Maschine, die Proteine in Peptide zerlegt. Aufgrund seiner Aufgabe das intrazelluläre Proteingleichgewicht $\mathrm{zu}$ kontrollieren und nicht mehr funktionsfähige Proteine zu recyceln, ist das Proteasom unabdingbar für die Zellteilung und Zelldifferenzierung. Inhibition der beiden Haupttypen des Proteasoms, des konstitutiven Proteasoms und des Immunoproteasoms, stellt deshalb eine wirksame Therapiemöglichkeit von Krebserkrankungen dar. Zudem deuten neuere Studien darauf hin, dass eine selektive Hemmung des Immunoproteasoms, das eine zentrale Rolle für die Prozessierung von Antigenen spielt, von therapeutischem Nutzen für die Behandlung von chronischen Entzündungen und Autoimmunerkrankungen sein könnte. Jedoch war bisher eine gezielte Entwicklung von selektiven Hemmstoffen aufgrund fehlender Strukturinformation über das Immunoproteasom erschwert.

Die vorliegende Arbeit beschreibt die strukturelle und funktionelle Charakterisierung des konstitutiven Proteasoms und des Immunoproteasoms der Maus und erleichtert nun die struktur-basierte Entwicklung von neuen Medikamenten. Mit Hilfe der Röntgenkristallographie konnten die atomaren Strukturen der beiden murinen Proteasomtypen bestimmt werden und die Unterschiede zwischen den proteolytisch aktiven Untereinheiten des Immunoproteasoms ( $\beta 1 \mathrm{i}, \beta 2 \mathrm{i}$ und $\beta 5 \mathrm{i}$ ) sowie des konstitutiven Proteasoms ( $\beta 1 \mathrm{c}, \beta 2 \mathrm{c}$ und $\beta 5 \mathrm{c}$ ) herausgearbeitet werden. Da sich die Substratbindetaschen der einzelnen aktiven Zentren sowohl in ihrer Größe als auch Polarität unterscheiden, ermöglichen sie verschiedene Substrat- und Inhibitorspezifitäten. Mit den $\beta 1 \mathrm{i}$ und $\beta 5 \mathrm{i}$ Untereinheiten ist das Immunoproteasom dafür optimiert Epitope mit apolaren C-terminalen Enden zu generieren. Diese binden mit hoher Affinität an die Haupthistokompatibilitäts-Rezeptoren der Klasse I und kontrollieren somit Immunantworten. Zudem erklären Ligandenkomplexstrukturen beider Proteasomtypen mit dem ersten $\beta 5$ i-spezifischen Inhibitor, dem tripeptidischen $\alpha^{‘}, \beta$ 'Epoxyketon ONX 0914 (PR-957), dessen Untereinheiten-Selektivität. Der Zugang von ONX $0914 \mathrm{zu}$ den aktiven Zentren der $\beta 1 \mathrm{c}$ und $\beta 1 \mathrm{i}$ Untereinheiten ist elektrostatisch beziehungsweise sterisch behindert und in den Untereinheiten $\beta 2 \mathrm{c}$ und $\beta 2 \mathrm{i}$ kann der Ligand nur unzureichend stabilisiert werden. Darüber hinaus verursacht die Bindung von ONX 0914 an das aktive Zentrum der $\beta 5$ c Untereinheit eine starke Vergrößerung der S1 Spezifitätstasche sowie eine Verschiebung des angrenzenden Peptidkettenverlaufs um bis zu $1.7 \AA$. Im Gegensatz dazu bewirkt ONX 0914 keinerlei Veränderungen im Substratbindekanal der $\beta 5$ i Untereinheit des Immunoproteasoms. Unterschiedliche Seitenkettenkonformationen von 
Met45 in der $\beta 5 \mathrm{i}$ und $\beta 5$ c Untereinheit machen die S1 Tasche der $\beta 5 \mathrm{i}$ Untereinheit im Vergleich zu $\beta 5 \mathrm{c}$ geräumiger, sodass die Bindung von ONX0914 in den $\beta 5 \mathrm{i}$ Substratbindekanal leichter als an das $\beta 5 \mathrm{c}$ aktive Zentrum erfolgen kann. Weiterhin zeigen die Ligandenkomplexstrukturen auf, dass der Bindemechanismus des peptidischen Inhibitors für alle Proteasomuntereinheiten des Immuno- und des konstitutiven Proteasoms identisch über die Formierung eines anti-parallelen $\beta$ Faltblattes verläuft. Somit wird die Selektivität von Peptidomimetika einzig von den Interaktionen der Liganden-Seitenketten mit dem Substratbindekanal bestimmt. Zeitaufgelöste Untersuchungen zum Reaktionsmechanismus von ONX 0914 am 20S Proteasom von Saccharomyces cerevisiae zeigen auf, dass abgesehen von der elektrophilen Kopfgruppe, insbesondere die P1 Seitenkette von Inhibitoren deren Affinität für die proteolytisch aktiven Zentren bestimmt.

Die Sequenzunterschiede in den Proteolysezentren der $\beta 5$ Untereinheiten des konstitutiven Proteasoms und des Immunoproteasoms wurden mittels Mutagenese des 20S Proteasoms aus $S$. cerevisiae untersucht. Darüber hinaus wurde der Substratbindekanal der $\beta 5 t$ Untereinheit, die in das sogenannte Thymusproteasom von Vertebraten eingebaut wird und strukturell nicht charakterisierte ist, in Hefe rekonstituiert. Diese Mutanten klären die Bedeutung einzelner Aminosäureaustausche für die Architektur der S1 Taschen sowie für die Affinität von Proteasominhibitoren.

Die hier erläuterte strukturelle und funktionelle Charakterisierung der 20S Proteasome aus der Maus unterstützt die Entwicklung neuer selektiver Hemmstoffe für die einzelnen Proteasomtypen und -untereinheiten, welche gegenwärtig von hohem therapeutischen Potential für die Behandlung von Krebs- und Autoimmunerkrankungen sind. 


\section{Introduction}

\subsection{Intracellular protein degradation and antigen presentation}

All proteins undergo a life cycle, starting from their synthesis at ribosomes and ending with their degradation to peptides and single amino acids. Thereby, the building blocks for the de novo synthesis of polypeptides are recycled and in addition important cellular functions are controlled. These include protein homoeostasis, cell proliferation, signal transduction and antigen production ${ }^{[1]}$. The lysosomal and the non-lysosomal protein degradation pathways process the majority of intracellular self and nonself proteins. In lysosomes proteases termed cathepsins unselectively degrade proteins. The resulting peptide fragments can be loaded on major histocompatibility complex class (MHC) II receptors and presented on the cell surface to immune cells ${ }^{[2]}$. In contrast, hydrolysis of more than $90 \%$ of all cytosolic proteins is carried out by the $26 \mathrm{~S}$ proteasome - the central player of the non-lysosomal protein degradation pathway. The 26S proteasome, a multicatalytic ATP-dependent protease located in the cytosol and the nucleus, selectively cuts polyubiquitylated proteins to peptides of diverse lengths ${ }^{[3]}$. While most of these peptides are further decomposed to single amino acids, a fraction escapes this fate and serves as antigens for the immune system in vertebrates. Prior or after N-terminal trimming to $8-11$ amino acids by aminopeptidases ${ }^{[4]}$ putative epitopes transit into the endoplasmic reticulum (ER) by the transporter associated with antigen processing (TAP), a member of the ATP-binding cassette transporter family ${ }^{[5]}$. In the ER peptides can associate with the binding cleft of nascent MHC I receptors. The peptide's affinity for the MHC I complex largely depends on its C-terminal anchor residue ${ }^{[6]}$, which is determined by the cleavage specificities of the proteasome. Only stable receptor:ligand complexes adopt a mature structure and are transported in vesicles to the cell membrane for their exposure to the extracellular environment. Surveying effector cells of the immune system scan the peptide cargos of both MHC I and II receptor proteins for their origin and cells that carry bacterial or viral peptides on their cell surface are identified and eliminated by cytotoxic $\mathrm{T}$ lymphocytes to prevent an infection ${ }^{[7]}$. Hence, by shaping the antigenic pool of peptides the proteasome constitutes a key component of the adaptive immune system of vertebrates. 


\subsection{The ubiquitin-proteasome system}

\subsubsection{Polyubiquitylation - a signal for degradation}

Posttranslational modifications such as ubiquitylation control the subcellular localization, signal transduction, enzymatic activity and stability of eukaryotic proteins. Ubiquitin itself is a highly conserved polypeptide of $8.5 \mathrm{kDa}$ and 76 amino acids and its covalent linkage to proteins is catalysed by the sequential action of three classes of enzymes, termed ubiquitinactivating enzyme (E1), ubiquitin-conjugating enzyme (E2) and ubiquitin ligase (E3) ${ }^{[3 \mathrm{~b}]}$. The E1 enzyme activates the C-terminal glycine residue of ubiquitin in an ATP-dependent manner under the release of pyrophosphate. Subsequent attachment of the adenylated ubiquitin to the active site Cys residue of the E1 enzyme yields a thioester and an AMP molecule. Next, ubiquitin is conjugated to the catalytic Cys of an ubiquitin-conjugating enzyme (E2) and finally, an E3 ligase creates an isopeptide bond between the ubiquitin's C-terminus and the $\varepsilon$ amino group of a lysine side chain in the substrate protein (Figure 1) ${ }^{[3 b]}$. Selectivity of the ubiquitylation reaction is ensured by a myriad of E3 ligases that binds to recognition sites in their target proteins either directly or via adaptor proteins. Commonly, proteins that carry a chain of at least four ubiquitin molecules connected via Lys48 are targeted for degradation by the $26 \mathrm{~S}$ proteasome ${ }^{[8]}$.

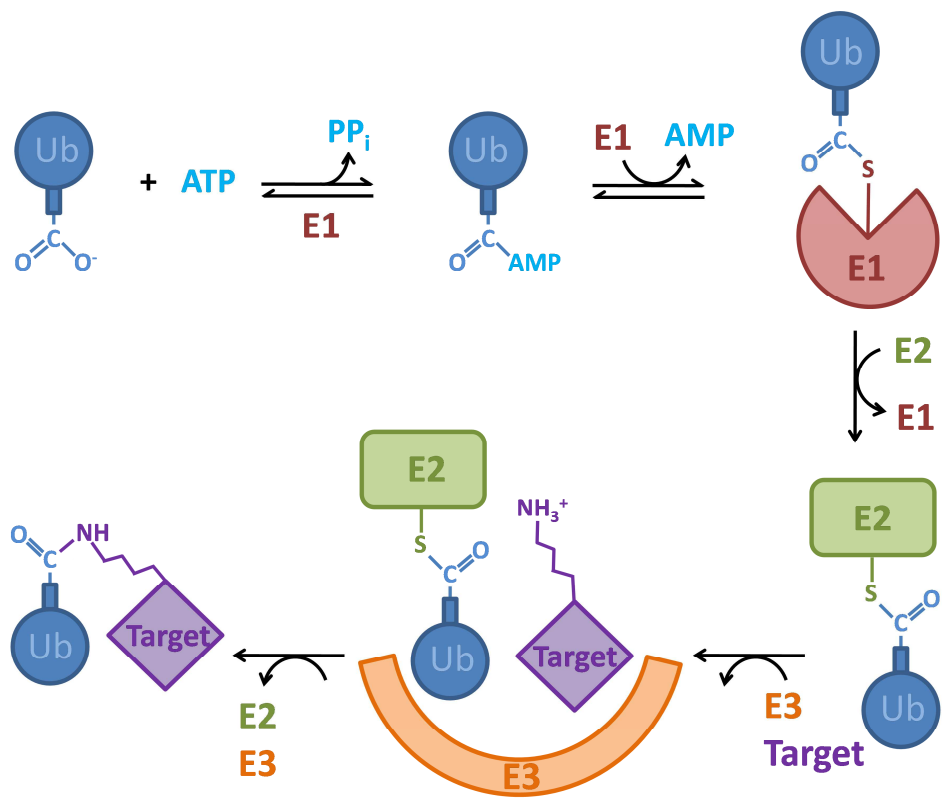

Figure 1 Ubiquitin activation and linkage to substrate proteins. Adenylation of ubiquitin by an E1 enzyme is followed by its transfer to an E2 ubiquitin-conjugating enzyme. An E3 ligase covalently attaches ubiquitin to a lysine residue in the target protein.

Adapted from Berg and Stryer, 2002 $2^{[9]}$. 


\subsubsection{Subunit composition and architecture of proteasomes}

The $26 \mathrm{~S}$ proteasome is a molecular machine of approximately $2.5 \mathrm{MDa}$, consisting of a cylindrical 20S core particle (CP) and two 19S regulatory complexes associated on both ends. The 19S cap binds polyubiquitylated substrates, cleaves off their ubiquitin molecules, unfolds and finally translocates client proteins into the proteolytic $20 \mathrm{~S}$ chamber ${ }^{[10]}$. In contrast to the 19S complex whose atomic structure has not yet been resolved, the core of the proteasome has been extensively characterized at the molecular level, leading to the following current understanding of its evolution and functioning.

The barrel-shaped CP is present in all three kingdoms of life, bacteria, archaea and eukaryotes. Bacteria except for actinomycetes harbour CP-like complexes termed heat-shock locus (Hsl) V that consist of two homohexameric rings of a proteolytically active subunit ${ }^{[11]}$. The CP of actinomycetes, archaea and eukaryotes adopts a more complex quarternary structure. The X-ray structures of the $\mathrm{CP}$ from the archaeon Thermoplasma acidophilum ${ }^{[12]}$ and the eukaryote $S$. cerevisiae ${ }^{[13]}$ proved that the protease is a cylinder of $148 \AA$ in length and $113 \AA$ in width with a molecular mass of approximately $720 \mathrm{kDa}$ and that it consists of two different types of subunits termed $\alpha$ and $\beta$. Both monomers are assembled in four homoheptameric rings that are stacked in an $\alpha \beta \beta \alpha$ stoichiometry around a central pore. Due to its two-fold rotational symmetry the $\mathrm{CP}$ comprises two identical halves (Figure 2) ${ }^{[12-13]}$. Archaeal 20S proteasomes incorporate only one type of $\alpha$ and one type of $\beta$ subunit $^{[12]}$. By contrast, eukaryotic proteasomes contain seven different $\alpha$ (1-7) and seven different $\beta$ (1-7) subunits (Figure 2) ${ }^{[13]}$, which occupy unique positions within the CP. All known $\alpha$ and $\beta$ subunits share the same tertiary structure of two antiparallel five-stranded $\beta$ sheets being flanked by $\alpha$ helices and probably evolved from a common ancestor (Figure $2 \mathrm{~A})^{[12,14]}$.

Several functions have been attributed to the $\alpha$ subunits. Their intrinsic ability to form a heptameric ring is pivotal for the assembly of the $\beta$ subunits and the formation of the $\mathrm{CP}$. Furthermore, so-called shuttling sequences in the $\alpha$ subunits enable the import of the $\mathrm{CP}$ into the nucleus and its re-export ${ }^{[15]}$. The $\alpha$ subunits also form the entry gate to the interior of the $\mathrm{CP}^{[16]}$. In particular, the $\mathrm{N}$-terminal extensions of the subunits $\alpha 2, \alpha 3$ and $\alpha 4$ close the barrelshaped CP on both ends and thereby abolish uncontrolled degradation of intracellular proteins ${ }^{[12-13]}$. Docking of regulatory and adapter complexes such as the 11S (PA28), 19S and PA200 (Blm10) particles onto the $\alpha$ ring rearranges the N-termini of the $\alpha$ subunits and enables access to the proteolytically active sites that are sequestered in the $\beta$ rings ${ }^{[17]}$. The $\beta$ subunits are synthesized as inactive precursor proteins with $\mathrm{N}$-terminal propeptides of up to 75 amino acids. These propeptides are involved in CP assembly assisted by the proteasome 
maturation factor Ump1 (ubiquitin-mediated proteolysis 1$)^{[18]}$ and protect the catalytic active sites from inactivating $\mathrm{N} \alpha$ acetylation prior to proteasome maturation ${ }^{[19]}$. In the final step of proteasome assembly the propeptides are removed by intramolecular autolysis, thereby exposing the proteolytically active Thr1 residues and yielding a proteasome particle that can cut polypeptides down to size ${ }^{[12,20]}$. Remarkably, in eukaryotes only three out of the seven different $\beta$ subunits, namely $\beta 1, \beta 2$ and $\beta 5$, are proteolytically active as $\mathrm{N}$-terminal nucleophile (Ntn) threonine hydrolases ${ }^{[13]}$.

A

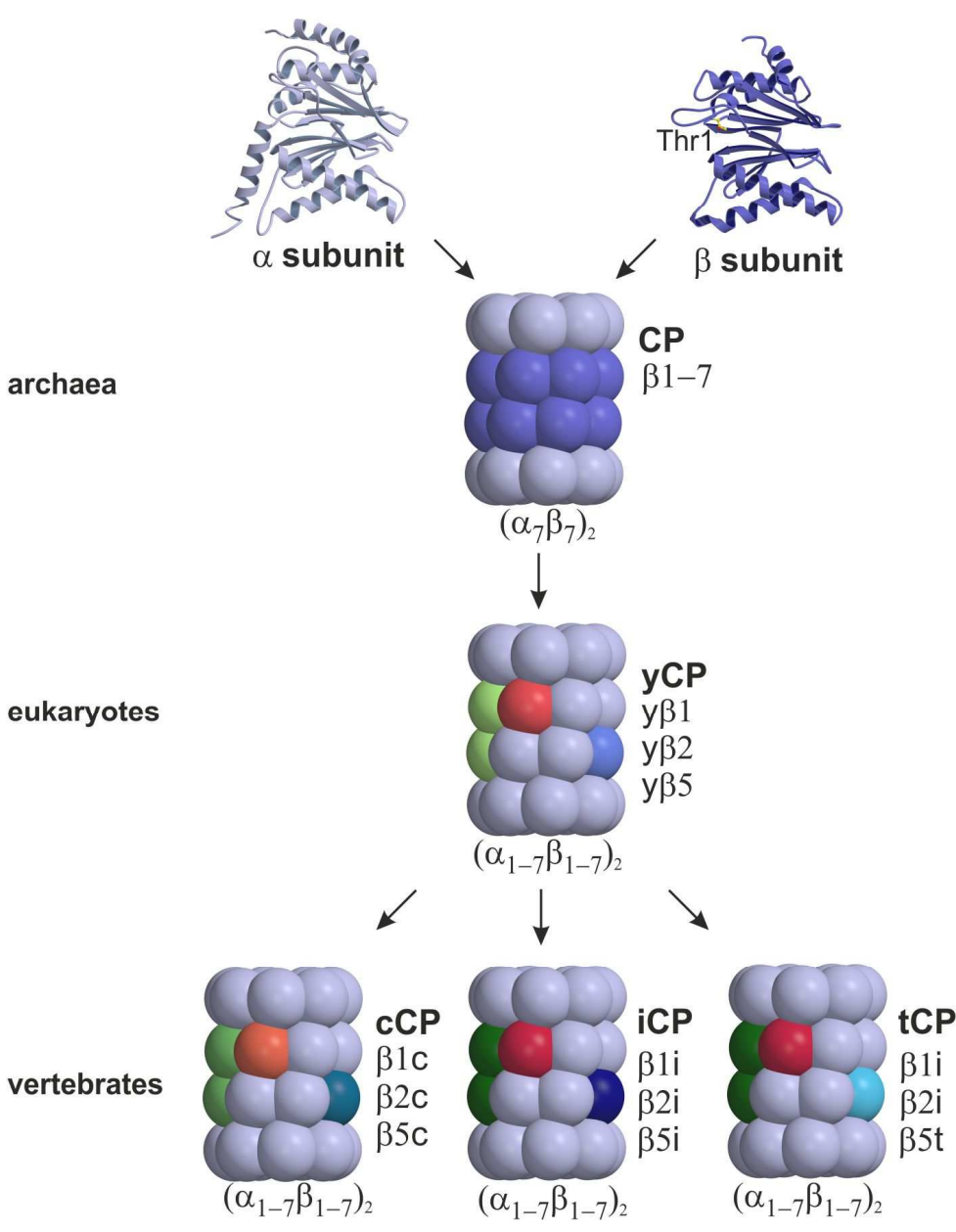

B
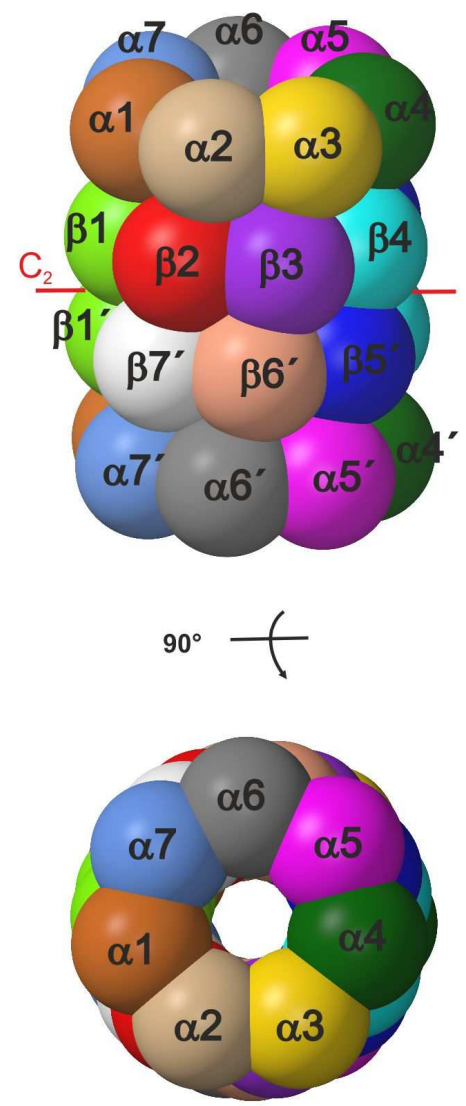

Figure 2 Evolution of $20 \mathrm{~S}$ proteasomes. (A) Archaeal CPs are assembled of two different types of monomers, namely inactive $\alpha$ (grey) and proteolytically active $\beta$ subunits (blue). Both presumably originated from a common but unknown precursor protein ${ }^{[14]}$. Eukaryotes such as S. cerevisiae encode seven different $\alpha$ and seven different $\beta$ subunits, but only the $\beta$ subunits $\beta 1$ (green), $\beta 2$ (red) and $\beta 5$ (blue) are enzymatically active ${ }^{[13]}$. Their inactive $\alpha$ and $\beta$ subunits are coloured in grey. In vertebrates three classes of $20 \mathrm{~S}$ proteasomes evolved. The constitutive proteasome $(\mathrm{cCP})$, the immunoproteasome $(\mathrm{iCP})$ and the thymoproteasome $(\mathrm{tCP})$ have distinct sets of catalytic $\beta$ subunits and thus, are implemented in different biological pathways ${ }^{[13,21]}$. (B) Side and top view of the eukaryotic CP. The $\mathrm{C}_{2}$ symmetry of the proteasome particle is indicated as a red rod.

Adapted from Huber et al., 2012 $2^{[22]}$. 
Whereas primitive eukaryotes like baker's yeast bear only one type of $\mathrm{CP}^{[13]}$, vertebrates express three classes of $\mathrm{CPs}^{[21 c, 23]}$ : the constitutive proteasome (cCP), the immunoproteasome (iCP) and the thymoproteasome (tCP). The $\mathrm{cCP}$ comprises the catalytic active constitutive (c) subunits $\beta 1 \mathrm{c}(\mathrm{Y}, P S M B 6), \beta 2 \mathrm{c}(\mathrm{Z}, P S M B 7)$ and $\beta 5 \mathrm{c}(\mathrm{X}, P S M B 5)$ and represents the prevailing proteasome species in cells of non-haematopoietic origin. In contrast, in immune cells such as lymphocytes and monocytes the $\mathrm{iCP}$ is predominant ${ }^{[23]}$ (Figure 2A). Nonetheless, expression of the iCP can also be induced in non-immune tissues by proinflammatory cytokines such as interferon (IFN)- $\gamma$ and tumour necrosis factor (TNF)- $\alpha^{[21 b, 24]}$. As the three proteolytically active immuno (i) subunits $\beta 1 \mathrm{i}$ (LMP2, low molecular weight protein 2, PSMB9), $\beta 2 \mathrm{i}$ (MECL1, multicatalytic endopeptidase complex-like-1, PSMB10) and $\beta 5$ i (LMP7, PSMB8) are preferentially assembled into CPs, mainly iCPs are formed de novo upon cytokine release $^{[21 b, 25]}$. Apart from the i subunits IFN- $\gamma$ also triggers the expression of the $11 \mathrm{~S}$ adaptor complex PA28 $\alpha \beta^{[26]}$. This particle stimulates proteasome activity and is essential for the generation of certain antigenic peptides ${ }^{[27]}$. Furthermore, PA28 $\alpha \beta$ has been suggested to target iCPs to the TAP in the ER membrane in order to directly translocate the generated peptides into the ER lumen ${ }^{[28]}$. Besides the $\mathrm{CCP}$ and $\mathrm{iCP}$, mixed proteasomes bearing the subunit composition $\beta 1 \mathrm{c}, \beta 2 \mathrm{c}$ and $\beta 5 \mathrm{i}$ or $\beta 1 \mathrm{c}, \beta 2 \mathrm{i}$ and $\beta 5 \mathrm{i}$ were reported to account for $30-50 \%$ of all cellular $\mathrm{CPs}^{[29]}$ and even CPs with asymmetric composition of $\beta$ subunits were described ${ }^{[30]}$.

The third type of $\mathrm{CP}$, the vertebrate-specific thymoproteasome (tCP), is exclusively expressed in cortical thymic epithelial cells (cTECs) and incorporates the $\mathrm{i}$ subunits $\beta 1 \mathrm{i}$ and $\beta 2 \mathrm{i}$ as well as the exceptional subunit $\beta 5 \mathrm{t}(P S M B 11)^{[21 \mathrm{c}]}$ (Figure 2A). The unique expression profile and subunit composition of the tCP has been implicated to play a pivotal role for the development of $\mathrm{CD}^{+}$cytotoxic $\mathrm{T}$ cells as part of the adaptive immune system.

\subsubsection{Proteolysis by 20S proteasomes}

Structural studies along with mutagenesis experiments proved that a sophisticated hydrogen bond network involving the amino acids Thr1, Asp17, Lys33, Ser129, Asp166 and Ser169 is responsible for the nucleophilicity of the $\gamma$-hydroxylgroup of the active site Thr1 and thus, for proteolytic activity ${ }^{[12-13]}$. A nucleophilic water molecule cluster in the active site induces the proton transfer from $\mathrm{Thr}^{\gamma} \mathrm{O}^{\gamma}$ to $\mathrm{Thr} 1 \mathrm{~N}$, which functions as proton acceptor. Peptide bond hydrolysis then starts with an attack of the $\mathrm{N}$-terminal $\mathrm{Thr}^{\gamma} \mathrm{O}^{\gamma}$ onto the electrophilic carbonyl carbon atom of the scissile peptide bond of substrate proteins (Figure 3). This reaction step creates an acyl-enzyme intermediate and frees the $\mathrm{N}$-terminus of the $\mathrm{C}$-terminal peptide 
fragment. Addition of a pre-oriented nucleophilic water molecule restores the catalytic Thr1 and deliberates the C-terminus of the second cleavage product (Figure 3$)^{[31]}$.

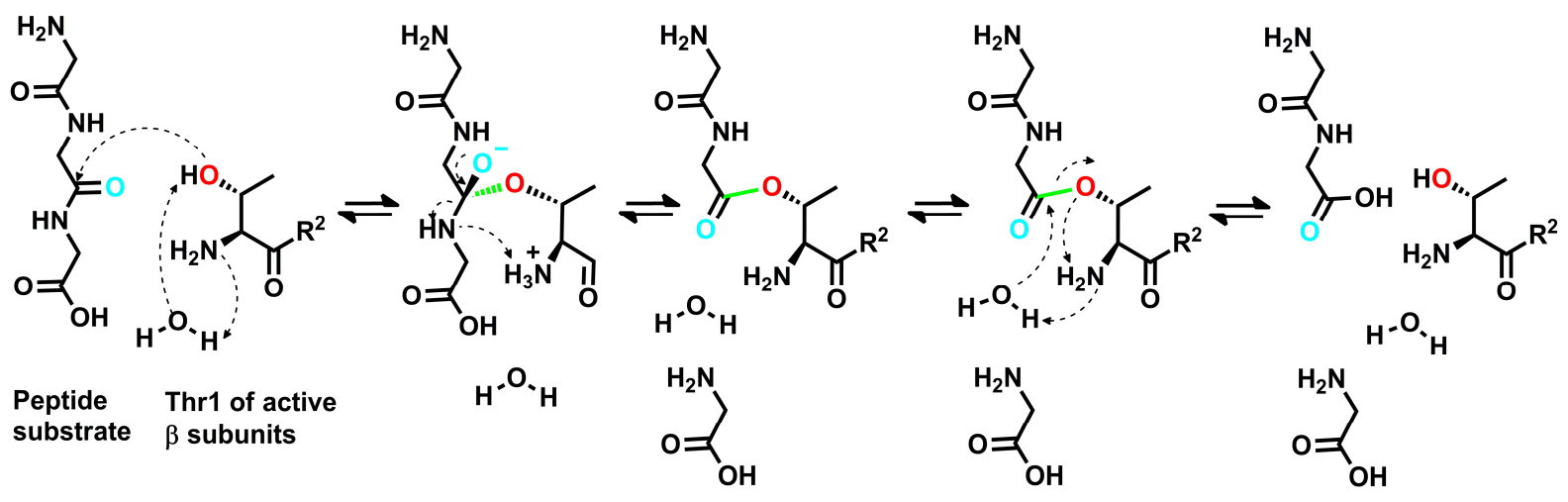

Figure 3 Peptide bond hydrolysis by the proteasome. The nucleophilic $\mathrm{Thr}_{1} \mathrm{O}^{\gamma}$ (red) attacks the electrophilic carbonyl carbon atom of the peptide bond thereby releasing the first cleavage product. In a second step hydrolysis of the formed acyl-enzyme complex (green bond) frees the N-terminal cleavage product and restores the catalytic Thr1.

The proteasome is classified as an endoprotease, as it harbours primed ( $\mathrm{S}^{\prime}$ ', S2', S3') and unprimed substrate binding pockets $(\mathrm{S} 1, \mathrm{~S} 2, \mathrm{~S} 3)$ that are formed by two adjacent subunits ${ }^{[12]}$. The chemical nature of these sites, which accommodate the side chains (P residues) of client proteins (Figure 4), determines the mean residence time of ligands and thus, the cleavage preferences.

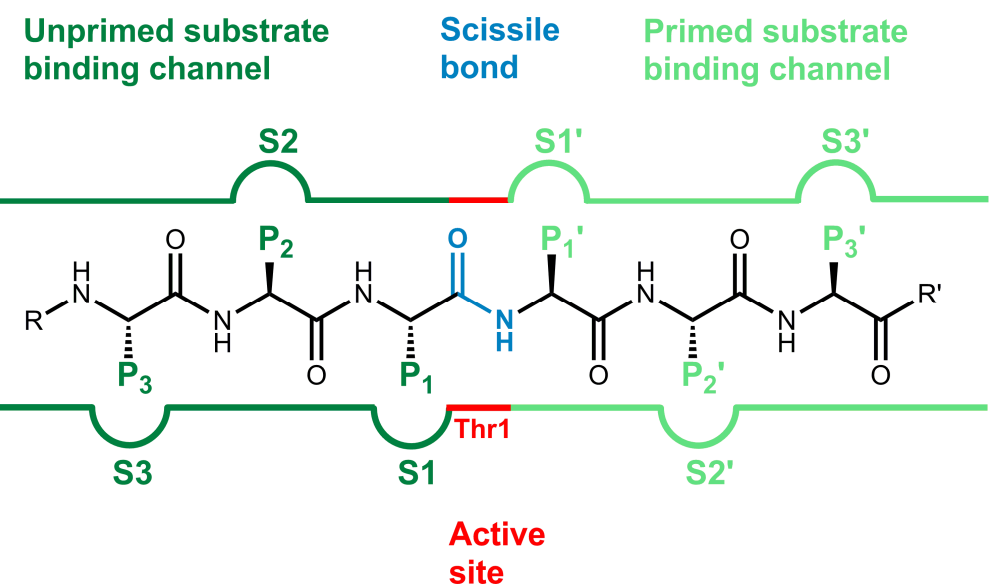

Figure 4 Schematic representation of the substrate binding channel of the proteasomal active sites. The primed pockets ( $\mathrm{S}^{\prime}$; green) and unprimed specificity sites ( $\mathrm{S}$; dark green) bind the ligand's side chains ( $\mathrm{P}^{\prime}$ and $\mathrm{P}$ sites). Afterwards the active site Thr1 (red) cleaves the scissile peptide bond (blue).

Adapted from Huber et al., 2012 ${ }^{[22,32]}$.

In particular the chemical properties of amino acid 45, forming the bottom of the $\mathrm{S} 1$ pocket, decide on proteasomal substrate specificities. The positively charged Arg45 of subunit $y \beta 1 / \beta 1 c$ favours a caspase-like (CL) activity by accommodating acidic residues in the S1 pocket. However, subunit $\beta 1$ can also cleave after some hydrophobic amino acids and thereby 
contributes to the branched chain amino acid preferring (Braap) activity of the proteasome ${ }^{[33]}$. Even though a trypsin-like (TL) activity has been attributed to subunit $y \beta 2 / \beta 2 c$, it can virtually processes after all kind of residues owing to Gly45. The chymotrypsin-like (ChTL) activity, residing in subunit $y \beta 5 / \beta 5 \mathrm{c}$, preferentially hydrolyses proteins C-terminally of hydrophobic amino acids, because its substrate binding channel is lined with apolar residues such as Met45 ${ }^{[13,33 b]}$. In addition, a so-called small neutral amino acid preferring activity (Snaap) has been assigned to the proteasome. For the iCP, previous studies suggested a reduced CL activity, but an enhanced ChTL activity as compared with the $\mathrm{cCP} / \mathrm{yCP}^{[13,33 a, 34]}$. In contrast, the vertebrate-specific tCP has been demonstrated to exert only minimal ChTL $\operatorname{activity}^{[21 c]}$.

\subsubsection{Immune functions of $20 \mathrm{~S}$ proteasomes}

CPs shape the pool of antigenic peptides that are presented by MHC I receptors on the cell surface to immune cells. Importantly, all three types of $\mathrm{CPs}$, the $\mathrm{CCP}$, iCP and tCP, are capable of generating antigens ${ }^{[35]}$. However, their distinct substrate specificities result in different epitope repertoires and physiological implications. Although certain cCP-dependent peptides can trigger immune reactions, the iCP is particularly decisive for this process. Cytokines released during viral infections induce the expression of the iCP in order to enhance antigen presentation and to support clearance of the pathogen. In agreement, mice lacking the iCP bear a significantly altered repertoire of antigens in terms of quantity and quality, implicating that the i subunits enlarge both the abundance and the diversity of MHC I epitopes $^{[36]}$. Besides, the iCP plays a crucial role in $\mathrm{T}$ cell differentiation and the release of proinflammatory cytokines such as interleukin (IL)-23, IL-2 and IFN- $\gamma$ via a yet unkown nuclear factor- $\kappa \mathrm{B}(\mathrm{NF}-\kappa \mathrm{B})-$ independent pathway ${ }^{[37]}$. In addition, proteins that are damaged by cytokine-induced oxidative stress were shown to be efficiently cleared by the $\mathrm{iCP}^{[38]}$.

In contrast to the $\mathrm{cCP}$, the expression of the $\mathrm{tCP}$ is restricted to the cTECs of the thymus ${ }^{[35 \mathrm{~b}]}$. This specialized organ of the immune system selects $\mathrm{T}$ lymphocytes for the optimal interaction strength of their $\mathrm{T}$ cell receptor (TCR) with MHC:self-peptide complexes. Immature thymocytes whose TCR tightly binds to MHC receptors loaded with self-antigens are auto-reactive and are eliminated by negative selection, while $\mathrm{T}$ cells with weak TCR:MHC interactions are considered as self-tolerant and thus survive (positive selection) ${ }^{[23,}$ 39]. The markedly attenuated ChTL activity of the tCP-specific subunit $\beta 5 \mathrm{t}$ is assumed to produce low-affinity self-epitopes for the presentation on MHC receptors ${ }^{[35 b]}$. Moreover, the 
peptide pool created by tCPs in cTECs is unique throughout the body and thus, might prevent autoimmunity, as positively selected $\mathrm{T}$ cells more seldom cross-react with self-peptides that have been generated by the $\mathrm{CCP}$ and iCP outside the cTECs ${ }^{[40]}$. Remarkably, in $\beta 5$ t-depleted mice the number of CTLs is reduced by $75 \%$, stressing the importance of the tCP for the maturation of thymocytes ${ }^{[21 \mathrm{c}]}$. In conclusion, the tCP helps to establish a tolerant $\mathrm{T}$ cell repertoire, the prerequisite for a functional adaptive immune system and the iCP promotes immune surveillance and the elimination of pathogens. Hence, both the tCP and the iCP are key players of the adaptive immune system in vertebrates and their malfunctioning positively correlates with the onset of diverse diseases.

\subsection{S proteasomes: validated and emerging drug targets}

20S proteasomes primarily serve to degrade regulatory or aberrant proteins, but during evolution this function has been exploited for the development of the vertebrate immune system. Thus, the multifaceted functions of CPs make them attractive drug targets for diseases as diverse as cancers and autoimmune disorders.

The CP levels of tumour cells are often upregulated, because their accelerated cell cycle and metabolism require increased turnover rates of proteins. This dependence on $\mathrm{CP}$ activity renders neoplastic cells highly susceptible to proteasome inhibition. Among cancers multiple myeloma cells are most sensitive to $\mathrm{CP}$ inhibitors ${ }^{[41]}$. Myeloma cells are derived from plasma cells and their excessive synthesis of immunoglobulins as well as their chromosomal instability leads to many aberrant and misfolded proteins that have to be removed by CPs. Hence, inhibition of the $\mathrm{CP}$ causes the accumulation of protein aggregates and finally triggers ER stress as well as the unfolded protein response ${ }^{[42]}$. Furthermore, CP inhibitors block proinflammatory signalling cascades such as $\mathrm{NF}-\mathrm{\kappa B}$ and the expression of anti-apoptotic target genes ${ }^{[41 b]}$. Ultimately, tumour suppressor genes like the cyclin kinase inhibitor p2 $7^{\text {kip1[43] }}$ induce apoptosis in transformed cells, while healthy cells remain unaffected ${ }^{[41 \mathrm{a}]}$. This difference in susceptibility creates a therapeutic window for proteasome inhibition in haematological cancers.

However, in certain malignancies, including lung, colon and prostate cancers as well as feline primary fibrosarcoma, tumour pathogenesis depends on elevated iCP and cytokine levels ${ }^{[44]}$. Additionally, Alzheimer's ${ }^{[45]}$ and Huntington's ${ }^{[46]}$ disease as well as amyotrophic lateral sclerosis $^{[47]}$ and inflammatory bowel disease ${ }^{[48]}$ are characterized by increased expression rates of iCPs or single i subunits. Moreover, abnormal levels of i subunits were observed in 
Sjogren's syndrome ${ }^{[49]}$, inclusion body myositis, myofibrillar myopathy ${ }^{[50]}$, Crohn's disease $^{[51]}$ and dextran sulfate sodium-induced colitis ${ }^{[52]}$. These findings suggest a therapeutic benefit from blocking solely the iCP, at least for the above mentioned diseases. So far, inhibition of the $\mathrm{iCP}$ has proven its effectiveness in a number of autoimmune disorders ${ }^{[37,53]}$, but ambiguous results were obtained with respect to its anti-cancer activity. Although a few malignancies were reported to be sensitive to inhibition of the $\mathrm{iCP}^{[44 \mathrm{~b}, 54]}$, other studies demonstrate that only simultaneous blockage of several active sites, for instance of $\beta 5 \mathrm{c}$ and $\beta 5 i$, efficiently causes cell death ${ }^{[55]}$.

In contrast to the $\mathrm{cCP}$ and $\mathrm{iCP}$, the therapeutic potential of $\mathrm{tCP}$ blockage has not been investigated up to now.

\subsection{Types of proteasome inhibitors}

Drug discovery in the ubiquitin-proteasome system has been considerably facilitated by the crystallographic analysis of the CP from $T$. acidophilum $^{[12]}, S$. cerevisiae $^{[13]}$ and Bos taurus $^{[21 \mathrm{a}]}$. Most of the proteasome inhibitors known today harbour an electrophilic head group that reversibly or irreversibly inhibits the catalytic $\mathrm{Thr}_{10}{ }^{\gamma}$. According to their type of pharmacophore covalently acting compounds can be subdivided into seven classes (Figure 5): aldehydes, vinyl sulfones, vinyl amides (syrbactins), boronic acids, $\alpha$ ', $\beta$ 'epoxyketones, $\alpha$ ketoaldehydes (glyoxals) and $\beta$ lactones. Aldehydes, such as the calpain inhibitors I (AcLeu-Leu-norleucinal) and II (Ac-Leu-Leu-methional) were the first inhibitors identified for the proteasome ${ }^{[12,56]}$. However, their susceptibility to oxidation and their off-target activity towards serine and cysteine proteases restrict their medical potential. Vinyl sulfones ${ }^{[57]}$ and the naturally occurring syrbactins ${ }^{[58]}$ form an ether bond with $\mathrm{Thr} 1 \mathrm{O}^{\gamma}$ in a Michael-type 1,4addition, but also target cysteine proteases. Furthermore, $\beta$ lactones are, depending on their $\mathrm{R}^{1}$ substituent, either reversible or irreversible inhibitors of the $\mathrm{CP}^{[59]}$ and its most prominent representative marizomib (salinosporamide A; NPI-0052; Nereus Pharmaceuticals, Inc.) is currently in clinical phase I trials for myeloma, lymphoma and leukaemia ${ }^{[60]}$. Despite their high reactivity and the associated adverse effects, boronic acids such as bortezomib are among the most potent inhibitors of the CP (see also section 3.5). The recently developed second-generation drugs for the proteasome are $\alpha^{\prime}, \beta$ 'epoxyketone-based compounds. Their pharmacophore is derived from the natural product epoxomicin that irreversibly inhibits the $\mathrm{CP}$ in a bivalent reaction involving both $\mathrm{Thr}_{10} \mathrm{O}^{\gamma}$ and $\mathrm{Thr} 1 \mathrm{~N}$ (see 6.3.4) ${ }^{[61]}$. Due to this mode of action $\alpha^{\prime}, \beta^{\prime}$ epoxyketones are highly selective for Ntn hydrolases such as the $20 \mathrm{~S}$ proteasome. 
Employing a reaction mechanism similar to $\alpha^{\prime}, \beta$ 'epoxyketones, $\alpha$ ketoaldehydes also selectively target CPs but their formation of a cyclic Schiffbase with $\mathrm{Thr}^{\gamma} \mathrm{O}^{\gamma}$ and $\mathrm{Thr} 1 \mathrm{~N}$ is reversible $\mathrm{e}^{[62]}$.

Except for lactones and syrbactins, CP ligands consist of a peptidic backbone of two to four amino acids that is attached to one of the listed electrophilic head groups. These peptide-based compounds were shown to mimic natural protein substrates by forming an antiparallel $\beta$ sheet in the substrate binding channels of the $\mathrm{yCP}^{[63]}$.

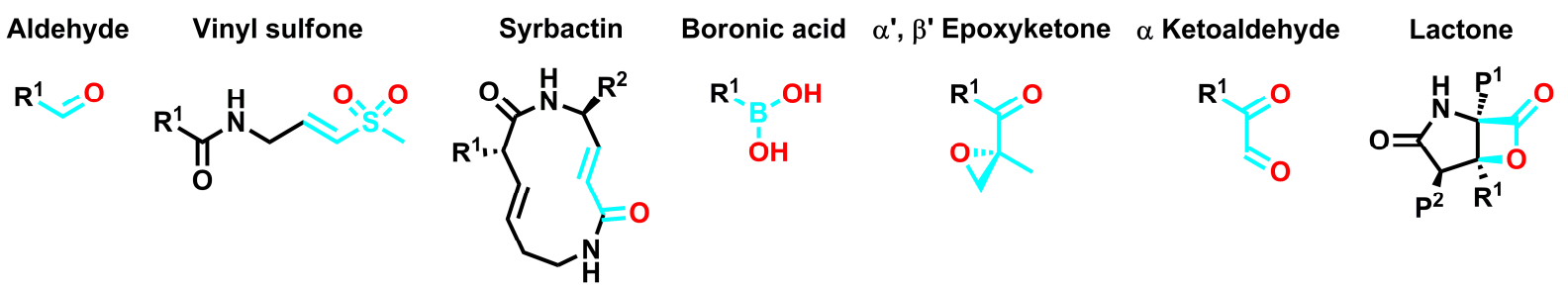

Figure 5 Electrophilic headgroups of covalently acting proteasome inhibitors. The functional groups are shown in cyan with their oxygens marked in red. $\mathrm{R}^{1}$ and $\mathrm{R}^{2}$ designate the variable parts of the compounds. For lactones the $\mathrm{P}^{1}$ and $\mathrm{P}^{2}$ side chains, targeting the corresponding $\mathrm{S}$ pockets of CPs, are depicted.

\subsection{Clinically relevant proteasome inhibitors}

After years of academic and pharmaceutical research, in 2003, bortezomib (Velcade ${ }^{\circledR}$, Millenium Pharmaceuticals, Inc.; PS-341) has been granted full approval by the U. S. food and drug administration (FDA) for the treatment of multiple myeloma as well as relapsed or refractory mantle cell lymphoma (Figure 6) ${ }^{[64]}$. Furthermore, the therapeutic benefit of bortezomib for organ transplantation ${ }^{[65]}$ and solid tumours like non-small cell lung cancer ${ }^{[66]}$ is currently being investigated. With annual sale rates of more than 2 billion dollars bortezomib is a blockbuster drug that substantially elongates the life spans and survival rates of multiple myeloma patients. The dipeptide boronic acid inhibitor potently inhibits the $\beta 5 \mathrm{c}$, $\beta 5 \mathrm{i}$ and $\beta 1 \mathrm{i}$ active sites of the $\mathrm{cCP}$ and $\mathrm{iCP}$ with $\mathrm{IC}_{50}$ values of 3-8 $\mathrm{nM}$ and only in higher concentrations also targets the $\beta 1 \mathrm{c}, \beta 2 \mathrm{c}$ and $\beta 2 \mathrm{i}$ subunits ${ }^{[42 e]}$. Boronic acids such as bortezomib form a reversible tetrahedral transition state with $\mathrm{Thr}^{\gamma} \mathrm{O}^{\gamma}$ that is stabilized by hydrogen bonds with Thr1N and the oxyanion hole Gly47NH of active proteasome subunits ${ }^{[67]}$. Although these interactions promote a higher affinity of bortezomib for Ntn hydrolases, bortezomib was shown to also considerably inhibit serine proteases, including cathepsin G, cathepsin A, chymase, dipeptidyl peptidase II and HtrA2/omi, involved in neuronal survival ${ }^{[68]}$. These off-target activities cause severe neurotoxicity leading to tremor, reduced nerve conduction velocity and nerve degeneration, which affect about $30 \%$ of all 
patients treated with bortezomib ${ }^{[68]}$. Additional drawbacks of bortezomib include thrombocytopenia, neutropenia and gastrointestinal disorders as well as its poor bioavailability that necessitates intravenous administration ${ }^{[69]}$.

Moreover, a significant fraction of newly diagnosed patients does not respond to bortezomib and treated patients often relapse ${ }^{[70]}$. Whereas increased concentrations of the heat shock protein (Hsp) 27 were reported to confer primary resistance ${ }^{[71]}$, the molecular basis for acquired non-responsiveness to bortezomib is still in focus of scientific research efforts. So far, cell culture studies analysing the long-term effects of bortezomib treatment revealed several adaptive mutations in the $\beta 5$ substrate binding channel that convey drug resistance. For instance, the mutations M45V, M45I, A49T, A49V, A50V, C52F and C63F are suggested to impair both the catalytic activity of subunit $\beta 5$ and its affinity for bortezomib, hereby leading to the observed reduction in therapeutic efficacy ${ }^{[72]}$. These findings might also provide an explanation for the up-regulation of subunit $\beta 5$ as a compensation for its decreased activity $^{[72 \mathrm{a}]}$. However, evidence for the clinical relevance of these in vitro identified mutations and their biological impact still has to be adduced. Despite its overwhelming success in the first decade of its application, bortezomib's disadvantages encouraged the development of a second generation of $\mathrm{CP}$ inhibitors.

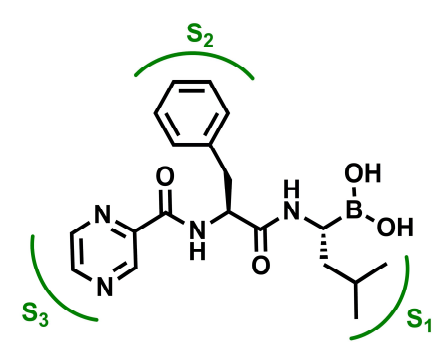

bortezomib (Velcade ${ }^{\circledR}$ )

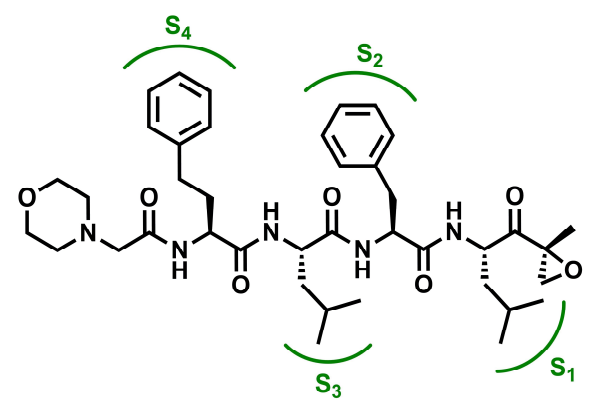

carfilzomib (Kyprolis ${ }^{\mathrm{TM}}$ )

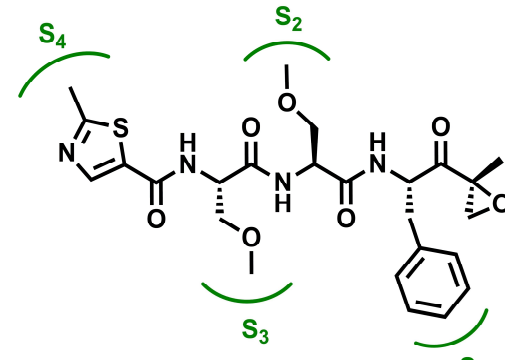

oprozomib (ONX 0912)

Figure 6 Prominent proteasome inhibitors. The chemical structures of the dipeptide boronic acid inhibitor bortezomib, the tetrapeptidic $\alpha$ ', $\beta$ 'epoxyketone carfilzomib and the tripeptide oprozomib are shown. All three compounds target the $\beta 5 \mathrm{c}$ and $\beta 5 \mathrm{i}$ active sites of the proteasome. Bortezomib and carfilzomib are FDA approved for the treatment of multiple myeloma; oprozomib is in phase I clinical trials. The unprimed substrate specificity (S) pockets of the $\mathrm{CP}$ that are targeted by these compounds are indicated in green.

Just recently, in July 2012, one of these novel compounds, carfilzomib (Kyprolis ${ }^{\circledR}$; Onyx Pharmaceuticals, Inc.; PR-171) has been approved by the FDA as a second-line drug for the treatment of patients that relapsed from bortezomib (Figure 6) ${ }^{[73]}$. Carfilzomib belongs to the class of $\alpha^{\prime}, \beta$ 'epoxyketones derived from the microbial natural product epoxomicin. To date, due to their bivalent irreversible reaction mode, $\alpha^{\prime}, \beta$ 'epoxyketones are the most specific 
inhibitors known for the $\mathrm{CP}^{[61]}$ (see 6.3.4). Similarly to bortezomib, carfilzomib potently blocks the $\beta 5 \mathrm{c}$ and $\beta 5 \mathrm{i}$ active sites of $\mathrm{cCP}$ and $\mathrm{iCP}$ with $\mathrm{IC}_{50}$ values of $6 \mathrm{nM}$ and $33 \mathrm{nM}$, respectively, but unlike bortezomib, carfilzomib does not affect $\beta 1 \mathrm{c}^{[74]}$. Although carfilzomib also unintentionally induces neutropenia and thrombocytopenia, it does not cause peripheral neurotoxicity, as observed for the boronic acid bortezomib ${ }^{[68,75]}$.

Oprozomib (Onyx Pharmaceuticals, Inc.; ONX 0912; PR-047), another epoxomicin derivative, is an orally available inhibitor of both the $\beta 5 \mathrm{c}$ and the $\beta 5 \mathrm{i}$ active sites $\left(\mathrm{IC}_{50}(\beta 5 \mathrm{c})\right.$ $36 \mathrm{nM}$; $\left.\mathrm{IC}_{50}(\beta 5 \mathrm{i}) 82 \mathrm{nM}\right)^{[76]}$. Owing to its high cytotoxicity and its superiority to bortezomib and carfilzomib with respect to its mode of administration, oprozomib is currently being explored in Phase I clinical trials as a monotherapy for solid tumours and haematological malignancies.

\subsection{Subunit-specific proteasome inhibitors and their therapeutic potential}

Subunit-specific inhibitory compounds are valuable tools for examining the impact of individual proteasome subunits on cell division and biological signalling pathways. However, the development of compounds with subunit specificities is often hindered by the strong inhibitory potency of most reactive functional head groups (see chapter 3.4). Hence, only ligands that undergo optimal enthalpic interactions with the surrounding protein residues exert pronounced subunit selectivity and for their design structural data are strongly demanded.

A long time drug design efforts solely concentrated on the ChTL activities of the proteasome, but current studies also take into account the CL and TL active sites. Recently, a series of $\alpha$ ', $\beta$ ' epoxyketone inhibitors that target the TL activities of the $\mathrm{CCP}$ and $\mathrm{iCP}$ was published ${ }^{[77]}$. These compounds were demonstrated to render malignant cells more susceptible to bortezomib and carfilzomib than $\beta 1$-selective inhibitors ${ }^{[77]}$. Nonetheless, compounds with selectivity for either $\beta 2 \mathrm{c}$ or $\beta 2 \mathrm{i}$ are still not available. For subunit $\beta 1 \mathrm{i}$ two selective drugs have been reported so far: UK-101, a dihydroeponemycin based $\alpha^{\prime}, \beta$ 'epoxyketone and IPSI-001 (calpeptin), a peptide aldehyde inhibitor. The cytotoxicity of UK-101 towards $\beta 1 \mathrm{i}$ overexpressing prostate cancer cells ${ }^{[44 \mathrm{~b}]}$ underlines the importance of the iCP for the onset and progression of at least certain malignancies. Likewise, anti-tumour activity has been described for IPSI-001 ${ }^{[54 \mathrm{a}]}$, however, its apoptotic effects might result from co-inhibition of $\beta 5$ active sites. For the $\beta 5$ subunits several highly potent and selective ligands are known. The most potent compound is the $\beta 5$ i-targeting $\alpha^{\prime}, \beta$ 'epoxyketone ONX 0914 (PR-957; OnyxPharmaceuticals, Inc.; Figure 7) ${ }^{[37]}$. Remarkably, ONX 0914 showed therapeutic effects 
A

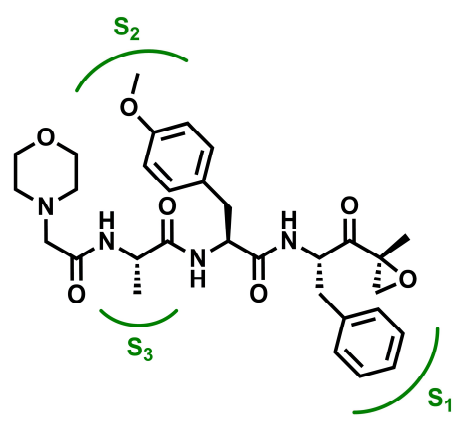

ONX 0914 (PR-957)

B

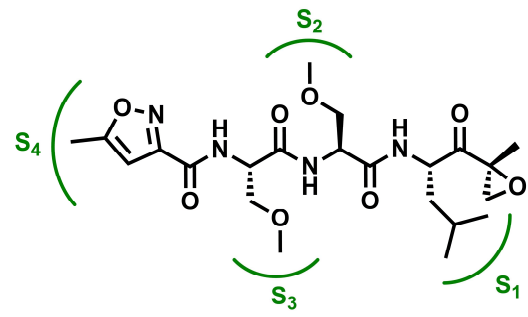

PR-825

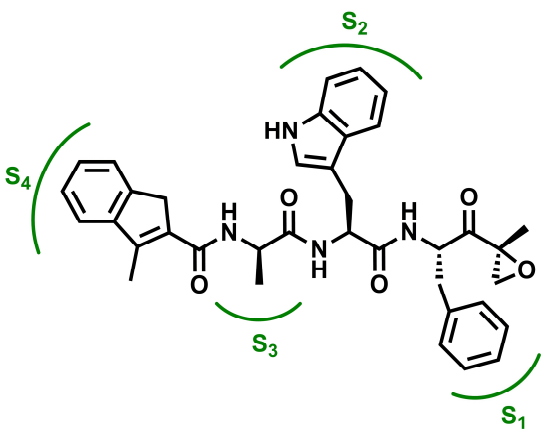

PR-924

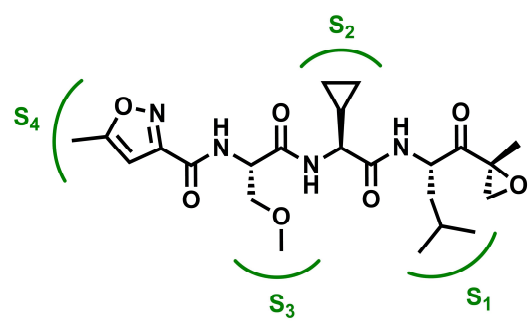

PR-893

Figure 7 Chemical structures of $\beta 5 \mathrm{i}$ - and $\beta 5 \mathrm{c}$-selective compounds. (A) ONX 0914 and PR-924 specifically target subunit $\beta 5$ i of the iCP. (B) PR-825 and PR-893 are selective inhibitors of the $\beta 5 \mathrm{c}$ active site of the cCP. The unprimed substrate specificity pockets of the proteasome that accommodate the side chains of the peptidic inhibitors are indicated in green.

in mouse models of rheumatoid arthritis ${ }^{[37]}$, experimental colitis ${ }^{[53 \mathrm{a}]}$, lupus erythematosus ${ }^{[53 \mathrm{~b}]}$ and Hashimoto's thyroiditis ${ }^{[53 c]}$. These medicinal benefits are based on the reduction of the levels of proinflammatory cytokines such as IL-6, IL-23 and $\mathrm{TNF}^{[37]}$ and the modulation of CTL responses. More specifically, ONX 0914 harms the differentiation and proliferation of $\mathrm{T}_{\mathrm{H}} 1$ and $\mathrm{T}_{\mathrm{H}} 17$ cells but not of regulatory $\mathrm{T}$ lymphocytes ${ }^{[78]}$. In addition, administration of ONX 0914 depresses the expression of MHC I receptors to $50 \%$ and abolishes the production of $\beta 5$ i-restricted antigens. Compared to etanercept, a scavenger of the proinflammatory cytokine TNF- $\alpha$ currently used as an immunosuppressant, ONX 0914 exerts higher potency ${ }^{[37]}$. Based on its promising pharmacological properties and effects in model systems, ONX 0914 is further investigated in preclinical studies for autoimmune diseases. The medicinal potential of the second $\beta 5$ i-selective compound PR-924 (Onyx Pharmaceuticals, Inc.), a structural analogue of ONX 0914, has not yet been evaluated in immunological disorders, but conflicting data have been described with respect to its anti-myeloma activity $^{[54 b, 55]}$. Notably, the two epoxomicin derivatives PR-825 and PR-893 (Onyx Pharmaceuticals, Inc.), that selectively target subunit $\beta 5 \mathrm{c}$, were attributed neither antiinflammatory nor anti-cancer activity ${ }^{[37,55]}$. Hence, cCP and tCP selective drugs remain to be examined for any medicinal use. 


\section{Objective}

Structural information on the yCP alone and in complex with ligands strongly supported the development of the currently known potent $\mathrm{CP}$ inhibitors bortezomib and carfilzomib. Both compounds do not discriminate between the three mammalian $\mathrm{CP}$ types and, thereby, exert anti-cancer activity. However, recent studies demonstrate that iCP-specific inhibitors might qualify as therapeutics in autoimmune diseases. So far, only a few iCP-specific inhibitors were identified, mostly because structural data on the iCP were lacking.

The aim of this thesis was to elucidate the atomic structures of the murine $\mathrm{iCP}$ and $\mathrm{cCP}$ by $\mathrm{X}$ ray crystallography. The crystal structures of both CP types from one organism enable the direct comparison of the structural features of the iCP and the cCP from Mus musculus, the cCP from B. taurus and the $\mathrm{CP}$ of $S$. cerevisiae. The structural data were expected to explain observed differences in the substrate specificities of the iCP and cCP. Moreover the structural characterization aimed at providing insights into the generation of the distinct MHC I peptide patterns by the $\mathrm{CCP}$ and $\mathrm{iCP}$ and into the pivotal role of the $\mathrm{iCP}$ in the adaptive immune system of vertebrates. Besides the examination of the cleavage preferences, it was intended to elucidate the molecular basis for the (non-) selectivity of known CP inhibitors. In particular, cCP and iCP structures in complex with the iCP-specific epoxyketone inhibitor ONX 0914 were supposed to unravel the reason for its $\beta 5$ i selectivity.

Amino acid substitutions in the substrate binding channel are known to affect cleavage and inhibitor specificities. To evaluate the impact of amino acid differences between $\beta 5$ subunits on the proteolytic activity mutagenesis experiments with the model organism $S$. cerevisiae were envisioned. These aimed at imitating the active site surroundings of subunit $\beta 5 \mathrm{c}, \beta 5 \mathrm{i}$ and $\beta 5$ t. Structural data on these mutant yCPs and analysis of their affinity towards ONX 0914 and bortezomib were expected to reveal the effect of the amino acid substitutions on substrate and inhibitor specificities.

In summary, a multidisciplinary approach combining X-ray crystallography, yeast mutagenesis and inhibition assays was intended to provide detailed information on the architecture of all active sites of murine CP types for the development of potential lead structures for diverse medicinal applications. 


\section{Materials and Methods}

\subsection{Materials}

\subsubsection{Chemicals}

All chemicals were obtained from the following companies:

AppliChem (Darmstadt, DE), Biomol (Hamburg, DE), Fluka (Neu-Ulm, DE), Merck (Darmstadt, DE), Sigma-Aldrich (Steinheim, DE), Serva (Heidelberg, DE), Roth (Karlsruhe, DE) and VWR (Darmstadt, DE).

\subsubsection{Antibiotics}

Ampicillin was used in a final concentration of $180 \mathrm{mg} / \mathrm{l}$ for the selection of transformed Escherichia coli (E. coli).

\subsubsection{Media}

$\underline{\mathrm{LB}_{0}} \underline{\text { medium }}$

$\underline{\text { SOC medium }}$

$\underline{\text { YPD medium }}$
Peptone
Yeast extract
$\mathrm{NaCl}$

(Agar

$1 \%(\mathrm{w} / \mathrm{v})$

$0.5 \%(\mathrm{w} / \mathrm{v})$

$0.5 \%(\mathrm{w} / \mathrm{v})$

$2 \%(\mathrm{w} / \mathrm{v}))$

$\begin{array}{ll}\text { Peptone } & 2 \%(\mathrm{w} / \mathrm{v}) \\ \text { Yeast extract } & 0.5 \%(\mathrm{w} / \mathrm{v}) \\ \text { Glucose } & 20 \mathrm{mM} \\ \mathrm{MgSO}_{4} & 10 \mathrm{mM} \\ \mathrm{NaCl} & 10 \mathrm{mM} \\ \mathrm{MgCl}_{2} & 10 \mathrm{mM} \\ \mathrm{KCl} & 2.5 \mathrm{mM}\end{array}$

Yeast extract

$1 \%(\mathrm{w} / \mathrm{v})$

Peptone

$2 \%(\mathrm{w} / \mathrm{v})$

Glucose

$2 \%(\mathrm{w} / \mathrm{v})$

(Agar

$2 \%(\mathrm{w} / \mathrm{v}))$ 


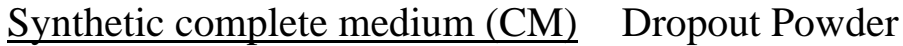

Yeast Nitrogen Base w/o aa

Glucose

pH 5.6

$\underline{\text { CM medium ura }{ }^{-} \text {leu } \text { his }^{-}}$

$\underline{\text { CM medium leu }}{ }^{-}$his $^{-}$5-FOA

\author{
$0.13 \%(\mathrm{w} / \mathrm{v})$ \\ $0.67 \%(\mathrm{w} / \mathrm{v})$ \\ $2 \%(\mathrm{w} / \mathrm{v})$
}

$\begin{array}{ll}\text { 2x CM medium } & 300 \mathrm{ml} \\ 4 \%(w / v) \text { Agar } & 300 \mathrm{ml} \\ 40 \%(w / v) \text { Glucose } & 30 \mathrm{ml} \\ \text { Adenine }(30 \mathrm{mM}) & 6 \mathrm{ml} \\ \text { Tryptophane }(40 \mathrm{mM}) & 6 \mathrm{ml} \\ \text { Lysine }(100 \mathrm{mM}) & 6 \mathrm{ml}\end{array}$

$300 \mathrm{ml}$

$300 \mathrm{ml}$

$30 \mathrm{ml}$

$6 \mathrm{ml}$

$6 \mathrm{ml}$

$6 \mathrm{ml}$

$15 \mathrm{ml}$

$0.6 \mathrm{~g}$

\subsubsection{Enzymes}

Phusion DNA Polymerase (2 U/ $\mu \mathrm{l})$

Pfu Turbo DNA Polymerase ${ }^{\circledR}$

Restriction endonuclease BamHI (20 U/ $\mu \mathrm{l})$

Restriction endonuclease HindIII (20 U/ $\mu \mathrm{l})$

Restriction endonuclease DpnI (20 U/ $\mu \mathrm{l})$

T4 DNA Ligase (1 U/ $\mu \mathrm{l})$

DNAse I
Finnzymes (Vantaa, FI)

Agilent (Santa Clara, US)

New England Biolabs (Ipswich, US)

New England Biolabs (Ipswich, US)

New England Biolabs (Ipswich, US)

Invitrogen (Carlsbad, US)

Sigma-Aldrich (St. Louis, US) 


\subsubsection{Primer}

All oligonucleotides were either HPSF or HPLC purified and dissolved in $\mathrm{ddH}_{2} \mathrm{O}$ to a final concentration of $100 \mathrm{pmol} / \mu \mathrm{l}$. The working concentration of the primer was $10 \mathrm{pmol} / \mu \mathrm{l}$. All primer were synthesized by Eurofins MWG Operon, Ebersberg.

\begin{tabular}{|c|c|}
\hline Primer & Sequence 5' $\rightarrow 3^{\prime}$ \\
\hline $5 / 6 \mathrm{~F}$ & GAAACAGCTATGACCATGAT \\
\hline $5 / 6 \mathrm{R}$ & GACGGCCAGTGAATTGTAAT \\
\hline 20S_22C_for & AGATTCTCGT/GCCACTTGTGGCAATTG \\
\hline 20S_22C_rev & CAATTGCCACAAGTGGA/CACGAGAATCT \\
\hline 27S_31M_rev & CTTCAC/TAGTTTGAGAAGC/AAACCCAATTGC \\
\hline 31M_for & СTTCTCAAACTG/ATGAAGAAAGTTATTG \\
\hline 31M_for2 & СТTCTCAAACTATGAAGAAAGTTATTGAG \\
\hline 31S_for & TCTCAAACTTCTAAGAAAGTTATTG \\
\hline 31S_rev & CAATAACTTTCTTAGAAGTTTGAGA \\
\hline 45T_48T_for & TGGGTACAACTTCCGGTACTGCGGCAG \\
\hline 45T_48T_rev & CTGCCGCAGTACCGGAAGTTGTACCCA \\
\hline 57R_for & TTTTGGGAACGTTGGCTAGGT \\
\hline 57R_rev & ACCTAGCCAACGTTCCCAAAA \\
\hline 32N_for & CTCAAACTG/ATGAATAAAGTTATTG \\
\hline 32N_rev & CAATAACTTTATTCAT/CAGTTTGAG \\
\hline 53S_for & GGCAGATTGTTCATTTTGGG \\
\hline 53S_rev & CCCAAAATGAACAATCTGCC \\
\hline 53S_57R-for & GTTCATTTTGGGAACGTTGGCTAGGTTCT \\
\hline 53S_57R-rev & CAACGTTCCCAAAATGAACAATCTGCCGC \\
\hline SCSTRNG-M45A_for & СATTTTTATTGGGTACCGCGTCTGGTTGTGCGGCAG \\
\hline SCSTRNG-M45A_rev & CTGCCGCACAACCAGACGCGGTACCCAATAAAAATG \\
\hline SCSTRNG-M45V_for & CATTTTTATTGGGTACCGTTTCTGGTTGTGCGGCAG \\
\hline SCSTRNG-M45V_rev & CTGCCGCACAACCAGA $\underline{\overline{\mathrm{AAC}} \text { GGTACCCAATAAAAATG }}$ \\
\hline $71 \mathrm{G} \_$for & CGAGCTGAGGGAAGGTGAACGTATATCGC \\
\hline 71G_rev & GCGATATACGTTCACCTTCCCTCAGCTCG \\
\hline $127 \bar{T}$ for & GACATATTCTGCACTGGTTCAGGTCAA \\
\hline $127 \mathrm{~T} \_r e v$ & TTGACCTGAACCAGTGCAGAATATGTC \\
\hline
\end{tabular}

Table 1 Oligonucleotides used in this work. Underlined nucleotides encode mutations. 


\subsubsection{Strains}

\begin{tabular}{|c|c|c|c|}
\hline Organism & Strain & Genotype & Source \\
\hline E. coli & XL1-Blue & $\begin{array}{l}\text { recAl, endA1, gyrA96, thi-1, } \\
\text { hsdR17, supE44, relA1, lac, } \\
{\left[\mathrm{F}^{\prime}, \text { proAB, lacl }{ }^{\prime} Z \Delta M 15, \text { Tn } 10\right.} \\
\left.\left(\mathrm{Tet}^{\mathrm{r}}\right)\right]\end{array}$ & $\begin{array}{l}\text { Bullock et al., } \\
1987^{[79]}\end{array}$ \\
\hline E. coli & DH5 $\alpha$ & $\begin{array}{l}\text { fhuA2 } \Delta(\arg F-l a c Z) U 169 \text { phoA } \\
\text { glnV44 } \$ 80 \Delta(\text { lacZ }) M 15 \text { gyrA96 } \\
\text { recAl relA1 endA1 thi-1 hsdR17 }\end{array}$ & Hanahan, $1983^{[80]}$ \\
\hline S. cerevisiae & WCG4a & $\begin{array}{l}\text { MATa leu2-3,112 ura3 his3-11,15 } \\
\text { Can }{ }^{\text {GAL2 }}\end{array}$ & $\begin{array}{l}\text { Heinemeyer } \text { et al., } \\
1993^{[81]}\end{array}$ \\
\hline S. cerevisiae & WCG4a pre1-1 & $\begin{array}{l}\text { MATa leu2-3,112 ura3 his } 3-11,15 \\
\text { rad5-535 Can }{ }^{\text {GAL2 }} \text { prel-1 } \\
(\text { S142F) }\end{array}$ & $\begin{array}{l}\text { Heinemeyer } \text { et al. } \\
1991^{[82]}\end{array}$ \\
\hline S. cerevisiae & YWH20a & $\begin{array}{l}\text { MATa leu2-3,112 ura3 his3-11,15 } \\
\text { Can }^{S} \text { GAL2 } \\
\text { pre } 2 \Delta:: H I S 3 \text { [pRS316-E2] }\end{array}$ & $\begin{array}{l}\text { Heinemeyer } \text { et al., } \\
1997^{[83]}\end{array}$ \\
\hline
\end{tabular}

Table $2 E$. coli and yeast strains used in this work.

The E. coli strains XL1-Blue and DH5 $\alpha$ were used for cloning of mutant pre 2 genes in the vector pRS315. The created plasmids were introduced in S. cerevisiae YWH20a. S. cerevisiae WCG4a and WCG4a pre1-1 served as control strains in proteasome activity tests (see section 5.4.1).

\subsubsection{Plasmids}

pRS315

The plasmid pRS315 is a pBluescript-based centromere vector composed of $6 \mathrm{kbp}$. The plasmid encodes the enzyme $\beta$ lactamase, thereby conferring resistance to ampicillin and enabling selection in E. coli. The yeast selectable marker gene is LEU2. Pre2 mutant genes of $1.5 \mathrm{kbp}$ were inserted via the restriction sites BamHI and HindIII into the multiple cloning site. Expression of the pre 2 mutants was controlled by the endogenic PRE2 promoter.

\section{pRS316-E2}

The plasmid pRS316 is pBluescript-based centromere vector composed of $4.9 \mathrm{kbp}$ encoding the ampicillin resistance gene and the URA3 selection marker, suitable for replication in both 
E. coli and yeast. Expression of the wildtype (wt) PRE2 gene, inserted via the restriction sites BamHI and HindIII, was under the control of the endogenic PRE2 promoter.

\begin{tabular}{|c|c|c|c|}
\hline Plasmid & Mutations & Mimic & Source \\
\hline pRS315-PRE2 & - & wt & W. Heinemeyer \\
\hline pRS315-pre2 Q53S & Q53S & $\beta 5 c$ & This work \\
\hline pRS315-pre2 $S$ & A46S & $\beta 5 \mathrm{i}$ & This work \\
\hline pRS315-pre2 C & G48C & $\beta 5 \mathrm{i}$ & This work \\
\hline pRS315-pre2 SC & A46S, G48C & $\beta 5 \mathrm{i}$ & This work \\
\hline pRS315-pre2 MSC & V31M, A46S, G48C & $\beta 5 \mathrm{i}$ & This work \\
\hline pRS315-pre2 SSC & $\mathrm{A} 27 \mathrm{~S}, \mathrm{~A} 46 \mathrm{~S}, \mathrm{G} 48 \mathrm{C}$ & $\beta 5 \mathrm{i}$ & This work \\
\hline pRS315-pre2 SMSC & A27S, V31M, A46S, G48C & $\beta 5 \mathrm{i}$ & This work \\
\hline pRS315-pre2 SCT & $\mathrm{A} 46 \mathrm{~S}, \mathrm{G} 48 \mathrm{C}, \mathrm{V} 127 \mathrm{~T}$ & $\beta 5 \mathrm{i}$ & This work \\
\hline pRS315-pre2 SSCT & A27S, A46S, G48C, V127T & $\beta 5 \mathrm{i}$ & This work \\
\hline pRS315-pre2 MSCT & V31M, A46S, G48C, V127T & $\beta 5 \mathrm{i}$ & This work \\
\hline pRS315-pre2 SMSCT & $\begin{array}{l}\text { A27S, V31M, A46S, G48C, } \\
\text { V127T }\end{array}$ & $\beta 5 \mathrm{i}$ & This work \\
\hline pRS315-pre2 SMSCRT & $\begin{array}{l}\text { A27S, V31M, A46S, G48C, } \\
\text { T57R, V127T }\end{array}$ & $\beta 5 \mathrm{i}$ & This work \\
\hline pRS315-pre2 SNSCRT & $\begin{array}{l}\text { A27S, K32N, A46S, G48C, } \\
\text { T57R, V127T }\end{array}$ & $\beta 5 \mathrm{i}$ & This work \\
\hline pRS315-pre2 SNSCRGT & $\begin{array}{l}\text { A27S, K32N, A46S, G48C, } \\
\text { T57R, K71G, V127T }\end{array}$ & $\beta 5 \mathrm{i}$ & This work \\
\hline pRS315-pre2 SNASCRGT & $\begin{array}{l}\text { A27S, K32N, M45A, A46S, } \\
\text { G48C, T57R, K71G, V127T }\end{array}$ & $\beta 5 \mathrm{i}$ & This work \\
\hline pRS315-pre2 SNVSCRGT & $\begin{array}{l}\text { A27S, K32N, M45V, A46S, } \\
\text { G48C, T57R, K71G, V127T }\end{array}$ & $\beta 5 \mathrm{i}$ & This work \\
\hline pRS315-pre2 $R$ & M45R & $\beta 5 \mathrm{i}$ & W. Heinemeyer ${ }^{[84]}$ \\
\hline pRS315-pre2 TR & $\mathrm{I} 35 \mathrm{~T}, \mathrm{M} 45 \mathrm{R}$ & $\beta 5 \mathrm{i}$ & W. Heinemeyer ${ }^{[84]}$ \\
\hline pRS315-pre2 SCS & A20S, A22C, A46S & $\beta 5 \mathrm{t}$ & This work \\
\hline pRS315-pre2 SS & V31S, A46S & $\beta 5 \mathrm{t}$ & This work \\
\hline pRS315-pre2 SCSS & A20S, A22C, V31S, A46S & $\beta 5 \mathrm{t}$ & This work \\
\hline pRS315-pre2 TST & $\mathrm{M} 45 \mathrm{~T}, \mathrm{~A} 46 \mathrm{~S}, \mathrm{G} 48 \mathrm{~T}$ & $\beta 5 \mathrm{t}$ & This work \\
\hline pRS315-pre2 STST & V31S, M45T, A46S, G48T & $\beta 5 \mathrm{t}$ & This work \\
\hline pRS315-pre2 SCTST & $\begin{array}{l}\text { A20S, A22C, M45T, A46S, } \\
\text { G48T }\end{array}$ & $\beta 5 \mathrm{t}$ & This work \\
\hline pRS315-pre2 SCSTST & $\begin{array}{l}\text { A20S, A22C, V31S, M45T, } \\
\text { A46S, G48T }\end{array}$ & $\beta 5 \mathrm{t}$ & This work \\
\hline
\end{tabular}

Table 3 Plasmids used or created in this work.

\subsubsection{Fluorogenic substrates}

Cbz-Gly-Gly-Leu-pNA

Suc-Leu-Leu-Val-Tyr-AMC

Cbz-Leu-Leu-Glu-AMC
Bachem (Bubendorf, Switzerland)

Bachem (Bubendorf, Switzerland)

Bachem (Bubendorf, Switzerland)

All fluorogenic substrates were dissolved in DMSO and stored at $-20{ }^{\circ} \mathrm{C}$. 


\subsubsection{DNA and protein standards}

peqGOLD DNA Ladder Mix (100 -10 000 bp) Peqlab (Erlangen, DE)

Roti $^{\circledR}$-Mark STANDARD (14-200 kDa) Carl Roth (Karlsruhe, DE)

\subsubsection{Instruments}

\section{Balances}

Analytical Balance TE124S

Sartorius (Göttingen, DE)

Precision Balance BP3100 P

Sartorius (Göttingen, DE)

\section{Centrifuges}

Biofuge Pico

SIGMA 4K15 rotor $11150 / 13220$

rotor $11150 / 13350$

SIGMA 6-16K rotor 12500

Heraeus Instruments (Hanau, DE)

SIGMA Laborzentrifugen

(Osterode am Harz, DE)

SIGMA Laborzentrifugen

(Osterode am Harz, DE)

SIGMA 8K rotor 11805

SIGMA Laborzentrifugen

(Osterode am Harz, DE)

\section{Crystallography}

Art Robbins Instruments Intelli-Plates (96 well)

Dunn Labortechnik (Asbach, DE)

Art Robbins Instruments Phoenix

Dunn Labortechnik (Asbach, DE)

Cooled Incubator Series 3000

CrystalCap $\mathrm{HT}^{\mathrm{TM}}$ für CryoLoop ${ }^{\mathrm{TM}}$

RUMED ${ }^{\circledR}$ Rubarth Apparate (Laatzen, DE)

CrystalCap HT $^{\mathrm{TM}}$ Vial

Hampton (Aliso Viejo, US)

CrystalWand Magnetic ${ }^{\mathrm{TM}}$

Hampton (Aliso Viejo, US)

Hampton (Aliso Viejo, US)

Foam dewar

Spearlab (San Francisco, US)

Magnetic Caps, Pins and Vials

Molecular Dimensions (Newmarket, UK)

MICORLAB ${ }^{\circledR}$ STARlet

Hamilton (Reno, US)

Micro Tool Box

Molecular Dimensions (Newmarket, UK)

Mounted CryoLoop ${ }^{\mathrm{TM}}$

Hampton (Aliso Viejo, US)

Protein Crystallization Screening Suites

QIAGEN (Hilden, DE)

Quick Combi Sealer Plus

HJ-Bioanalytik (Mönchengladbach, DE) 
Siliconized Glass Cover Slides

SuperClear Pregreased 24 Well Plate

Vial clamp

Zoom stereo microscope SZX10/KL1500LCD

\section{Electrophoresis}

Chamber and tray

Digital Graphic Printer UP-D897

Electrophoresis Power Supply EPS 600

Gel documentation system G:BOX

Mini PROTEAN ${ }^{\circledR}$ Cell

PowerPAc Basic Power Supply

\section{Liquid chromatography}

ÄKTAprime ${ }^{\mathrm{TM}}$ plus

ÄKTApurifier ${ }^{\mathrm{TM}}$

$\mathrm{CHT}^{\mathrm{TM}}$ Ceramic Hydroxyapatite

Phenyl Sepharose 6 Fast Flow

RESOURCE $^{\mathrm{TM}} \mathrm{Q}, 6 \mathrm{ml}$

HiPrep $^{\text {TM }}$ 26/10 desalting column

\section{Additional equipments and materials}

Cary Eclipse Fluorescence spectrometer

NanoPhotometer ${ }^{\mathrm{TM}}$ Pearl

Ultraspec10 Cell Density Meter

Constant Cell Disruption System E1061

Electroporation cuvette, $2 \mathrm{~mm}$

Gene Pulser mit Pulse Controller

Incubator

Infors HT Multitron 2 Cell Shaker

inoLab ${ }^{\circledR}$ pH 720 pH-Meter

Laboklav 25/195

MR Hei-Standard Magentic stirrer

Techne Dri-Block DB 2A
Hampton (Aliso Viejo, US)

Crystalgen (New York, US)

Molecular Dimensions (Newmarket, UK)

Olympus (Tokio, JP)

Appligene (Watford, UK)

Sony (Minato, JP)

Pharmacia Biotech (Uppsala, SE)

Syngene (Cambridge, US)

BioRad (Hercules, US)

BioRad (Hercules, US)

GE Healthcare (Chalfont St. Giles, UK)

GE Healthcare (Chalfont St. Giles, UK)

BioRad (Hercules, US)

GE Healthcare (Chalfont St. Giles, UK)

GE Healthcare (Chalfont St. Giles, UK)

GE Healthcare (Chalfont St. Giles, UK)

Varian (Darmstadt, DE)

IMPLEN (München, DE)

Amersham Bioscience (Uppsala, SE)

Constant Systems (Northants, UK)

PeqLab (Erlangen, DE)

BioRad (Hercules, US)

Binder (Tuttlingen, DE)

INFORS HT (Bottmingen/Basel, $\mathrm{CH}$ )

WTW (Weilheim, DE)

SHP Steriltechnik (Magdeburg, DE)

Heidolph (Schwabach, DE)

Bibby Scientific (Stone, UK) 
Thermomixer comfort

Thermocycler MyCycler ${ }^{\mathrm{TM}}$

Vortex Genie 2

White 96 well plate NUNC
Eppendorf (Hamburg, DE)

BioRad (Hercules, US)

Scientific Industries (New York, US)

Thermo scientific (München, DE)

\subsubsection{Computer software and bioinformatics tools}

ApE - A plasmid Editor

BOBSCRIPT

CCP4 Software Suite

ChemDraw

Coot

CorelDRAW X5

DNAman

EndNote X4

GraphPad Prism 5

MAIN

Microsoft Office

MOLSCRIPT

PyMOL Molecular Graphics System

QuikChange ${ }^{\circledR}$ Primer Design Tool

Sybyl

UNICORN $^{\mathrm{TM}}$ control software

XDS Program Package
Wayne Davis, $\mathrm{M}$.

Esnouf, R. M. ${ }^{[85]}$

www.ccp4.ac.uk ${ }^{[86]}$

Perkin Elmer (Cambridge, US)

Emsley, P. ${ }^{[87]}$

Corel (Ottawa, CA)

Lynnon Corporation (Quebec, CA)

Adept Scientific (Frankfurt, DE)

GraphPad Software Inc. (La Jolla, USA)

Turk, D. ${ }^{[88]}$

Microsoft (Redmond, US)

Kraulis P. J. ${ }^{[89]}$

Schrödinger, LLC ${ }^{[90]}$

Agilent Technologies

Tripos (St. Louis, US) ${ }^{[91]}$

GE Healthcare (Chalfont St. Giles, UK)

Kabsch, W. (Heidelberg, DE) ${ }^{[92]}$

DNA and protein sequences were obtained from the "Universal protein resource" (www.uniprot.org). The ProtParam tool (http://www.expasy.ch/tools/protparam.html) was used to calculate physical and chemical parameters such as molecular mass, $\mathrm{pI}$ and the extinction coefficient of protein sequences. Multiple DNA or protein sequences were aligned with the T-Coffee tool (http://www.ebi.ac.uk/Tools/t-coffee/index.html). For the calculation of protein surface areas the online tool PISA (Protein interfaces, surfaces and assemblies service PISA at European Bioinformatics Institute (http://www.ebi.ac.uk/pdbe/prot_int/ pistart.html) ${ }^{[93]}$ ) was used. 


\subsection{Genetic methods}

\subsubsection{Cultivation and long-term storage of Escherichia coli}

E. coli were cultivated either in liquid $\mathrm{LB}_{\mathrm{Amp}}$ medium or on $\mathrm{LB}_{\mathrm{Amp}}$ agar plates. Liquid cultures were inoculated from single colonies and incubated at $37^{\circ} \mathrm{C}$ and $130 \mathrm{rpm}$ in a shaker. Streaks on agar plates were grown overnight at $37{ }^{\circ} \mathrm{C}$ and later on stored at $4{ }^{\circ} \mathrm{C}$ in the fridge. For long-term preservation of E. coli clones glycerol stocks were prepared: an overnight culture was pelleted for $5 \mathrm{~min}$ at $3000 \mathrm{rpm}$, cells were resuspended in $\mathrm{LB}_{0}$ medium containing $30 \%$ (v/v) glycerol, transferred into a cryo tube and frozen in liquid nitrogen.

\subsubsection{Cultivation and long-term storage of Saccharomyces cerevisiae}

Baker's yeast was cultivated at $30{ }^{\circ} \mathrm{C}$ either on agar plates or in liquid medium. Wt and mutant yeast strains were streaked on YPD plates or on CM plates containing the appropriate selection marker composition. Agar plates were stored for 2 to 3 days at $30^{\circ} \mathrm{C}$ and later on kept at $4{ }^{\circ} \mathrm{C}$ in the fridge. For the large scale cultivation of yeast in liquid medium $50-300 \mathrm{ml}$ YPD medium were inoculated with a single colony grown on a YPD agar plate. After incubation for 12-24 h at $130 \mathrm{rpm}$ this preculture was used to inoculate the main culture of 3181 of YPD medium in a ratio of 1:50. The main culture was grown up to $48 \mathrm{~h}$ at $30{ }^{\circ} \mathrm{C}$ and $130 \mathrm{rpm}$ to an $\mathrm{OD}_{600 \mathrm{~nm}}$ of approximately 7 and subsequently harvested for $20 \mathrm{~min}$ at $5000 \mathrm{rpm}$. The cells were washed in $\mathrm{ddH}_{2} \mathrm{O}$, transferred into $50 \mathrm{ml}$ falcons, centrifuged again and finally frozen at $-20{ }^{\circ} \mathrm{C}$. For long-term storage of $S$. cerevisiae clones glycerol stocks were prepared: With a sterile toothpick cells were scraped off from an agar plate, resuspended in $1 \mathrm{ml}$ of $15 \%(\mathrm{v} / \mathrm{v})$ glycerol, transferred into a cryo tube and frozen at $-80{ }^{\circ} \mathrm{C}$.

\subsubsection{Polymerase chain reaction}

The polymerase chain reaction (PCR) served to amplify target genes encoded on genomic DNA or plasmids ${ }^{[94]}$. Annealing temperatures were chosen $5{ }^{\circ} \mathrm{C}$ below the melting temperatures $\left(\mathrm{T}_{\mathrm{m}}\right)$ of the used primers. The $\mathrm{T}_{\mathrm{m}}$ was calculated according to formula 1 or by the QuikChange ${ }^{\circledR}$ Primer Design Tool from Agilent Technologies.

$$
T_{m}=2^{\circ} C \cdot\left(B_{\text {Adenin }}+B_{\text {Thymin }}\right)+4^{\circ} \mathrm{C} \cdot\left(B_{\text {Guanin }}+B_{\text {Cyto sin }}\right)
$$

Formula 1: Calculation of the melting temperature of oligonucleotides. 
In general, PCR reactions were performed according to the following pipetting and temperature schemes:

\begin{tabular}{lll}
\hline Compound & Final concentration & Volume $[\boldsymbol{\mu l}]$ \\
\hline Template & $0.001-0.01 \mathrm{ng} / \mu \mathrm{l}$ & $1-5$ \\
dNTP-Mix $(10 \mathrm{mM})$ & $0.2 \mathrm{mM}$ & 2 \\
Forward Primer $(10 \mathrm{pmol} / \mu \mathrm{l})$ & $0.5 \mu \mathrm{M}$ & 5 \\
Reverse Primer $(10 \mathrm{pmol} / \mu \mathrm{l})$ & $0.5 \mu \mathrm{M}$ & 5 \\
Phusion ${ }^{\circledR} \mathrm{HF}$ buffer $(5 \mathrm{x})$ & $1 \mathrm{x}$ & 20 \\
Phusion ${ }^{\circledR}$ DNA polymerase $(2 \mathrm{U} / \mu \mathrm{l})$ & $0.02 \mathrm{U} / \mu \mathrm{l}$ & 1 \\
$\mathrm{ddH}_{2} \mathrm{O}$ & & Fill up to 100 \\
\hline
\end{tabular}

Table 4 Components of a PCR reaction using the Phusion ${ }^{\circledR}$ DNA polymerase.

$\left.\begin{array}{lll}\hline \text { PCR step } & \text { Temperature } & \text { Time } \\ \hline \text { Initial denaturing } & 95^{\circ} \mathrm{C} & 3 \mathrm{~min} \\ \text { Denaturing } & 95^{\circ} \mathrm{C} & 30 \mathrm{sec} \\ \text { Annealing } & 55^{\circ} \mathrm{C} & 30 \mathrm{sec} \\ \text { Elongation } & 72^{\circ} \mathrm{C} & \text { variable }\end{array}\right\} 35$ cycles

Table 5 Temperature program for PCR reactions using the Phusion ${ }^{\circledR}$ DNA polymerase.

For site-directed mutagenesis alternatively the QuikChange ${ }^{\circledR}$ PCR was used (see also section 5.2.4):

\begin{tabular}{lll}
\hline Compound & Final concentration & Volume $[\boldsymbol{\mu l}]$ \\
\hline Template & $0.2-1 \mathrm{ng} / \mu \mathrm{l}$ & $\mathrm{x}$ \\
dNTP-Mix $(10 \mathrm{mM})$ & $0.2 \mathrm{mM}$ & 1 \\
Forward Primer $(10 \mathrm{pmol} / \mu \mathrm{l})$ & $2.5 \mathrm{ng} / \mu \mathrm{l}$ & $\mathrm{y}$ \\
Reverse Primer $(10 \mathrm{pmol} / \mu \mathrm{l})$ & $2.5 \mathrm{ng} / \mu \mathrm{l}$ & $\mathrm{Z}$ \\
Pfu buffer $(10 \mathrm{x})$ & $1 \mathrm{x}$ & 5 \\
Pfu Turbo DNA polymerase ${ }^{\circledR}$ & & 1 \\
$\mathrm{ddH}_{2} \mathrm{O}$ & & Fill up to 50 \\
\hline
\end{tabular}

Table 6 Components of a PCR reaction using the Pfu Turbo DNA polymerase ${ }^{\circledR}$.

$\left.\begin{array}{lll}\hline \text { PCR step } & \text { Temperature } & \text { Time } \\ \hline \text { Initial denaturing } & 95^{\circ} \mathrm{C} & 30 \mathrm{sec} \\ \text { Denaturing } & 95^{\circ} \mathrm{C} & 30 \mathrm{sec} \\ \text { Annealing } & 55^{\circ} \mathrm{C} & 1 \mathrm{~min} \\ \text { Elongation } & 68^{\circ} \mathrm{C} & 7.5 \mathrm{~min}\end{array}\right\} 16$ cycles

Table 7 Temperature program for QuikChange ${ }^{\circledR} \mathrm{PCR}$ reactions. 


\subsubsection{Site-directed mutagenesis}

Mutations in the yeast proteasome gene PRE2 encoding the subunit y $\beta 5$ were introduced either via recombinant PCR techniques or QuikChange ${ }^{\circledR}$ mutagenesis.

The first method required three PCR reactions and four oligonucleotides two of which encoding the desired mutation:

PCR 1a: Primer 5/6F and Mutation_rev Primer; elongation time $45 \mathrm{~s}$

PCR 1b: Primer 5/6R and Mutation_for Primer; elongation time $45 \mathrm{~s}$

PCR 2: Primer 5/6F and 5/6R with the PCR products $1 \mathrm{a}$ and $1 \mathrm{~b}$ as template; elongation time $90 \mathrm{~s}$.

The PCR product 2 was digested, inserted into the vector pRS315 and subsequently transformed into E. coli.

The QuikChange ${ }^{\circledR}$ mutagenesis ${ }^{[95]}$ required two HPLC purified primers, containing the desired mutation. Usually, different amounts of template DNA were tested. After the PCR reaction $1 \mu \mathrm{l}$ of the restriction endonucleoase $D p n I$ was added and the sample was incubated for $1.5 \mathrm{~h}$ at $37^{\circ} \mathrm{C}$. DpnI selectively degraded only the methylated template DNA. Finally, $3 \mu \mathrm{l}$ DNA were transformed into E. coli and all cells were streaked on an $\mathrm{LB}_{\mathrm{Amp}}$ agar plate.

\subsubsection{Agarose gel electrophoresis}

For analytical and preparative separations of DNA $1 \%(\mathrm{w} / \mathrm{v})$ agarose gels in 1x Tris-AcetateEDTA buffer (TAE) were used. Samples were supplemented with DNA loading dye (final concentration: $1 \mathrm{x}$ ) and loaded on the gel. Electrophoresis was carried out for $40 \mathrm{~min}$ in $1 \mathrm{x}$ TAE buffer at $120 \mathrm{~V}$. Staining of the gel in a solution of $1 \mathrm{mg} / \mathrm{l}$ ethidium bromide for $20 \mathrm{~min}$ enabled visualization of DNA bands under UV light of $365 \mathrm{~nm}$.

DNA loading dye (10x)

$\begin{array}{ll}\text { Tris-HCl, pH } 8.2 & 10 \mathrm{mM} \\ \text { EDTA } & 1 \mathrm{mM} \\ \text { Glycerol } & 50 \%(\mathrm{v} / \mathrm{v}) \\ \text { Xylene cyanole } & 0.25 \%(\mathrm{w} / \mathrm{v}) \\ \text { Bromphenol blue } & 0.25 \%(\mathrm{w} / \mathrm{v})\end{array}$


$\underline{\text { TAE buffer }(50 \mathrm{x})}$

Tris-Ac, $\mathrm{pH} 8.2 \quad 2 \mathrm{M}$

EDTA

$100 \mathrm{mM}$

\subsubsection{Isolation of plasmid DNA from $E$. coli}

Isolation of plasmid DNA from E. coli was carried out with the peqGOLD Plasmid Miniprep

Kit I or II according to the instruction manual. Purified plasmids were stored at $-20{ }^{\circ} \mathrm{C}$.

\subsubsection{Purification of DNA}

DNA was purified by the peqGOLD Gel extraction or the peqGOLD Cycle-Pure Kit according to the manufacturer's instruction. Purified DNA samples were stored at $-20{ }^{\circ} \mathrm{C}$.

\subsubsection{Restriction digest of DNA}

Preparative restriction digests of a total volume of $70 \mu$ l contained $40 \mathrm{U}$ per restriction endonuclease in the recommended buffer and were incubated for 2 to $5 \mathrm{~h}$ at $37^{\circ} \mathrm{C}$. Analytical cleavages were performed in a total volume of $10 \mu \mathrm{l}$ containing the appropriate buffer and $5 \mathrm{U}$ per restriction endonuclease. Analytical digests were stored for $1.5 \mathrm{~h}$ at $37^{\circ} \mathrm{C}$.

\begin{tabular}{lll}
\hline Compound & Final concentration & Volume $[\boldsymbol{\mu l}]$ \\
\hline DNA & & $\mathrm{Up}$ to 52 \\
NEB buffer 2(10x) & $1 \mathrm{x}$ & 7 \\
$\mathrm{BSA}(10 \mathrm{x})$ & $1 \mathrm{x}$ & 7 \\
Bam HI $(20 \mathrm{U} / \mu \mathrm{l})$ & $0.6 \mathrm{U} / \mu \mathrm{l}$ & 2 \\
HindIII $(20 \mathrm{U} / \mu \mathrm{l})$ & $0.6 \mathrm{U} / \mu \mathrm{l}$ & 2 \\
$\mathrm{H}_{2} \mathrm{O}$ & & Add to 70 \\
\hline
\end{tabular}

Table 8 Pipetting scheme for preparative restriction digests.

\begin{tabular}{lll}
\hline Compound & Final concentration & Volume $[\boldsymbol{\mu l}]$ \\
\hline Plasmid & & 2 \\
NEB buffer 2(10x) & $1 \mathrm{x}$ & 1 \\
$\mathrm{BSA}(10 \mathrm{x})$ & $1 \mathrm{x}$ & 1 \\
Bam HI $(20 \mathrm{U} / \mu \mathrm{l})$ & $0.5 \mathrm{U} / \mu \mathrm{l}$ & 0.25 \\
HindIII $(20 \mathrm{U} / \mu \mathrm{l})$ & $0.5 \mathrm{U} / \mu \mathrm{l}$ & 0.25 \\
$\mathrm{H}_{2} \mathrm{O}$ & & 5.5 \\
\hline
\end{tabular}

Table 9 Pipetting scheme for analytical restriction digests. 


\subsubsection{Determination of DNA concentration}

DNA concentrations were determined by measuring the absorption at $260 \mathrm{~nm}$ with the nanophotometer (IMPLEN); an $\mathrm{OD}_{260 \mathrm{~nm}}$ of 1 corresponds to a concentration of $50 \mathrm{ng} / \mu \mathrm{l}$ DNA.

\subsubsection{Ligation of DNA fragments}

Vector and insert amounts were calculated according to formula 2.

$$
m_{\text {insert }}=\frac{m_{\text {vector }} \cdot b p_{\text {insert }}}{b p_{\text {vector }}} \cdot x
$$

Formula 2: Calculation of vector and insert amounts for the ligation of DNA fragments. For standard reactions $\mathrm{m}_{\text {vector }}$ was 50-100 ng and $\mathrm{x}=3-5$.

\begin{tabular}{lll}
\hline Compound & Final concentration & Volume $[\boldsymbol{\mu l}]$ \\
\hline Plasmid & $5-10 \mathrm{ng} / \mu \mathrm{l}$ & $\mathrm{x}$ \\
Insert & & $\mathrm{y}$ \\
$\mathrm{H}_{2} \mathrm{O}$ & & $7.5-(\mathrm{x}+\mathrm{y})$ \\
T4 DNA ligation buffer $(5 \mathrm{x})$ & $1 \mathrm{x}$ & 2 \\
T4 DNA ligase $(1 \mathrm{U} / \mu \mathrm{l})$ & $0.05 \mathrm{U} / \mu \mathrm{l}$ & 0.5 \\
\hline
\end{tabular}

Table 10: Pipetting scheme for the ligation of DNA fragments.

To unwind DNA fragments both plasmid and insert were incubated for $10 \mathrm{~min}$ at $55{ }^{\circ} \mathrm{C}$ and for $5 \mathrm{~min}$ on ice. Afterwards ligation buffer and T4 ligase were added. The sample was either kept at $4{ }^{\circ} \mathrm{C}$ overnight or at room temperature for one hour.

\subsubsection{Transformation of $E$. coli by electroporation}

$40 \mu 1$ of electrocompetent $E$. coli cells and $1 \mu 1$ of plasmid DNA were mixed and transferred into a $2 \mathrm{~mm}$ electroporation cuvette. Transformation of $E$. coli was achieved by applying a high voltage pulse of approximately $5.7 \mathrm{~ms}$ and $2500 \mathrm{~V}^{[96]}$. Subsequently, cells were resuspended in $1 \mathrm{ml}$ of SOC medium and incubated for $1 \mathrm{~h}$ at $37{ }^{\circ} \mathrm{C}$ and $500 \mathrm{rpm}$ shaking to recover. Different dilutions of cells were plated on agar plates containing ampicillin. 


\subsubsection{Transformation of $S$. cerevisiae}

S. cerevisiae was transformed according to the LiAc method by Gietz and Woods ${ }^{[97]}$. Yeast cells were picked with a sterile toothpick and resuspended in $1 \mathrm{ml}$ of sterile water. After a short spin down at $13000 \mathrm{rpm}$ for $30 \mathrm{sec}$ the supernatant was discarded and the pelleted cells were taken up in 1xTE/LiAc buffer. An additional step of centrifugation followed. Then, yeast cells were resuspended in $\mathrm{x}$ times $50 \mu \mathrm{l} 1 \mathrm{x}$ TE/LiAc and $\mathrm{x}$ times $4 \mu \mathrm{l}$ boiled single stranded salmon carrier DNA from salmon testes (Sigma-Aldrich (St. Louis, US)). The resulting cell suspension was split into $\mathrm{x}$ tubes and each was supplemented with $5 \mu \mathrm{l}$ of plasmid DNA to be transformed and $300 \mu \mathrm{l}$ of PEG3350/TE/LiAc (8:1:1). The samples were gently mixed and incubated for $30 \mathrm{~min}$ at $30{ }^{\circ} \mathrm{C}$. After cells have been heated to $42{ }^{\circ} \mathrm{C}$ for $20 \mathrm{~min}$, they were centrifuged for $2 \mathrm{~min}$ at $2000 \mathrm{rpm}$, resuspended in $100 \mu 1$ 1xTE buffer and streaked on $\mathrm{CM}^{-}$ura $^{-}$leu his ${ }^{-}$plates.

$\underline{\text { TE buffer (10x) }}$

$$
\begin{array}{ll}
\text { Tris-HCl, pH 7.4 } & 100 \mathrm{mM} \\
\text { EDTA } & 10 \mathrm{mM}
\end{array}
$$

$\underline{\operatorname{LiAc}(10 x)}$

LiAc, pH 7.5

$1 \mathrm{M}$

$\underline{\operatorname{PEG} 3350(10 x)}$

PEG3350

$50 \%(\mathrm{w} / \mathrm{v})$

\subsubsection{Plasmid shuffling}

Yeast proteasome mutants were created by the plasmid shuffling procedure ${ }^{[98]}$. The haploid yeast strain YWH20a, which is chromosomally deleted in its wildtype PRE2 gene and instead carries a wt gene copy on an URA3 episome (pRS316), was transformed by pre 2 gene variants, encoded on the plasmid pRS315 carrying the LEU2 selection marker (see Table 3).

2 to 3 days after the transformation single colonies were picked, streaked either on an YPD or $\mathrm{CM}$ leu ${ }^{-}$his ${ }^{-}$plate as patches and grown for additional 2 days at $30{ }^{\circ} \mathrm{C}$. To select for clones that have lost the wt PRE2 gene copy, transformants were grown on $\mathrm{CM}$ leu his ${ }^{-}$medium containing 5-FOA. Optionally, after 2 to 3 days of incubation at $30{ }^{\circ} \mathrm{C}$ a second round of 5FOA selection can be made. Finally, single colonies were picked from the 5-FOA containing plates and transferred onto YPD agar. Due to the indispensable need for a functional PRE2 gene for yeast survival no further selection was required. 


\subsubsection{DNA isolation from $S$. cerevisiae}

Yeast cells were scraped off from an agar plate with a toothpick and resuspended in $500 \mu 1$ of $\mathrm{ddH}_{2} \mathrm{O}$. Cells were centrifuged for $30 \mathrm{sec}$ at $14000 \mathrm{rpm}$. The cell pellet was taken up in $200 \mu 1$ of breaking buffer. After the addition of $200 \mu 1$ of glass beads $(0.5 \mathrm{~mm})$ and $200 \mu \mathrm{l}$ of phenol/chloroform/isoamyl alcohol (25:24:1) solution, the samples were vortexed for $2 \mathrm{~min}$. Cell debris was pelleted by centrifugation for $5 \mathrm{~min}$ at $140000 \mathrm{rpm}$. $100 \mu \mathrm{l}$ supernatant were mixed with $10 \mu \mathrm{l} 3 \mathrm{M}$ sodium acetate, $\mathrm{pH} 6.0$ and $280 \mu 1100 \%$ ethanol and subsequently stored at $-80^{\circ} \mathrm{C}$ for $5 \mathrm{~min}$ to precipitate nucleic acids. After a centrifugation step for $5 \mathrm{~min}$ at $14000 \mathrm{rpm}$ the supernatant was discarded and the pellet containing the nucleic acids was washed with $300 \mu \mathrm{l} 70 \%$ ethanol. Following an additional centrifugation step (5 min; $14000 \mathrm{rpm}$ ) the pellet was dried at room temperature and finally dissolved in 50-100 $\mu 1 \mathrm{TE}-$ buffer and $0.5-1 \mu \mathrm{l}$ Rase $(10 \mu \mathrm{g} / \mu \mathrm{l}) .1 \mu \mathrm{l}$ of this solution was used as a template for further PCR analysis.

$\underline{\text { Breaking buffer }}$

$\begin{array}{ll}\text { Tris-HCl, pH } 8.0 & 10 \mathrm{mM} \\ \mathrm{NaCl} & 100 \mathrm{mM} \\ \text { EDTA } & 1 \mathrm{mM} \\ \text { SDS } & 1 \%(\mathrm{w} / \mathrm{v}) \\ \text { Triton X-100 } & 2 \%(\mathrm{v} / \mathrm{v})\end{array}$

\subsubsection{DNA sequencing}

DNA sequence analysis was performed according to Sanger et al. ${ }^{[99]}$ by GATC Biotech AG, Konstanz. The results obtained by sequencing were compared to the corresponding entries in the Uniprot database. 


\subsection{Protein chemistry and analytics}

\subsubsection{Purification of the yeast 20S proteasome}

Purification of wt and mutant yCPs was carried out together with the technician Richard Feicht according to published procedures ${ }^{[100]}$. $120 \mathrm{~g}$ yeast cells were solubilized in approximately $150 \mathrm{ml}$ of $50 \mathrm{mM} \mathrm{KH} \mathrm{PO}_{4} / \mathrm{K}_{2} \mathrm{HPO}_{4}$ buffer $\mathrm{pH} 7.5$. DNAse I was added and the cells were disrupted with a French press. The cell lysate was centrifuged for $30 \mathrm{~min}$ at $21000 \mathrm{rpm}$ at $4{ }^{\circ} \mathrm{C}$. The resulting supernatant was filtered and $30 \%$ of saturated ammonium sulfate was added. Subsequently, the cell lysate was loaded on a Phenyl Sepharose ${ }^{\mathrm{TM}} 6$ Fast Flow column pre-equilibrated with $1 \mathrm{M}$ ammonium sulfate in $20 \mathrm{mM} \mathrm{KH_{2 }} \mathrm{PO}_{4} / \mathrm{K}_{2} \mathrm{HPO}_{4}$ buffer $\mathrm{pH}$ 7.5. The yCP was eluted by applying a linear gradient from $1 \mathrm{M}$ to $0 \mathrm{M}$ ammonium sulfate in 4 column volumes. Collected fractions were tested for proteolytic activity using the fluorogenic substrates Suc-Leu-Leu-Val-Tyr-AMC (for wt yCP) and Cbz-Leu-Leu-Glu-AMC (for $\beta 5$ mutant yCPs): $30 \mu \mathrm{l}$ of each fraction were incubated for $1 \mathrm{~h}$ with $1 \mu \mathrm{l}$ of $10 \mathrm{mM}$ substrate and the resulting fluorescence was measured $\left(\lambda_{\text {exc }}=360 \mathrm{~nm}\right.$ and $\left.\lambda_{\text {em }}=460 \mathrm{~nm}\right)$. Active fractions were pooled and applied to a hydroxyapatite column, which has been equilibrated with $20 \mathrm{mM} \mathrm{KH} \mathrm{KH}_{4} / \mathrm{K}_{2} \mathrm{HPO}_{4} \mathrm{pH}$ 7.5. Using a linear gradient from $20 \mathrm{mM}$ to $500 \mathrm{mM}$ $\mathrm{KH}_{2} \mathrm{PO}_{4} / \mathrm{K}_{2} \mathrm{HPO}_{4}$ in 20 column volumes, the yCP was eluted. Proteolytically active fractions were loaded on a Resource Q column and a sodium chloride gradient from $0 \mathrm{mM}$ to $500 \mathrm{mM}$ in $20 \mathrm{mM}$ Tris- $\mathrm{HCl} \mathrm{pH} 7.5$ was run over 10 column volumes. For crystallization the buffer was exchanged for $10 \mathrm{mM}$ 2-(N-morpholino)ethanesulfonic acid (Mes) pH 6.8 using a HiPrep $^{\mathrm{TM}} 26 / 10$ desalting column.

\subsubsection{Purification of the murine immuno- and constitutive $20 S$ proteasome}

Immuno- and constitutive proteasome samples were kindly provided by Dr. Michael Basler and Ricarda Schwab from the group of Prof. Dr. Marcus Groettrup, University of Constance, Department of Biology/Immunology, Universitätsstr. 10, Constance, DE. The cCP was purified from livers of C57BL/6 PSMB8 ( $\beta 5 \mathrm{i})$ and PSMB10 ( $\beta 2 \mathrm{i})$ knockout mice according to published protocols ${ }^{[101]}$. iCP samples were prepared from livers of BALB/c mice eight days after their intravenous infection with 200 plaque-forming units of lymphocytic choriomeningitis virus strain WE (LCMV-WE) ${ }^{[101]}$. LCMV-WE selectively infects liver cells and leads to an almost complete conversion of the $\mathrm{cCP}$ to the $\mathrm{iCP}$ within eight days, when mice were sacrificed and livers removed. Purification of the iCP was carried out according to 
established procedures $^{[101]}$. For short-term storage and subsequent crystallisation trials samples were kept at $4{ }^{\circ} \mathrm{C}$ (in $10 \mathrm{mM}$ Hepes $\mathrm{pH} 7.2,300 \mathrm{mM} \mathrm{KCl}, 5 \mathrm{mM} \mathrm{MgCl} 2$ ), otherwise the protein was frozen at $-80^{\circ} \mathrm{C}$.

\subsubsection{SDS polyacrylamide gel electrophoresis}

Discontinuous SDS-PAGE was performed according to Laemmli ${ }^{[102]}$. Gels were run in $1 \mathrm{x}$ running buffer at $25 \mathrm{~mA}$ per gel until the front line has passed the bottom of the gel. SDS polyacrylamide gels were stained for $30 \mathrm{~min}$ with Coomassie solution and afterwards destained.

\begin{tabular}{lll}
\hline Compound & Separating gel (12 \%) & Stacking gel (4\%) \\
\hline Acrylamide & $6 \mathrm{ml}$ & $1 \mathrm{ml}$ \\
Separating gel buffer & $5 \mathrm{ml}$ & - \\
Stacking gel buffer & - & $5 \mathrm{ml}$ \\
$\mathrm{H}_{2} \mathrm{O}$ & $9 \mathrm{ml}$ & $4 \mathrm{ml}$ \\
APS $(10 \%(\mathrm{v} / \mathrm{v}))$ & $100 \mu \mathrm{l}$ & $100 \mu \mathrm{l}$ \\
TEMED $(1 \%(\mathrm{v} / \mathrm{v}))$ & $10 \mu \mathrm{l}$ & $10 \mu \mathrm{l}$ \\
\hline
\end{tabular}

Table 11 Pipetting scheme for four SDS gels.

$\underline{\text { Acrylamide solution }}$

SDS-PAGE loading dye

Separating gel buffer

$\underline{\text { Stacking gel buffer }}$
Acrylamide
Bisacrylamide

$39 \%(\mathrm{w} / \mathrm{v})$

Tris-HCl, pH 6.8

Glycerol

Saccharose

SDS

Bromphenol blue

$\beta$ Mercaptoethanol

Tris-HCl, pH 8.8

SDS

Tris-HCl, pH 6.8

SDS
$1.2 \%(\mathrm{w} / \mathrm{v})$

$60 \mathrm{mM}$

$30 \%(\mathrm{v} / \mathrm{v})$

$10 \%(\mathrm{w} / \mathrm{v})$

$5 \%(\mathrm{w} / \mathrm{v})$

$0.02 \%(\mathrm{w} / \mathrm{v})$

$3 \%(\mathrm{v} / \mathrm{v})$

$1.5 \mathrm{M}$

$0.4 \%(\mathrm{w} / \mathrm{v})$
$0.5 \mathrm{M}$

$0.4 \%(\mathrm{w} / \mathrm{v})$ 
$\underline{\text { Running buffer }}$

Tris-HCl, $\mathrm{pH}$ 8.2-8.3

$25 \mathrm{mM}$

Glycine

$192 \mathrm{mM}$

SDS

$0.1 \%(\mathrm{w} / \mathrm{v})$

$\underline{\text { Staining solution }}$

Coomassie Brilliant Blue R250

$0.05 \%(\mathrm{w} / \mathrm{v})$

Isopropanol

$25 \%(\mathrm{v} / \mathrm{v})$

Glacial acetic acid

$10 \%(\mathrm{v} / \mathrm{v})$

$\underline{\text { Destaining solution }}$

Glacial acetic acid

$10 \%(\mathrm{v} / \mathrm{v})$

\subsubsection{Determination of protein concentration}

UV/VIS spectra of protein solutions were recorded with the nanophotometer of IMPLEN. The absorption at a wavelength of $280 \mathrm{~nm}$ was used to calculate protein concentrations via the Lambert-Beer law. The required theoretical molar extinction coefficient was computed with the ProtParam tool (yCP: $727.3 \mathrm{~cm} \cdot 1 / \mathrm{mmol}$, cCP: $685.0 \mathrm{~cm} \cdot 1 / \mathrm{mmol}$, iCP: $678.8 \mathrm{~cm} \cdot 1 / \mathrm{mmol}$ ).

\subsection{Proteasome activity tests}

\subsubsection{Overlay assay}

Yeast strains were streaked as patches on YPD plates and incubated for two days at $30{ }^{\circ} \mathrm{C}$. Next, cells were replica plated on a steril filter paper lying on an YPD plate and grown for $48 \mathrm{~h}$ at $30^{\circ} \mathrm{C}$. The filter was placed in a glass Petri dish and the cells were lysed with $10 \mathrm{ml}$ of chloroform for $15 \mathrm{~min}$. After having dried the filter in a plastic petri dish $10 \mathrm{ml}$ of hand warm overlay solution (1\% (w/v) agar, 50 mM Tris-HCl pH 8.0, $300 \mu \mathrm{l}$ of 10 mM Cbz-Gly-GlyLeu-pNA substrate in DMSO) were added. Incubation for $3 \mathrm{~h}$ at $30{ }^{\circ} \mathrm{C}$ was followed by exposure to $10 \mathrm{ml}$ of $0.1 \%(\mathrm{w} / \mathrm{v})$ sodium nitrite solution in $1 \mathrm{M} \mathrm{HCl}$ for $5 \mathrm{~min}$. Next, this solution was exchanged for $10 \mathrm{ml}$ of $0.5 \%(\mathrm{w} / \mathrm{v})$ ammonium sulfamate solution in $1 \mathrm{M} \mathrm{HCl}$ and after 5 min again discarded. Finally, the filter was stored in $10 \mathrm{ml}$ of $0.05 \%(\mathrm{w} / \mathrm{v}) \mathrm{N}-(1-$ naphthyl)ethylenediamine solution in $47 \%$ (v/v) EtOH for $10-30 \mathrm{~min}$. Cells with normal ChTL activity became pink, while those with defects were less coloured. 


\subsubsection{Fluorescence activity test and determination of $\mathrm{IC}_{50}$ values}

Proteasome activity and the inhibitory potency of ligands are routinely determined by fluorescence spectroscopy using AMC-peptides as substrates. CPs were mixed with a series of different inhibitor concentrations or DMSO as a control in $100 \mathrm{mM}$ Tris- $\mathrm{HCl}$, pH 7.4 and samples were stored for $45 \mathrm{~min}$ at room temperature to allow inhibitor binding. Next, the $\beta 5$ specific substrate Suc-Leu-Leu-Val-Tyr-AMC was added to a final concentration of $200 \mu \mathrm{M}$. During the subsequent $60 \mathrm{~min}$ incubation at room temperature residual proteasome activity hydrolysed the substrate and released the AMC-fluorophor. After dilution (1:10) of the samples with $20 \mathrm{mM}$ Tris-HCl, $\mathrm{pH} 7.4$, which strongly slowed down the reaction, the relative fluorescence units (RFU) were measured with a Varian Cary Eclipse Fluorescence Spectrophotometer (Agilent Technologies) at $\lambda_{\mathrm{exc}}=360 \mathrm{~nm}$ and $\lambda_{\mathrm{em}}=460 \mathrm{~nm}$. RFU values were normalized to the DMSO treated control, which should have retained nearly $100 \%$ activity. The calculated residual activities were plotted against the logarithm of the applied inhibitor concentration and fitted to the equation $\mathrm{Y}=$ Bottom $+($ Top-Bottom $) /\left(1+10^{\wedge}((\log \mathrm{IC} 50-\right.$ $\mathrm{X})^{*}$ HillSlope)) (X: logarithm of inhibitor concentration; Y: Residual enzymatic activity; $\log$ (inhibitor) vs response - variable slope (four parameters), GraphPad Prism 5). The $\mathrm{IC}_{50}$ value, the ligand concentration that leads to $50 \%$ inhibition of the enzymatic activity, was deduced from the fitted data.

\subsection{Protein crystallography}

\subsubsection{Crystallization of the yeast 20S proteasome}

Purified yCP was concentrated to approximately $40 \mathrm{mg} / \mathrm{ml}$ at $5000 \mathrm{rpm}$ using an Amicon ${ }^{\circledR}$ Ultra-15 Centrifugal Filter device of $100 \mathrm{kDa}$ cutoff. Crystals of the yCP were grown at $20{ }^{\circ} \mathrm{C}$ by the hanging drop vapour diffusion method in drops containing a 1:1 mixture of reservoir and protein solution. The reservoir solution $(300 \mu \mathrm{l})$ was composed of $25 \mathrm{mM}$ magnesium acetate, $100 \mathrm{mM}$ Mes pH 6.8 and 9-13\% (v/v) 2-methyl-2,4-pentanediol (MPD). Crystals were cryoprotected by the addition of $5 \mu \mathrm{l}$ of $20 \mathrm{mM}$ magnesium acetate, $100 \mathrm{mM}$ Mes, $\mathrm{pH}$ 6.8 and $30 \%(\mathrm{v} / \mathrm{v})$ MPD and immediately frozen in liquid nitrogen. Crystal soaking experiments were performed by supplementing crystal drops with $5 \mu 1$ cryoprotection solution and $0.3-3 \mathrm{mM}$ of inhibitor for 2 to 24 hours. Afterwards crystals were super-cooled in liquid nitrogen. 


\subsubsection{Crystallization of the murine 20S proteasomes}

Protein samples were concentrated to approximately $30 \mathrm{mg} / \mathrm{ml}$ at $5000 \mathrm{rpm}$ using Millipore centrifugal devices with a Cut-off of $30 \mathrm{kDa}$. Initial crystallization trials with the murine cCP and iCP were set up in 96-well sitting drop plates with a final drop size of $0.2 \mu 1$ containing a protein:reservoir ratio of 1:1. Hanging drop plates were set up with final drop volumes of $1 \mu 1$. All plates were stored at $20{ }^{\circ} \mathrm{C}$ and crystals grew from MPD conditions within a few days. The iCP crystallized from a reservoir solution containing $0.2 \mathrm{M}$ sodium iodide and $40 \%$ MPD, whereas cCP crystals preferentially grew from $0.2 \mathrm{M}$ sodium formate or $0.2 \mathrm{M}$ potassium acetate and $40 \%$ MPD. After addition of 1 to $5 \mu$ of reservoir solution $\mathrm{cCP}$ and iCP crystals were frozen in liquid nitrogen. cCP and iCP complex structures with ONX 0914 were obtained by soaking crystals with $3 \mathrm{mM} \mathrm{ONX} 0914$ (dissolved in DMSO) in 1-5 $\mu \mathrm{l}$ of reservoir solution for at least $8 \mathrm{~h}$ prior to freezing.

\subsubsection{Data collection, processing and structure determination}

Diffraction data were collected at the Swiss Light Source, Villigen, Switzerland at the beamlines X06SA (Pilatus 6M detector) and X06DA (MAR CCD detector) at a wavelength of $1.0 \AA$ A. For all data sets cryoprotection $(100 \mathrm{~K})$ was used. Indexing, integration and scaling of the obtained data was performed with the program package $\mathrm{XDS}^{[103]}$. All proteasome structures were solved by Patterson search calculations with PHASER using the maximum likelihood method ${ }^{[104]}$. For yCP:ONX 0914 complex structures the yCP apo crystal structure (PDB ID 1RYP ${ }^{[13]}$ ) served as a starting model and structure determination was performed as previously published ${ }^{[105]}$. Structure elucidation of the $\mathrm{CCP}$ and $\mathrm{iCP}$ was carried out by molecular replacement with the coordinates of the bovine cCP (PDB ID 1IRU ${ }^{[21 \mathrm{a}]}$ ). For rigid body, TLS (Translation/Libration/Screw) and positional refinements REFMAC5 ${ }^{[106]}$ was used. 4-Fold and 2-fold non-crystallographic symmetry averaging was applied for the cCP and iCP in refinement and model building, respectively. Model building was performed with the interactive three-dimensional graphic programs $\mathrm{MAIN}^{[88]}$ and $\mathrm{COOT}^{[87]}$. Waters were placed with Arp/Warp ${ }^{[86]}$. Ligands were built with Sybyl ${ }^{[91]}$ and their topology and parameter files were created by Sketcher ${ }^{[86]}$. Graphical illustration of crystal structures was performed with the programs MOLSCRIPT ${ }^{[89]}$, BOBSCRIPT ${ }^{[85]}$ and PyMOL ${ }^{[90]}$. Conolly surfaces were calculated and depicted with GRASP ${ }^{[107]}$. The final coordinates were proven to have good stereochemistry according to the Ramachandran plot by using Procheck ${ }^{[86]}$ as well as 
reasonable $R_{\text {work}}$, $R_{\text {free }}$, r.m.s.d. bond and angle values (see Table 12 and Table 13). The coordinates of published structures were deposited in the PDB under the following accession codes: 3UNH (iCP), 3UNE (cCP), 3UNF (iCP:ONX 0914), 3UNB (cCP:ONX 0914), 3UN8 (yCP:ONX 0914_ep), 3UN4 (yCP:ONX 0914_mo). 


\section{Results}

\subsection{Sequence alignments}

The quaternary and tertiary structure of the 20S proteasome is strictly conserved from archaea to mammals and it is assumed that its two types of subunits, termed $\alpha$ and $\beta$, evolved from a common ancestor protein ${ }^{[12-14,21 a, 32]}$. In this regard the catalytically active proteasome subunits of murine $20 \mathrm{~S}$ proteasome types display high sequence identities to each other: $\beta 1 \mathrm{c} / \beta 1 \mathrm{i}: 63.3 \%, \beta 2 \mathrm{c} / \beta 2 \mathrm{i}: 58.9 \%, \beta 5 \mathrm{c} / \beta 5 \mathrm{i}: 72.4 .0 \%, \beta 5 \mathrm{c} / \beta 5 \mathrm{t}: 54.4 \% \beta 5 \mathrm{i} / \beta 5 \mathrm{t}: 50.5 \%$ (Figure 8). Although sequence alignments are indicative of an overall conserved fold for all proteolytically active subunits, defined differences in their substrate binding channels give rise to different cleavage preferences in the $\mathrm{cCP}$, iCP and $\mathrm{tCP}^{[13,21 \mathrm{c}]}$. In particular, hydrophobic amino acids in the unprimed substrate binding pockets of subunit $\beta 1 \mathrm{i}$ have been predicted to attenuate the CL activity and to increase the ChTL activity ${ }^{[13]}$. In contrast, the sequence analysis of the subunits $\beta 2 \mathrm{c} / \mathrm{i}$ and $\beta 5 \mathrm{c} / \mathrm{i}$ provided no hints on changes in the chemical properties of their substrate binding channels and on profound differences in their substrate specificities (Figure 8). However, for the vertebrate-specific subunit $\beta 5 \mathrm{t}$, which is exclusively expressed in cTECs $^{[35 b]}$, the primary sequence of its substrate binding channel is strongly altered. In particular, the polarity of the binding pockets appears to be dramatically increased, thereby leading to a markedly reduced ChTL activity and to a different peptide product pattern of the tCP (Figure 8C; see also chapter 6.4.3). 
A

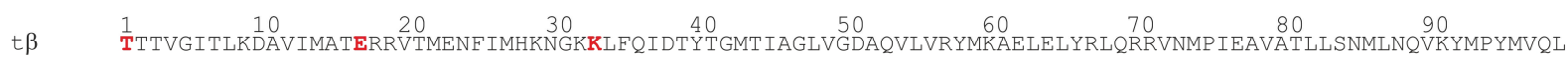
Y 11 TSIMAVTFKDGVILGADSRTTTGAYIANRVTDKLTRVHDK IWCCRSGSAADTQAIADIVQYHLELYYSSQYGTP. STETAASVEKELCYENKD. NLTAGI

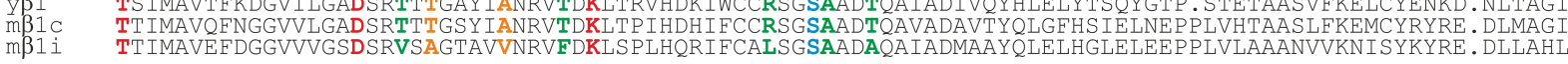

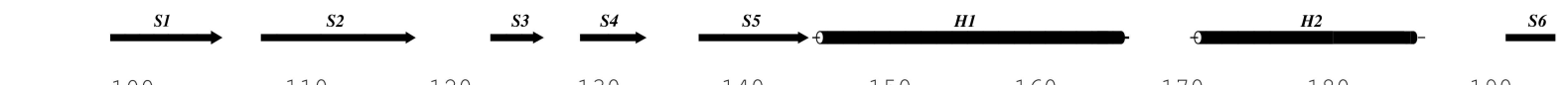
t 1 LVGGIDTA..PHVFSIDAAGGSVEDIYASTGSGSPFVYGVLESQYSEKMTVDEGVDLVIRAISAAKQRDSASGGMIDVAVITRKDGYVQ.LPTDQIESR

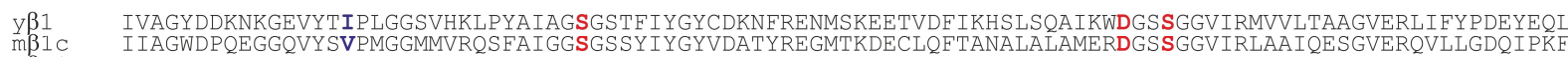
mß1i IVAGWDQREGGQVYG. TMGGMLIRQPFTIGGSGSSY IYGYVDAAYKPGMTPEECRRFTTNAITLAMNRDGSSGGVIYLVTITAAGVDHRVILGDELPKE

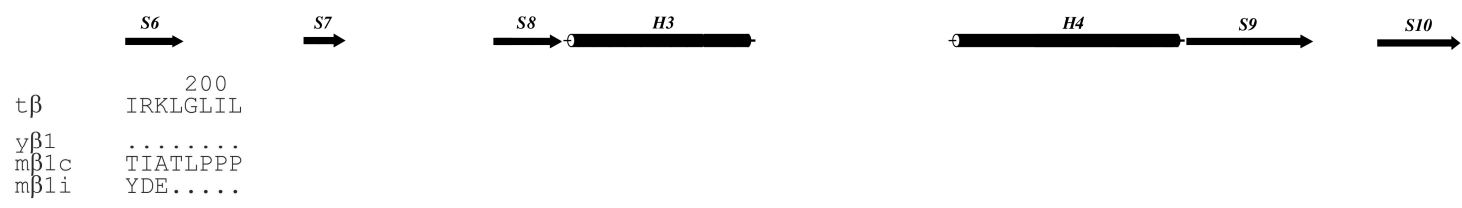

B

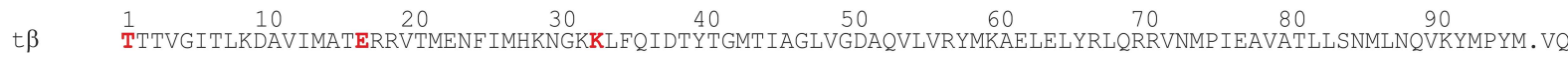

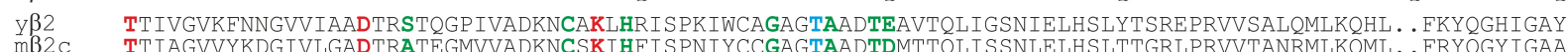

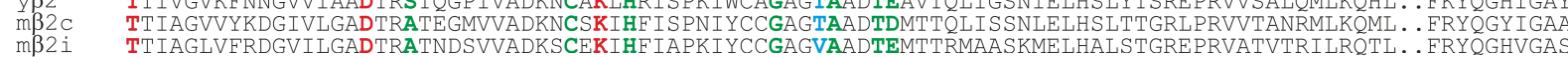

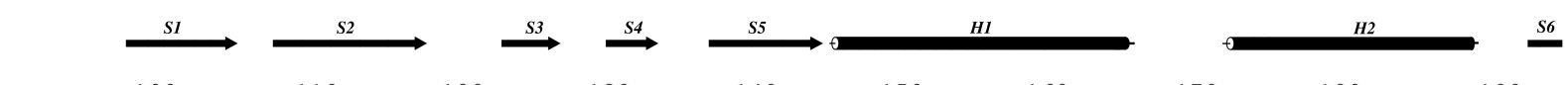

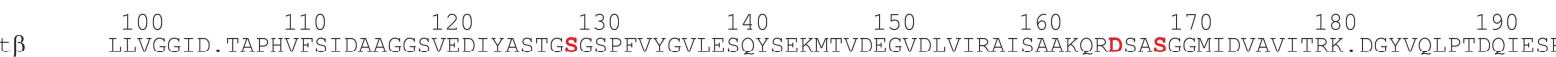
y 32 LIVAGVDPTGSHLFSIHAHGSTDVGYYLSLGSGSLAAMAVLESHWKODLTKEEAIKLASDAIOAGIWNDLGSGSNVDVCVMEIGKDAEYLRNYLTPNVR m $32 \mathrm{C}$ LVLGGVDVTGPHLYSIYPHGSTDKLPYVTMGSGSLAAMAVFEDKFRPDMEEEEAKKLVSEAIAAGIFNDLGSGSNIDLCVISKS.KLDFLRPFSVPNKK m $32 i$ LVVGGVDLNGPQLYEVHPHGSYSRLPFTALGSGQGAAVALLEDRFQPNMTLEAAQELLVEAITAGILSDLGSGGNVDACVITAG. GAKLQRALSTPTEP

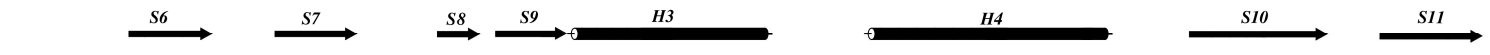

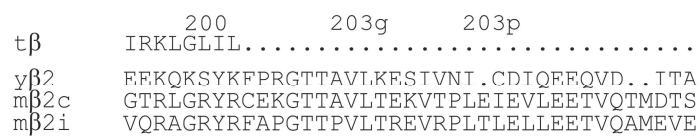

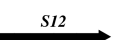

C

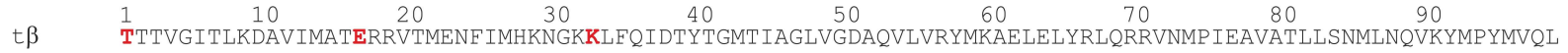
y 35 TTTLAFRFQGGIIVAVDSRATAGNWVASQTVKKVIEINPFLLGTMAGGAADCQFWETWLGSQCRLHELREKERISVAAASKILSNLVYQYKGAGLSMGT $\mathrm{m} \beta 5 \mathrm{C}$ TTTLAFKFLHGVIVAADSRATAGAYIASQTVKKVIEINPYLLGTMAGGAADCS FWERLLARQCRIYE LRNKERISVAAASKLLANMVYQYKGMGLSMGT $\begin{array}{ll}\text { m } 35 i & \text { TTTLAFKFOHGVIVAVDSRATAGSY ISSLRMNKVIEINPYLIGTMSGCAADCOYWERLLAKECRLYY IRNGERISVSAASKLLSNMMLOYRGMGLSMGS } \\ \text { m35t } & \text { TTTLAFRFRHGVIAAADTRSSCGSYVACPASRKVIPVHQRLIGTTSGTSADCATWYRVLRRELRLRELREGQLPSVAGTAKLLAAMMSCYRGLDLCVAT }\end{array}$

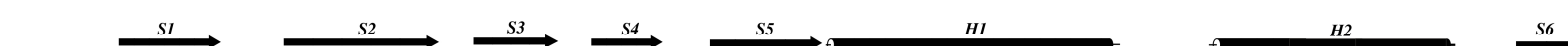

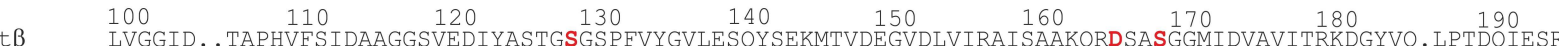
yß5 MICGYTRKEGPTIYYVDSDGTRLKGDIFCVGSGQTFAYGVLDSNYKWDLSVEDALYLGKRSILAAAHRDAYSGGSVNLYHV. TEDGWIYHGNHDVGELE $\mathrm{m} \beta 5 \mathrm{C}$ MICGWD. KRGPGLYYVDSEGNRISGTAFSVGSGSVYAYGVMDRGYSYDLKVEEAYDLARRAIYQATYRDAYSGGAVNLYHV. REDGWIRVSSDNVADL

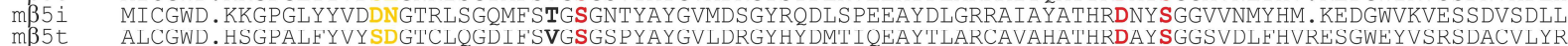
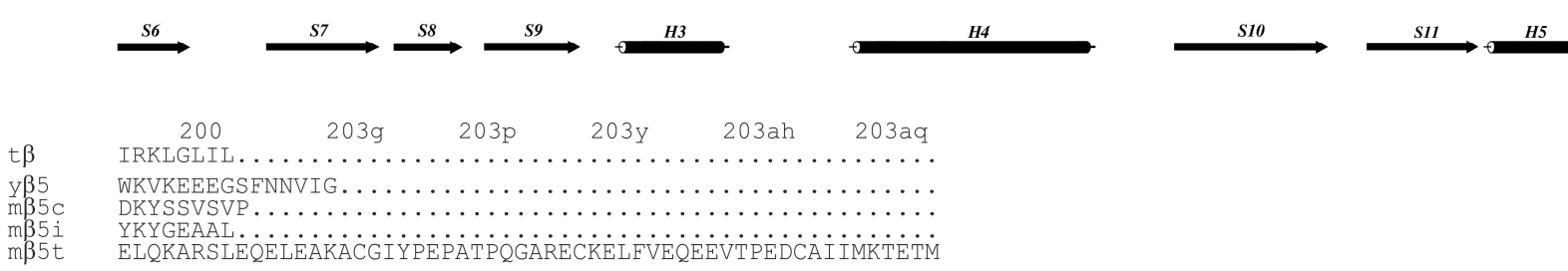

Figure 8 Sequence alignments of active $\beta$ subunits from archaeal $(t)$, yeast $(y)$ and murine $(m) 20 S$ proteasomes. Protein sequences for $(\mathbf{A}) \beta 1,(\mathbf{B}) \beta 2$ and $(\mathbf{C}) \beta 5$ subunits are given in the one-letter code. Amino acids are numbered according to the $\beta$ subunit of the $20 \mathrm{~S}$ protesomes from the archaeon T. acidophilum (t); insertions are indicated by lower-case letters. Helices and sheets are depicted for the i subunits. Residues that are crucial for the catalytic activity are marked in red. The substrate specificity pocket forming amino acids are coloured in green $(\mathrm{S} 1)$, blue $(\mathrm{S} 2)$, orange $(\mathrm{S} 3)$ and yellow $\left(\mathrm{S}^{\prime}\right)$, respectively. Residue 113 is absent in all $\beta 1 \mathrm{i}$ subunits (purple). Amino acids that are suggested to influence the active site architecture and proteolytic activity of subunit $\beta 5$ are highlighted in black.

Adapted from Huber et al., 2012 ${ }^{[32]}$. 


\subsection{X-ray structures of the mouse $20 \mathrm{~S}$ immuno- and constitutive proteasome}

\subsubsection{Crystallization and structure determination}

Infection of BALB/c mice with LCMV-WE leads to an efficient (95\%) conversion of cCPs to iCPs in the murine livers within eight days. The synthesized iCPs were purified according to published procedures ${ }^{[101]}$. The same purification procedure was applied to livers of uninfected gene targeted $\beta 2 \mathrm{i}^{-/-}$and $\beta 5 \mathrm{i}^{-/-} \mathrm{C} 57 \mathrm{BL} / 6$ mice and yielded pure cCP samples (Figure 9).
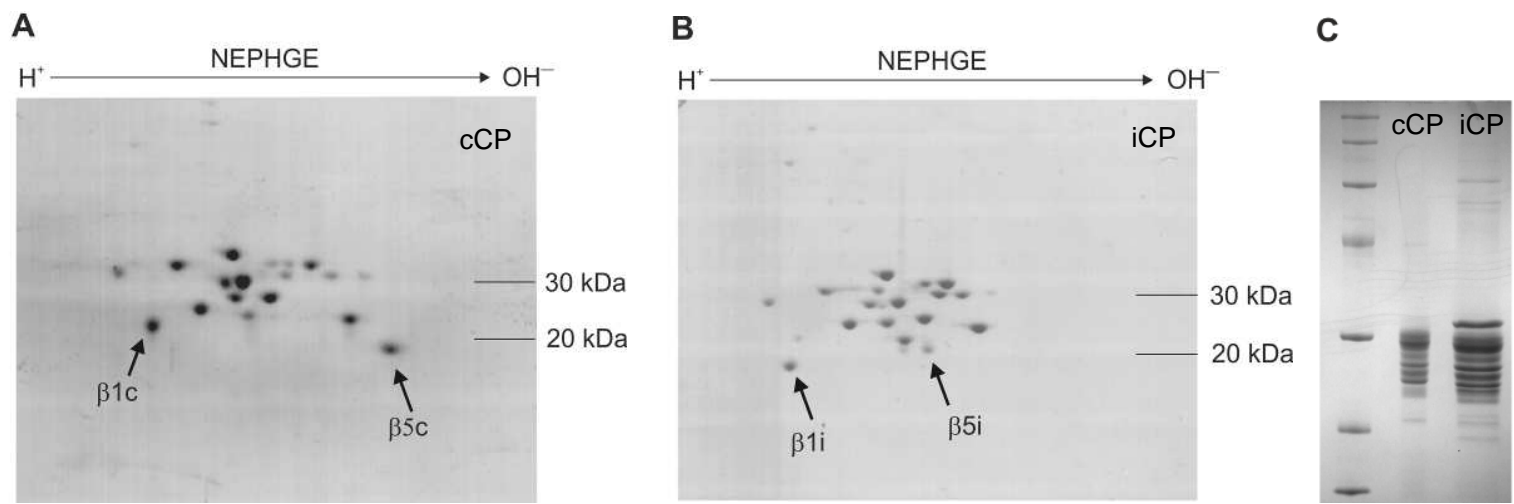

Figure 9 Coomassie-stained gels of purified murine cCP and iCP samples used for crystallization trials. 2D non-equilibrium $\mathrm{pH}$ gradient gel electrophoresis (NEPHGE)/SDS-PAGE gels of (A) $80 \mu \mathrm{g}$ of purified cCP isolated from livers of $\beta 2 \mathrm{i}^{-/-} \beta 5 \mathrm{i}^{--}$gene targeted mice and (B) $80 \mu \mathrm{g}$ of iCP from livers of LCMV-WE infected $\mathrm{BALB} / \mathrm{c}$ mice. Proteasome subunits were assigned according to Groettrup et al., $1996^{[21 \mathrm{~b}]}$. (C) One-dimensional SDS-PAGE analysis of cCP and iCP samples.

Adapted from Huber et al., 2012 $2^{[32]}$.

In a joint collaboration with the group of Prof. Dr. Marcus Groettrup of the University of Constance, Dr. Michael Basler and Ricarda Schwab prepared appropriate amounts of murine iCP and cCP for crystallographic analysis. Samples were proven for their purity by 2D-PAGE (Figure 9), concentrated to approximately $30 \mathrm{mg} / \mathrm{ml}$ and subsequently subjected to initial sitting drop vapour diffusion crystallization trials. A sparse matrix screen of about 400 conditions was tested with a protein:reservoir ratio of 1:1 and a final drop volume of $0.2 \mu 1$. Both CPs crystallized within a few days from various reservoir solutions containing $40 \%$ MPD and different salts (Figure 10A). The obtained crystals were exposed to synchrotron Xray radiation and their diffraction patterns were analysed (Figure 10B). Albeit their small size, crystals diffracted to a resolution of approximately $6 \AA$ A. However, due to disordered crystal lattices most diffractions patterns were not suitable for structure analysis. Crystal quality was improved by avoiding freezing of the protein samples, by filtering samples upon concentration and by extensive screening of crystallization conditions. Finally, high-quality crystals of the $\mathrm{cCP}$ and $\mathrm{iCP}$ with resolution limits of $3.2 \AA$ were obtained (Table 12). After the successful collection of diffraction data for the cCP and iCP apo-structures, inhibitor soaking 
experiments with the iCP-selective compound ONX 0914 were undertaken. Crystals of the cCP and iCP were exposed to ONX 0914 in a final concentration of $3 \mathrm{mM}$ for at least $8 \mathrm{~h}$. Diffraction data of both cCP:ONX 0914 and iCP:ONX 0914 crystals were collected to a resolution limit of $2.9 \AA$ (Table 12) and data processing was performed according to section 5.5.3.

A
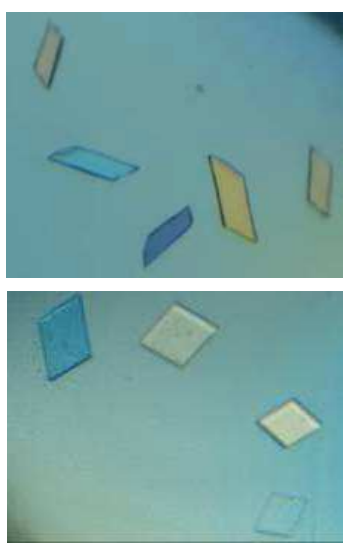

B

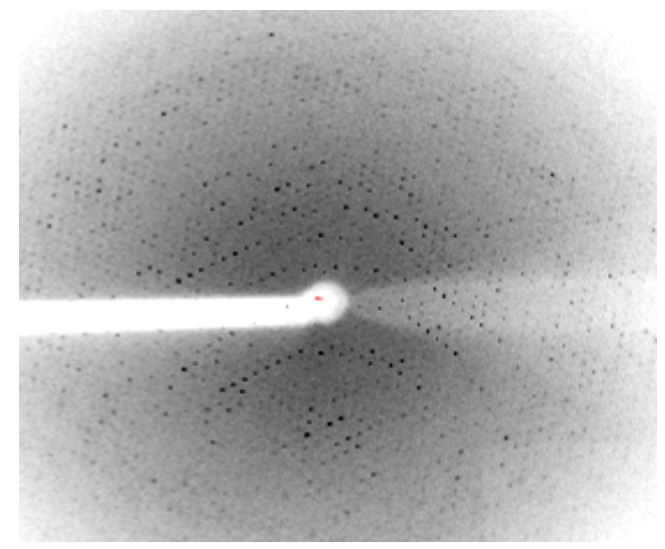

Figure 10 Analysis of murine $\mathrm{CCP}$ and iCP crystals for X-ray diffraction. (A) Birefringent murine $\mathrm{cCP}$ and iCP crystals; (B) Diffraction pattern of an iCP crystal.

The X-ray data revealed for both cCP and iCP crystals space group $\mathrm{P} 2{ }_{1}$, but different unit cell parameters. Calculation of the Matthews coefficient indicated a solvent content of about $50 \%$, assuming that the asymmetric unit of iCP crystals contained only one $\mathrm{CP}$, whereas the asymmetric unit of cCP crystals was built up of two 20S proteasomes. The same applied to both ligand complex structures (Table 12). All four crystal structures could be finalized with $\mathrm{R}_{\text {free }}$ values below $27.5 \%$ and root-mean-square deviations for bond lengths and bond angles of less than $0.005 \AA$ and $0.93^{\circ}$.

A

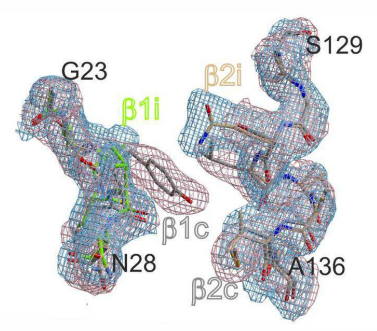

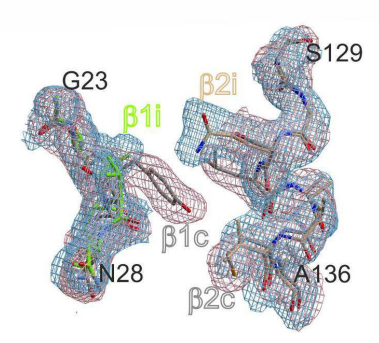

B

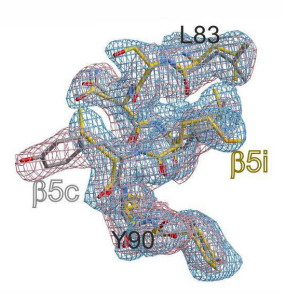

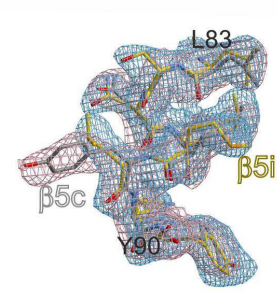

Figure 11 Stereo representation of the electron density maps of the murine $\mathrm{CCP}$ and $\mathrm{ICP}$. For the calculation of the $2 \mathrm{~F}_{\mathrm{O}}-\mathrm{F}_{\mathrm{C}}$ electron density maps (iCP: blue mesh; $\mathrm{cCP}$ : red mesh), which are contoured to $0.8 \sigma$, the phases of the displayed amino acids have been omitted. The electron density sections clearly depict explicit differences in the amino acid sequences of $\mathrm{c}$ and i subunits: (A) Tyr25 of subunit $\beta 1 \mathrm{c}$ corresponds to Ala25 in subunit $\beta 1$ i; and Ser131, Leu132 as well as Met135 of subunit $\beta 2 \mathrm{c}$ are substituted by Gln, Gly and Val in subunit $\beta 2 \mathrm{i}$, respectively. (B) Tyr88 of subunit $\beta 5 \mathrm{c}$ is exchanged for Leu in subunit $\beta 5 \mathrm{i}$. Adapted from Huber et al., 2012 $2^{[32]}$. 
All proteasome subunits were well defined in the $2 \mathrm{~F}_{\mathrm{O}}-\mathrm{F}_{\mathrm{C}}$ electron density maps and differences in the primary sequences of catalytically active $\mathrm{c}$ and $\mathrm{i}$ subunits were unambiguously depicted by positive and negative $\mathrm{F}_{\mathrm{o}}-\mathrm{F}_{\mathrm{c}}$ maps (Figure 11), indicating that pure iCPs and cCPs have been crystallized. Amino acid residue numbers were allocated according to the sequence alignment to the proteasomal $\beta$ subunit from T. acidophilum (Figure 8).

m_iCP $\quad$ m_iCP:ONX 0914 m_cCP $\quad$ m_cCP:ONX0914

Crystal parameters

Space group

Cell constants

$\mathrm{CPs} / \mathrm{AU}^{\mathrm{a}}$

\section{Data collection}

Beam line

Wavelength $(\AA)$

Resolution range $(\AA)^{\mathrm{b}}$

No. observations

No. unique reflections ${ }^{c}$

Completeness $(\%)^{\mathrm{b}}$

$\mathrm{R}_{\text {merge }}(\%)^{\mathrm{b}, \mathrm{d}}$

$\mathrm{I} / \sigma(\mathrm{I})^{\mathrm{b}}$

\section{Refinement \\ (REFMAC5)}

Resolution range $(\AA)$

No. refl. working set

No. refl. test set

No. non hydrogen

No. of ligand atoms

Solvent $\left(\mathrm{H}_{2} \mathrm{O}, \mathrm{K}^{+}, \mathrm{Cl}^{-}, \mathrm{J}^{-}\right)$

$\mathrm{R}_{\text {work }} / \mathrm{R}_{\text {free }}(\%)^{\mathrm{e}}$

r.m.s.d. bond $(\AA) /\left({ }^{\circ}\right)^{\mathrm{f}}$

Average B-factor $\left(\AA^{2}\right)$

$\begin{array}{ll}\mathrm{P} 2_{1} & \mathrm{P} 2_{1} \\ \mathrm{a}=118.3 \AA & \mathrm{a}=117.3 \AA \\ \mathrm{b}=205.2 \AA & \mathrm{b}=194.6 \AA \\ \mathrm{c}=161.9 \AA & \mathrm{c}=157.7 \AA \\ \beta=105.7^{\circ} & \beta=107.1^{\circ} \\ 1 & 1\end{array}$

X06DA, SLS

X06DA, SLS

1.0

1.0

30-3.2

(3.3-3.2)

356417

121329

$99.2(99.4)$

$10.4(51.5)$

$10.4(2.3)$

30-2.9

(3.0-2.9)

392915

145087

$97.1(89.8)$

$11.6(54.7)$

$8.0(2.0)$
$\mathrm{P} 2_{1}$

$\mathrm{a}=171.7 \AA$

$\mathrm{b}=198.6 \AA$

$\mathrm{c}=226.8 \AA$

$\beta=106.6^{\circ}$

2

Ramachandran Plot $(\%)^{\mathrm{g}} \quad 97.2 / 2.6 / 0.2 \quad 96.7 / 3.0 / 0.3 \quad 95.4 / 4.1 / 0.5 \quad 95.9 / 3.6 / 0.5$

Table $12 \mathrm{X}$-ray data collection and refinement statistics of murine $\mathrm{iCP}$ and $\mathrm{CCP}$ structures.

a Asymmetric unit.

b The values in parentheses of resolution range, completeness, $R_{\text {merge }}$ and $I / \sigma$ (I) correspond to the last resolution shell.

c Friedel pairs were treated as identical reflections.

${ }^{\mathrm{d}} \mathrm{R}_{\text {merge }}(\mathrm{I})=\Sigma_{\mathrm{hkl}} \Sigma_{\mathrm{j}}\left|\left[\mathrm{I}(h k l)_{\mathrm{j}}-\mathrm{I}(h k l)\right]\right| \Sigma_{\mathrm{hkl}} \mathrm{I}_{\mathrm{hkl}}$, where $\mathrm{I}(\mathrm{hkl})_{\mathrm{j}}$ is the $\mathrm{j}^{\text {th }}$ measurement of the intensity of reflection hkl and $\langle\mathrm{I}(\mathrm{hkl})\rangle$ is the average intensity.

e $\mathrm{R}=\Sigma_{\mathrm{hkl}}|| \mathrm{F}_{\mathrm{obs}}|-| \mathrm{F}_{\text {calc }}|| \Sigma_{\mathrm{hkl}}\left|\mathrm{F}_{\mathrm{obs}}\right|$, where $\mathrm{R}_{\text {free }}$ is calculated for a randomly chosen $5 \%$ of reflections, which were not used for structure refinement, and $\mathrm{R}_{\mathrm{work}}$ is calculated for the remaining reflections.

$\mathrm{f}$ Deviations from ideal bond lengths/angles.

$\mathrm{g}$ Number of residues in favoured, allowed or outlier region. 


\subsubsection{Subunit architecture of the cCP and $\mathrm{iCP}$}

The overall topologies of the $\mathrm{cCP}$ and iCP are identical except for the exchange of the catalytically active $c$ subunits $\beta 1 \mathrm{c}, \beta 2 \mathrm{c}$ and $\beta 5 \mathrm{c}$ by their $\mathrm{i}$ homologues $\beta 1 \mathrm{i}, \beta 2 \mathrm{i}$ and $\beta 5 \mathrm{i}$. Likewise the $\mathrm{yCP}$ crystal structure, the N-termini of the inactive $\alpha$ subunits of the $\mathrm{CCP}$ and iCP close the entrance to the interior of the CPs. Superposition of the $\alpha$ rings from cCP, iCP and yCP illustrates their high structural similarity (r.m.s.d. $\mathrm{C}_{\alpha}$ atoms $\alpha$ ring $<0.59 \AA$; Figure 12) and suggests that the gate opening mechanism ${ }^{[16]}$ is identical for all kind of CPs.

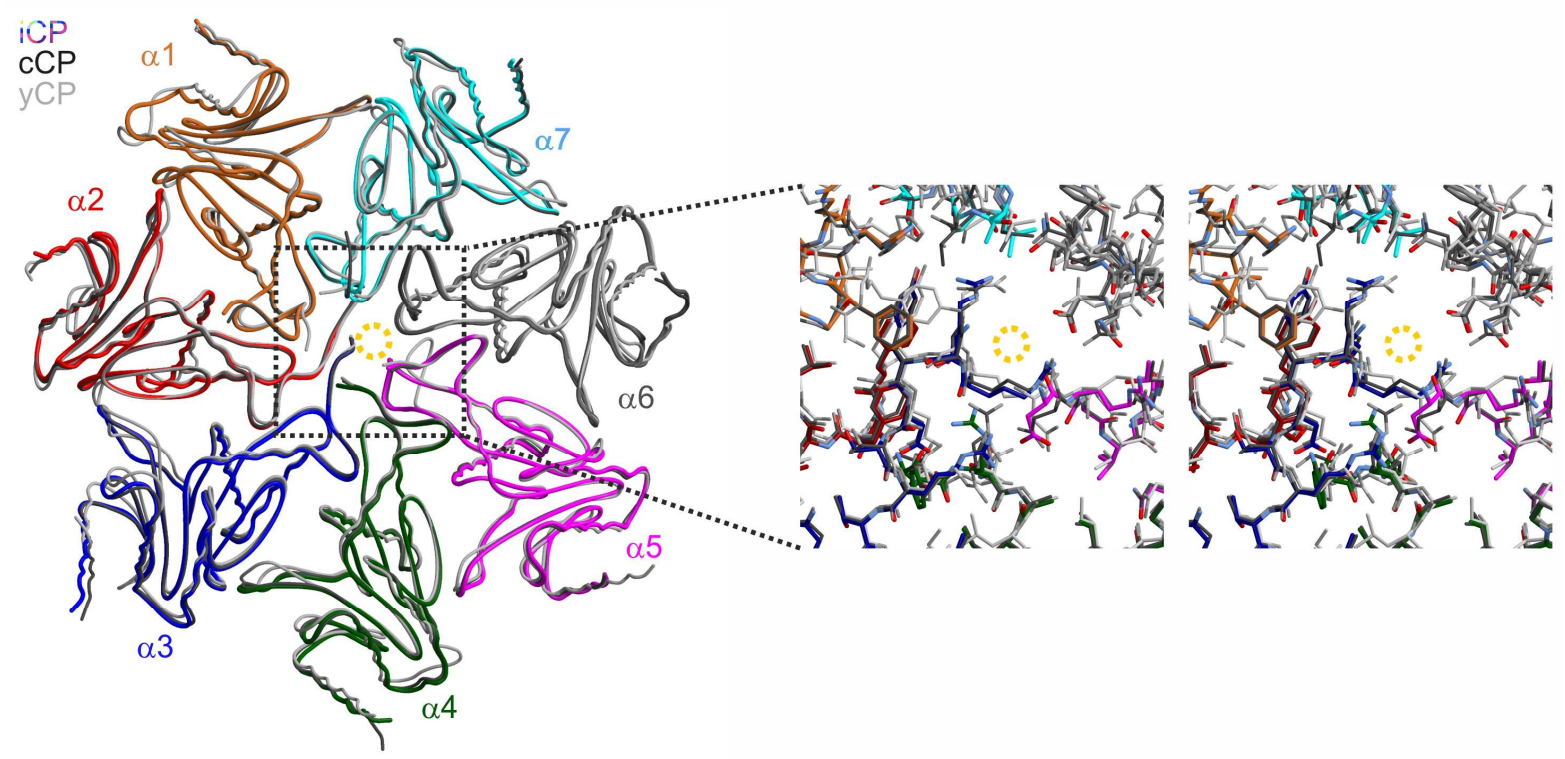

Figure 12 Structural superposition of the $\alpha$ ring of $\mathbf{y C P}, \mathbf{C C P}$ and iCP. The N-termini of the $\alpha$ subunits, in particular of $\alpha 2, \alpha 3$ and $\alpha 4$, from yCP (grey), cCP (black) and iCP (multi-coloured) prevent access into the proteolytic chamber. A stereoscopic view of the centre of the $\alpha$ ring (marked by a yellow circle) is provided. Adapted from Huber et al., 2012 $2^{[32]}$.

Subunits incorporated into both the cCP and iCP, i.e. $\alpha$ subunits and inactive $\beta$ subunits are unchanged as proven by an r.m.s.d $\mathrm{C}_{\alpha}$ atoms of $<0.35 \AA$ for their main chain tracings. Despite differences in their amino acid sequence (Figure 8) the exchangeable $\beta$ subunits adopt identical folds (r.m.s.d. $C_{\alpha}$ atoms $<0.72 \AA$ ) (Figure 13). Remarkably, also the C-terminal loop of the subunits $y \beta 2$ and $\beta 2 \mathrm{c}$, which embraces its neighbouring subunit $\beta 3$ and which is essential for CP assembly ${ }^{[108]}$, is structurally conserved in subunit $\beta 2 \mathrm{i}$.

The majority of the amino acid exchanges between the proteolytically active $\mathrm{c}$ and $\mathrm{i}$ subunits are located on the subunit surfaces and only a minority is conserved among species ( $\beta 1 \mathrm{i}: 13.6 \%, \beta 2 \mathrm{i}: 5.1 \%$, $\beta 5 \mathrm{i}: 9.8 \%$ ). As revealed by PISA analysis, the number of interaction sites between adjacent subunits of the $\mathrm{CCP}$ and the iCP differ (Table 15), but the impact on the stability or half-life of the CPs cannot be predicted from the structural data. However, the 
subunit interaction surfaces of the proteolytically active subunits and their adjacent neighbours enable the formation of mixed proteasome species, which were previously suggested to be physiologically relevant ${ }^{[29-30]}$.
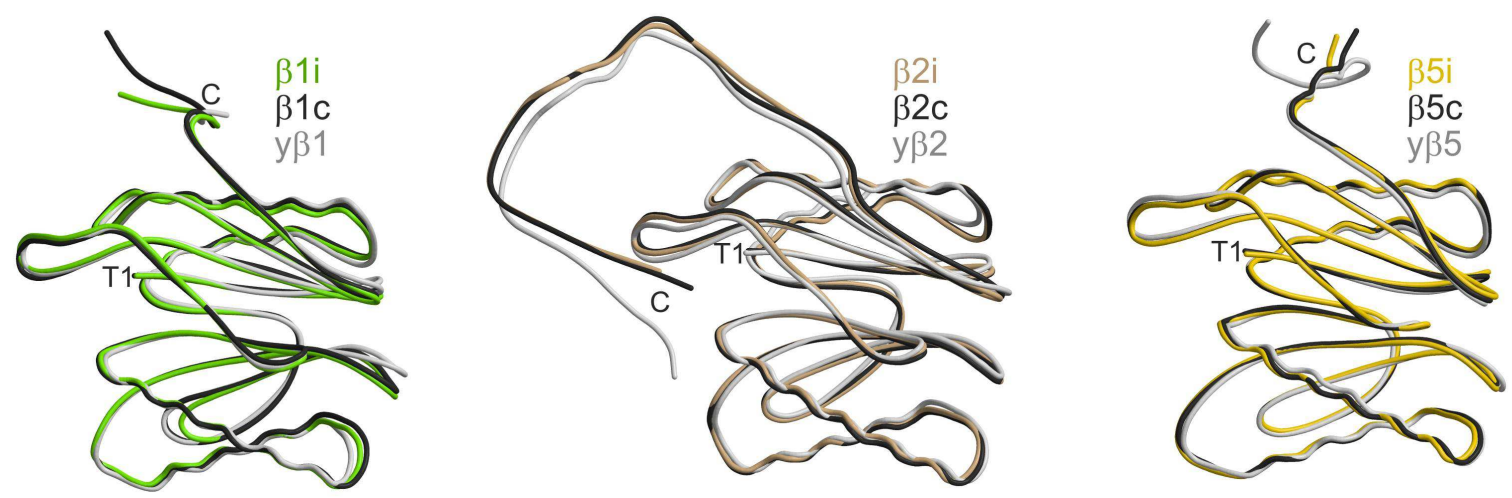

Figure 13 Main chain tracings of the proteolytically active proteasome subunits. The $\mathrm{C}_{\alpha}$ backbones of the subunits $\beta 1 \mathrm{i}$ (green), $\beta 2 \mathrm{i}$ (brown) and $\beta 5 \mathrm{i}$ (yellow) are superimposed to their constitutive counterparts from mouse (black) and yeast (grey). The nucleophilic $\mathrm{N}$-terminal Thr1 and the C-terminus are indicated.

Adapted from Huber et al., 2012 $2^{[32]}$.

\subsubsection{Structural analysis of the substrate binding channels}

The substrate and inhibitor specificities of the proteasomal active sites are predominantly determined by the enthalpic interactions of ligands with defined pockets of the primed and unprimed substrate binding channels surrounding the nucleophilic Thr1. The following section summarizes the similarities and alterations in the primed and unprimed sites of proteolytically active $\mathrm{c}$ and $\mathrm{i}$ subunits.

The primed substrate binding pockets ( $S^{\prime}$ ) of the subunits $\beta 2 \mathrm{i}$ and $\beta 2 \mathrm{c}$ differ by several amino acid exchanges. However, their impact on protein hydrolysis remains enigmatic. In the primed site of subunit $\beta 1 \mathrm{i}$ one amino acid is deleted in the loop segment 113 to 124 compared to subunit $\beta 1 \mathrm{c}$. This loop shortening is a hallmark of all $\beta 1 \mathrm{i}$ subunit sequences known to date and might affect substrate preferences. Comparison of the primed pockets in the subunits $\beta 5 \mathrm{c}$ and $\beta 5 \mathrm{i}$ depicts the amino acid substitutions S115D and E116N. Although these changes might influence substrate affinities, they are not conserved between species. Using X-ray crystallography most inhibitory compounds have been shown to target the unprimed substrate binding channels ${ }^{[22,109]}$ and thus, the $S 1, S 2$ and $S 3$ pockets are far better examined than the primed ones. The unprimed sites of the subunits $\beta 2 \mathrm{c}$ and $\beta 2 \mathrm{i}$ display high similarity to each other, except for the conserved substitutions T48V and D53E. While T48V might alter the specificity for $\mathrm{P} 2$ residues, D53E is assumed to change neither the chemical environment nor 
substrate preference (Figure 14). Interestingly, Glu53 and Thr48 are also characteristic of the yeast subunit y $\beta 2$ (Figure 8).
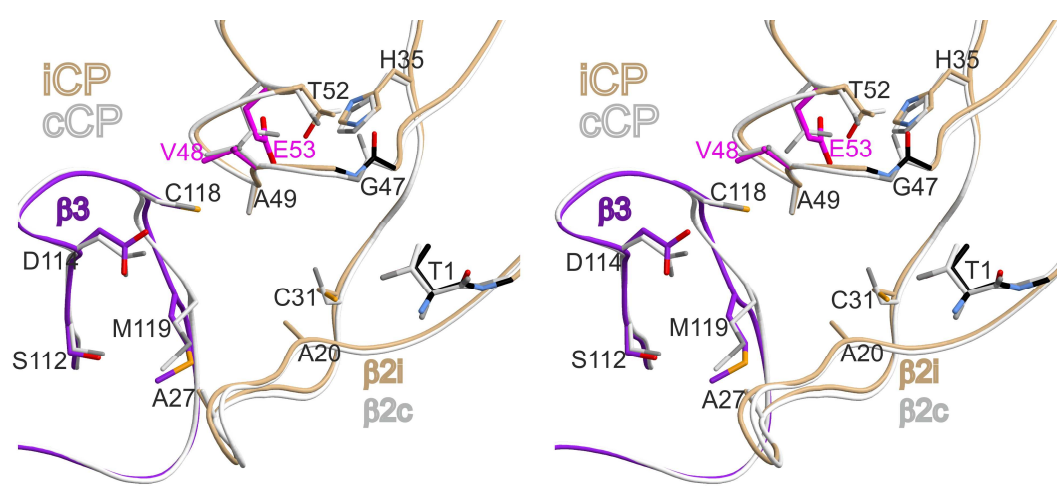

Figure 14 Superposition of the unprimed $\beta 2 \mathrm{c}$ and $\beta 2 \mathrm{i}$ substrate binding channels (Stereo view). Amino acid numbers are given for subunit $\beta 2 \mathrm{i}$ and residues characteristic of $\beta 2 \mathrm{i}$ are highlighted in magenta. Adapted from Huber et al., 2012 ${ }^{[32]}$.

By contrast, the $\beta 1 \mathrm{c}$ and $\beta 1 \mathrm{i}$ subunits strongly differ in their amino acid lining in the unprimed substrate binding channel. The polar active site surrounding of subunit $\beta 1 \mathrm{c}$ is replaced by a more hydrophobic one in subunit $\beta 1$ i. In particular the amino acid substitutions T20V, T31F, $\mathrm{R} 45 \mathrm{~L}$ and T52A decrease the polarity and the size of the $\beta 1 \mathrm{i}$ S1 pocket (Figure 15). Hence, the $\beta 1 \mathrm{i}$ active site preferentially cleaves proteins $\mathrm{C}$-terminally of small hydrophobic and branched amino acids such as Leu, Ile or Val and significantly enhances the production of high-affinity MHC I ligands. The structural features of subunit $\beta 1 \mathrm{i}$ are in full agreement with the reported $\beta 1$ i-selective fluorogenic substrate Ac-Pro-Ala-Leu-AMC ${ }^{[110]}$. In addition to the changes in the $\mathrm{S} 1$ pocket, the $\mathrm{S} 3$ pocket is characterized by the amino acid substitutions $\mathrm{T} 22 \mathrm{~A}$ $(\beta 1 \mathrm{i})$ and $\mathrm{A} 27 \mathrm{~V}(\beta 1 \mathrm{i})$ as well as $\mathrm{Y} 114 \mathrm{H}$ in the neighbouring subunit $\beta 2 \mathrm{i}$. These differences lead to a more size-restricted and more polar S3 pocket in the iCP (Figure 15).
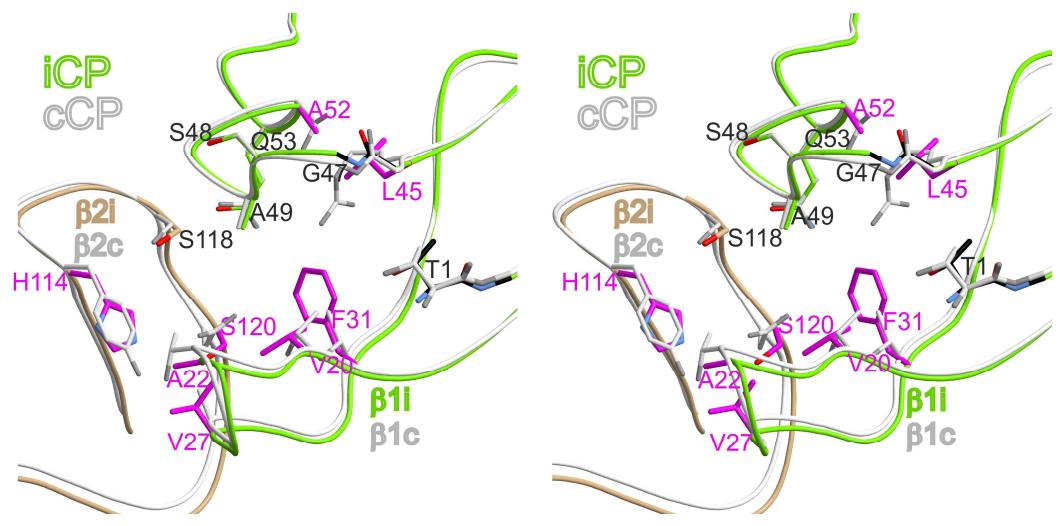

Figure 15 Superposition of the unprimed $\beta 1 \mathrm{c}$ and $\beta 1 \mathrm{i}$ substrate binding channels (Stereo view). Amino acid numbers are given for subunit $\beta 1 \mathrm{i}$ and residues characteristic of $\beta 1 \mathrm{i}$ are highlighted in magenta. Adapted from Huber et al., 2012 $2^{[32]}$. 
The hydrophobic amino acid lining of the $S 1$ pocket in subunit $\beta 5 \mathrm{c}$ is preserved in subunit $\beta 5 \mathrm{i}$ with respect to the amino acids Ala20, Met45, Ala49 and Cys52 (Figure 16). The amino acid side chain 31 is also hydrophobic but variable in length (e.g. Met in mice and Val in humans). Thus, both subunits exert overlapping substrate specificities and hydrolyse proteins after apolar residues. Interestingly, all known $\beta 5 \mathrm{i}$ subunits possess a shallow $\mathrm{S} 2$ pocket formed by either Cys48 or Ser48 (Figure 16). The incorporation of Ser27 in the S3 pockets of murine and human $\beta 5$ i subunits reduces their size but concomitantly increases their polarity.
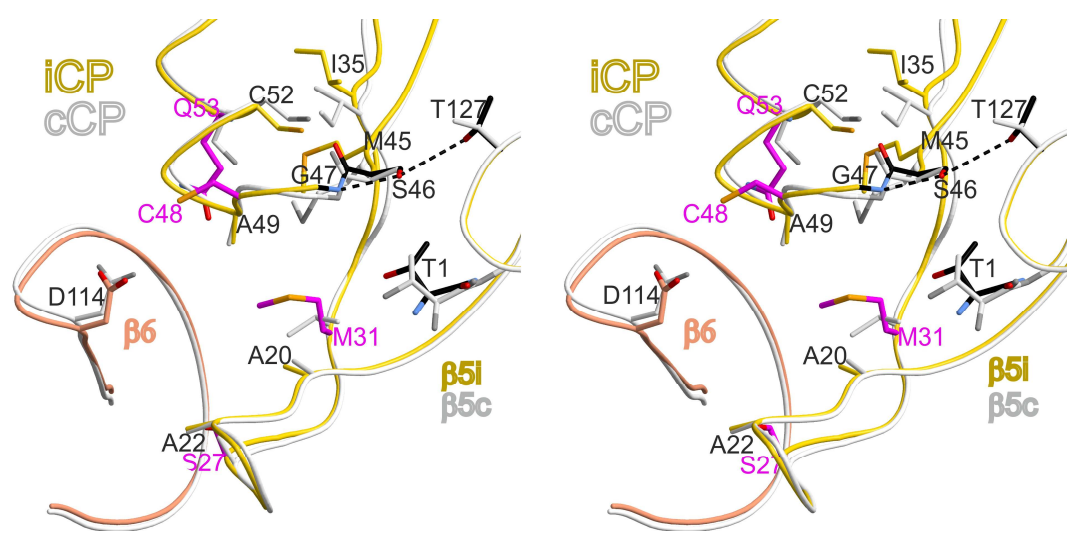

Figure 16 Superposition of the unprimed $\beta 5 \mathrm{c}$ and $\beta 5 \mathrm{i}$ substrate binding channels (Stereo view). Amino acid numbers are given for subunit $\beta 5 \mathrm{i}$ and residues characteristic of the $\beta 5 \mathrm{i}$ substrate binding pockets are highlighted in magenta. Being hydrogen-bridged to the oxyanion hole Gly47NH and to Thr127 (black dashed lines), Ser46 is supposed to influence the catalytic activity of subunit $\beta 5 \mathrm{i}$. Note that the side chain conformations of Ile 35 and Met45 vary in the subunits $\beta 5 \mathrm{c}$ and $\beta 5 \mathrm{i}$.

Adapted from Huber et al., 2012 $2^{[32]}$.

A striking feature of the $\beta 5 \mathrm{i}$ subunit is the increased size of its $\mathrm{S} 1$ pocket compared to subunit $\beta 5 \mathrm{c}$. This is mostly due to distinct conformations of Met 45 and Ile 35 in subunit $\beta 5 \mathrm{i}$ as well as a shift of the protein backbone including the residues 36 to 76 compared to subunit $\beta 5 \mathrm{c}$ (Figure 16). In this regard, the amino acid exchange S53Q between subunit $\beta 5 \mathrm{c}$ and $\beta 5 \mathrm{i}$ might have a pivotal function. In subunit $\beta 5 i$ the aliphatic part of the Gln53 side chain can stabilize Met45, while in subunit $\beta 5 \mathrm{c}$ Ser53 cannot provide these favourable interactions and thus, the $\mathrm{S} 1$ pocket is significantly smaller than that of its i counterpart (see also section 6.4.1). The differently-sized S1 pockets are supposed to give rise to varying substrate specificities. Although both subunits $\beta 5 \mathrm{c}$ and $\beta 5 \mathrm{i}$ accommodate hydrophobic amino acids in their S1 pockets, the $\beta 5$ i subunit prefers more spacious ones than subunit $\beta 5 c^{[110]}$ (see also chapter 6.3.3).

Moreover, the active site Thrl of subunit $\beta 5 \mathrm{i}$ is surrounded by a unique hydrophilicity that results from the amino acid exchanges A46S and V127T and that is not observed in any other proteasome subunit. Ser46O $\mathrm{O}^{\gamma}$ is in hydrogen bonding distance to $\mathrm{T}_{12} 2 \mathrm{O}^{\gamma}$ and the oxyanion 
hole Gly47NH (distances 2.9-3.4 $\AA$; Figure 16). This unique hydrogen bond network might stabilize the tetrahedral transition state during catalysis. Additionally, it could kinetically favour the $\beta 5 \mathrm{i}$ active site, as the increased polar environment is supposed to attract water molecules and thereby to enhance peptide bond hydrolysis ${ }^{[32]}$. 


\subsection{Investigations on the $\beta 5$ i-selective proteasome inhibitor ONX 0914}

\subsubsection{Inhibition of $20 \mathrm{~S}$ proteasomes by ONX 0914}

The $\alpha^{\prime}, \beta$ 'epoxyketone inhibitor ONX 0914 has been shown to selectively inhibit the $\beta 5 \mathrm{i}$ subunit of the murine and human $\mathrm{iCP}$ and to be therapeutically active in inflammatory disorders and autoimmune diseases ${ }^{[37,53]}$. To verify these results and to further assess the purities of the murine $\mathrm{cCP}$ and $\mathrm{iCP}$ preparations, $\mathrm{IC}_{50}$ values of $\mathrm{ONX} 0914$ were determined for the ChTL activity of the yCP, the murine $\mathrm{CCP}$ and $\mathrm{iCP}$ as well as the human $\mathrm{CCP}$ and iCP (Figure 17). The activities of CPs were measured in the presence of varying concentrations of ONX 0914, using the fluorogenic substrate Suc-Leu-Leu-Val-Tyr-AMC. In agreement with previous studies, the $\beta 5 \mathrm{c}$ subunits from mouse and human cCPs were far less inhibited than their i counterparts (Figure 17). Interestingly, however, ONX 0914 slightly favoured subunit y $\beta 5$ over $\beta 5 \mathrm{c}$.

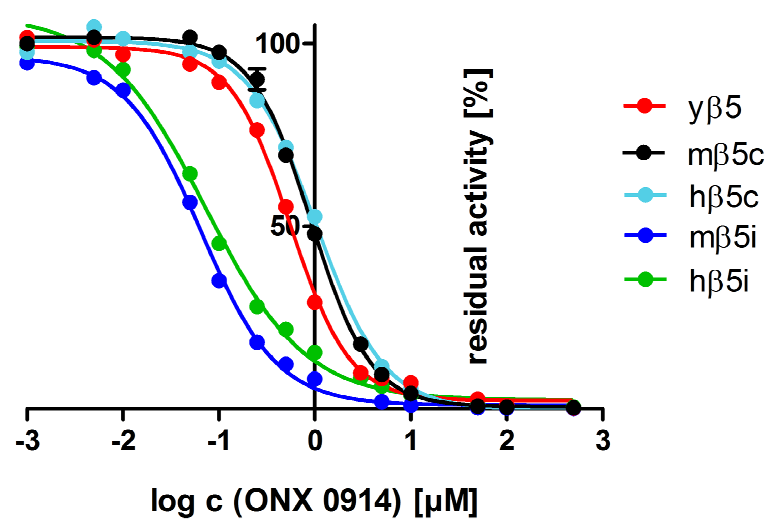

\begin{tabular}{ll}
\hline $\boldsymbol{\beta 5}$-subunit & $\boldsymbol{I C}_{\mathbf{5 0}}[\boldsymbol{\mu M}]$ \\
\hline $\mathrm{y} \beta 5$ & $0.551 \pm 0.035$ \\
$\mathrm{~m} \beta 5 \mathrm{c}$ & $0.917 \pm 0.054$ \\
$\mathrm{~h} \beta 5 \mathrm{c}$ & $1.036 \pm 0.054$ \\
$\mathrm{~m} \beta 5 \mathrm{i}$ & $0.065 \pm 0.005$ \\
$\mathrm{~h} \beta 5 \mathrm{i}$ & $0.073 \pm 0.011$ \\
\hline
\end{tabular}

Figure 17 Selectivity of ONX 0914 for the $\beta 5 i$ subunit of the iCP. After the exposure of yCP, $\mathrm{cCP}$ and iCP to varying concentrations of ONX $0914(0.001-500 \mu \mathrm{M})$ the residual ChTL activity was determined. Data from three experiments were normalized to DMSO treated controls and averaged. Standard deviations are indicated. $\mathrm{IC}_{50}$ values were deduced from fitted data.

Adapted from Huber et al., 2012 $2^{[32]}$.

\subsubsection{Proteasome core particles in complex with ONX 0914}

Structural explanations for the $\beta 5$ i selectivity of ONX 0914 could only be unravelled by analysing and comparing ligand binding to each active site of the $\mathrm{cCP} / \mathrm{yCP}$ and $\mathrm{iCP}$. Inhibitor soaking experiments with a final concentration of ONX 0914 of $3 \mathrm{mM}$ blocked all proteolytic centres - not only the preferred subunit $\beta 5 \mathrm{i} .2 \mathrm{~F}_{\mathrm{O}}-\mathrm{F}_{\mathrm{C}}$ electron density maps depicted that ONX 0914 was covalently bound to all active sites of cCP, iCP and yCP (Figure 18) and additionally proved that these were catalytically active in the crystals. Despite suggestions 

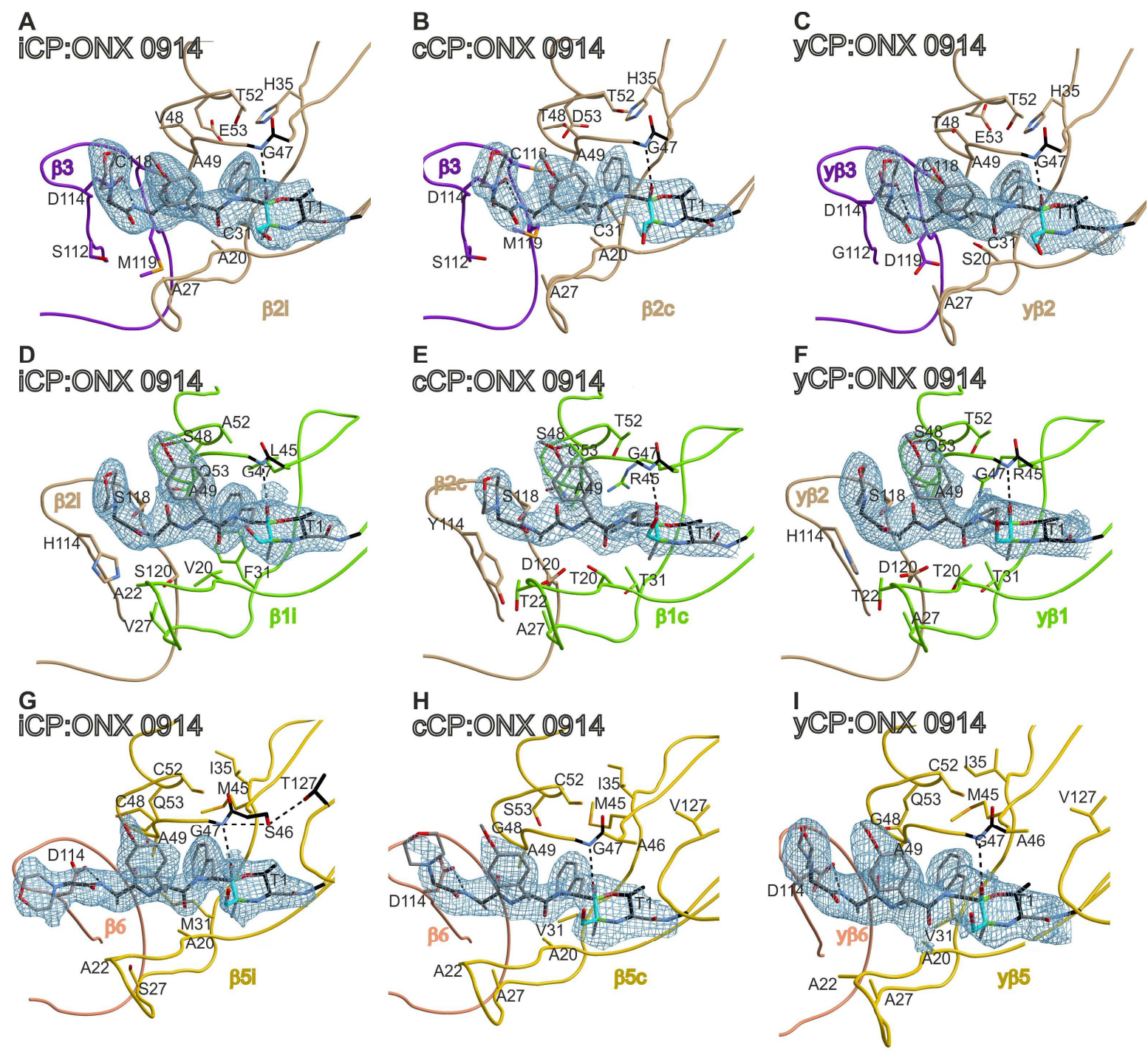

Figure 18 ONX 0914 bound to the proteolytically active sites of yCP, cCP and iCP. The $2 \mathrm{~F}_{\mathrm{O}}-\mathrm{F}_{\mathrm{C}}$ electron density maps, contoured to $1 \sigma$, show full occupancy for ONX 0914 at all active centres of yCP, cCP and iCP. ONX 0914 and residue Thr1 have been omitted for phasing. Hydrogen bonds are marked by black dashed lines. Adapted from Huber et al., 2012 ${ }^{[32]}$.

that subunit $\beta 7$ might also exert hydrolytic activity in the ligand-free bovine $\mathrm{cCP}$ crystal structure $^{[21 a]}$, ONX 0914 was not bound to this subunit.

Likewise other peptidic CP inhibitors, the C-terminal dipeptide of ONX 0914 forms an antiparallel $\beta$ sheet in the unprimed substrate binding channels of all active sites and is well stabilized by favourable interactions with the surrounding protein residues. In contrast, the Nterminal moropholine moiety of ONX 0914 is not engaged in any contact with the substrate binding channels and thus, can adopt different conformations. Unique to all $\beta 2$ and $\beta 5$ subunits is the additional stabilization of ONX 0914 via a hydrogen bond between its Nterminal peptide bond and Asp114 from the neighbouring subunits $\beta 3$ and $\beta 6$, respectively. Furthermore, structural comparison of all ONX 0914 molecules bound to the yCP, cCP and iCP proves a similar mechanism of inhibitor binding for all active sites (Figure 19). 
A

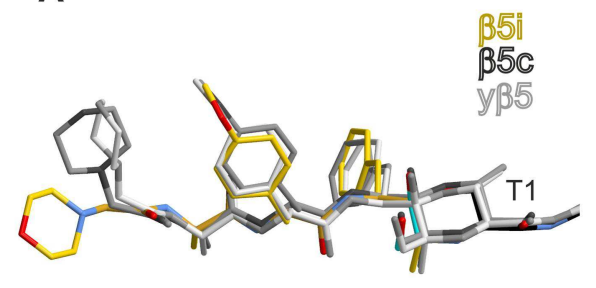

B

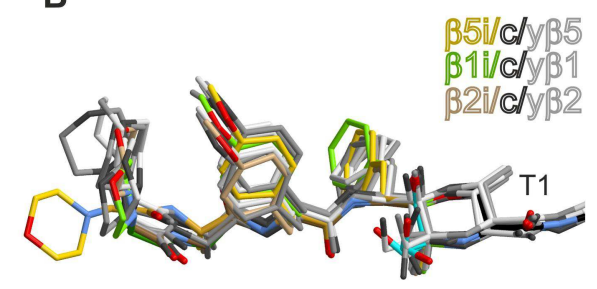

Figure 19 Superposition of ONX 0914 molecules bound to the yCP, cCP and iCP. (A) The binding mechanism of ONX 0914 to Thr1 is identical for all $\beta 5$ active sites and (B) for all proteasome subunits. The peptidic ligand adopts an anti-parallel $\beta$ sheet in each substrate binding channel, thereby mimicking natural protein substrates.

\subsubsection{Molecular basis for the subunit selectivity of ONX 0914}

Comparison of active proteasome subunits in the presence and absence of ONX 0914 visualized the structural features that cause the observed $\beta 5$ i selectivity of this compound. Owing to Ala20, Ala27, Cys31 and Gly45 the $\beta 2$ subunits possess large S1 pockets that can easily accommodate ONX 0914 without any structural changes (Figure 20A-C).

A

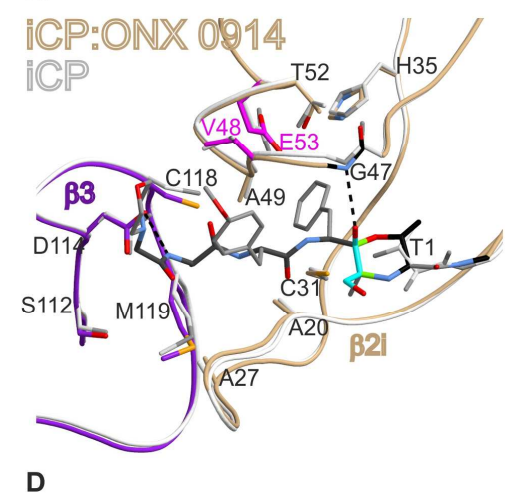

ICP:ONX 0914:

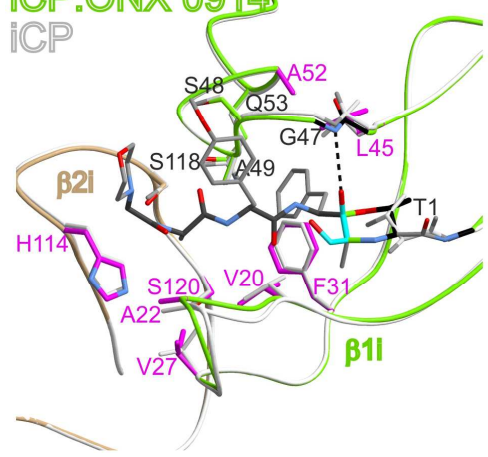

B

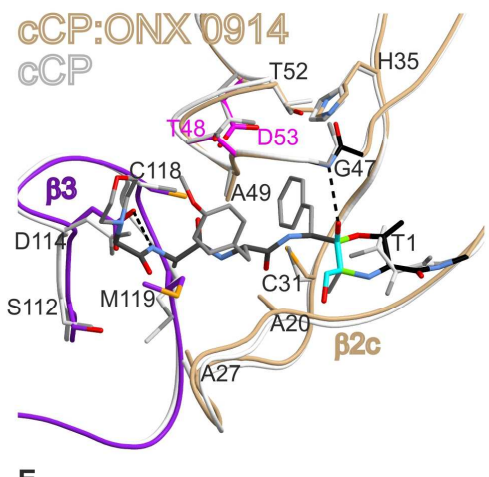

E

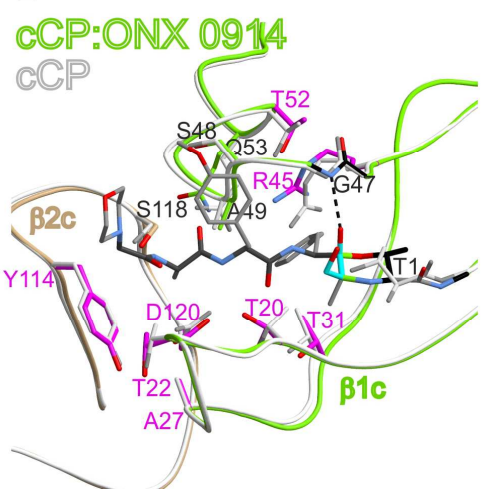

C
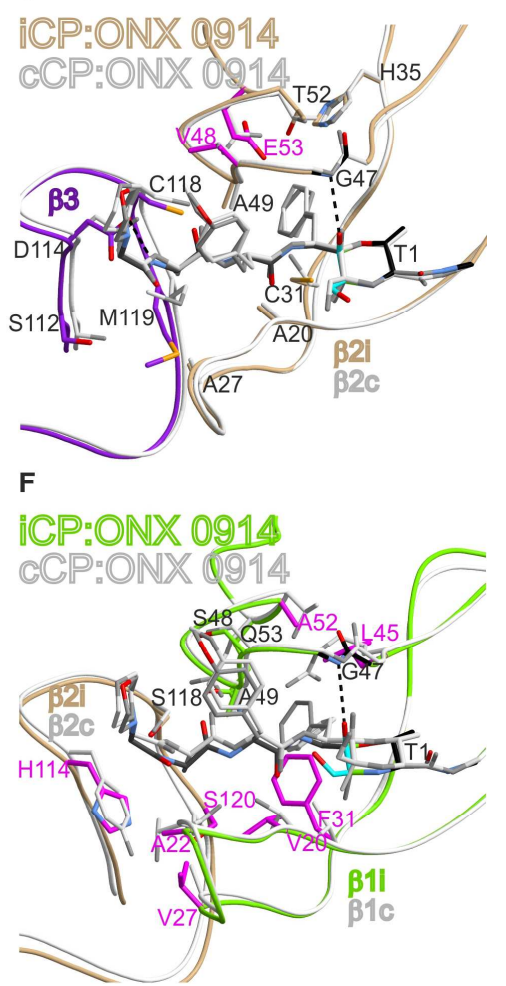

Figure 20 Superposition of ligand-free and ligand-bound active sites of the proteasome. The unliganded subunits $\beta 2 \mathrm{i}(\mathbf{A}), \beta 2 \mathrm{c}(\mathbf{B}), \beta 1 \mathrm{i}(\mathbf{D})$ and $\beta 1 \mathrm{c}(\mathbf{E})$ are superimposed to their ONX 0914 bound states. The panels $(\mathbf{C})$ and $(\mathbf{F})$ illustrate the differences for the ligand-bound $\beta 2 \mathrm{c} / \mathrm{i}$ and $\beta 1 \mathrm{c} / \mathrm{i}$ subunits, respectively; amino acid numbers are given for the $\mathrm{i}$ subunit. Residues characteristic of the $\mathrm{i}$ or $\mathrm{c}$ subunits are highlighted in magenta. Hydrogen bonds are indicated by black dashed lines.

Adapted from Huber et al., 2012 ${ }^{[32]}$. 
However, as the protein surrounding provides only few stabilizing interactions, ligand binding to the $\beta 2 \mathrm{c}$ and $\beta 2 \mathrm{i}$ active sites is energetically disadvantaged compared to subunit $\beta 5 \mathrm{i}$.

By contrast, binding of ONX 0914 triggers structural rearrangements in subunit $\beta 1 \mathrm{c}$. Due to steric hindrance with the ligand's phenylgroup in P1, Arg45 has to change its conformation (Figure 20E). Moreover, the polarity of the S1 pocket disfavours the binding of the hydrophobic P1 side chain of ONX 0914 to subunit $\beta 1 \mathrm{c}$ and causes electrostatic repulsion.

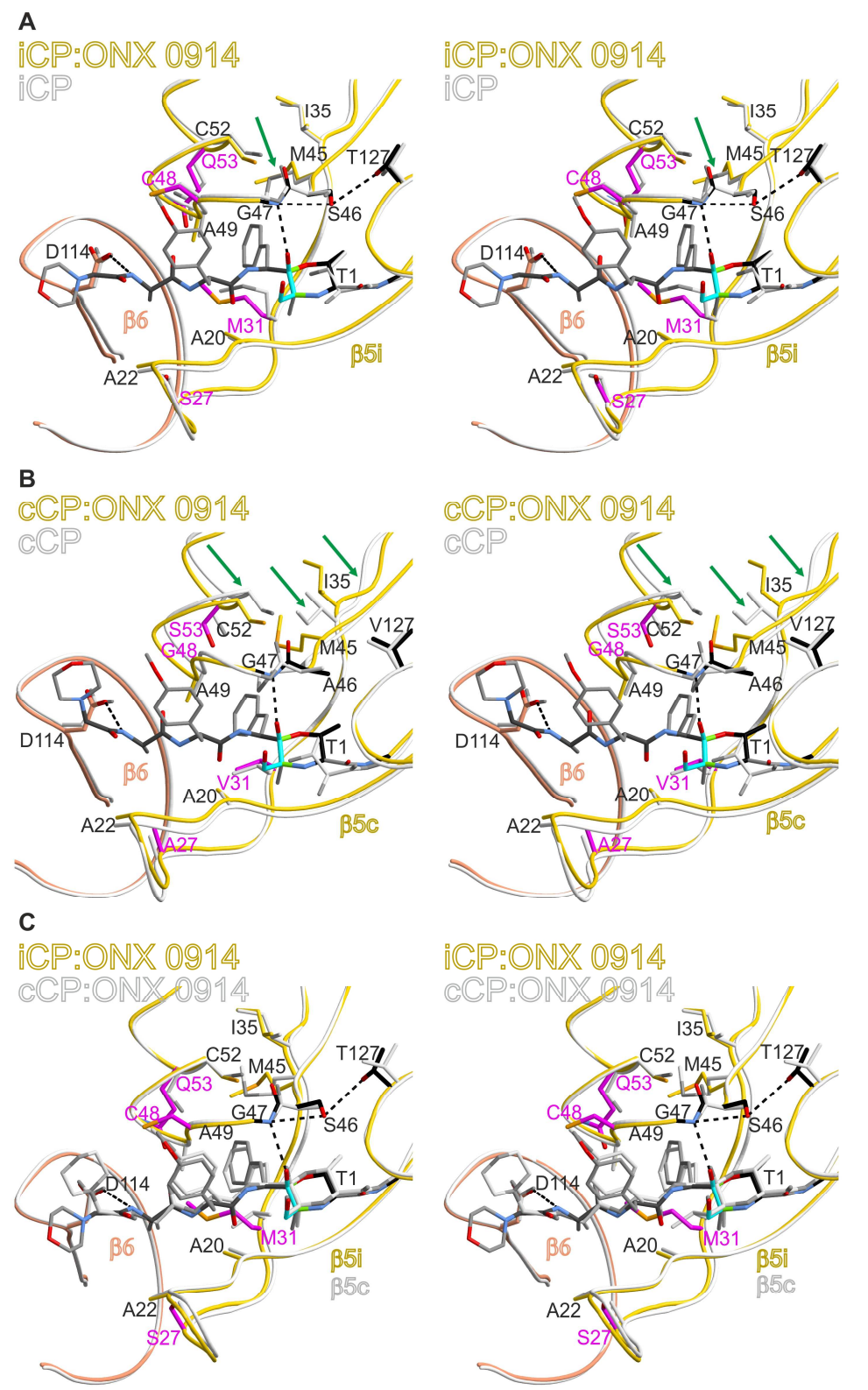

Figure 21 Binding of ONX 0914 to the $\beta 5 c$ and $\beta 5$ i subunits of the cCP and iCP (Stereo view). (A) The unprimed substrate binding channel of subunit $\beta 5 \mathrm{i}$ is ideally suited to accommodate ligands with bulky P1 side chains such as ONX 0914. Upon binding neighbouring protein residues only slightly rearrange (green arrow). (B) Docking of ONX 0914 to subunit $\beta 5$ c triggers major structural changes around the active site as marked by green arrows. (C) The $\beta 5 \mathrm{c}$ and $\beta 5 \mathrm{i}$ subunits in their ligand bound states are structurally similar to each other. Adapted from Huber et al., 2012 $2^{[32]}$. 
Therefore the hydrophobic substrate binding channel of the $\beta 1 \mathrm{i}$ active site should be ideally suited for docking of ONX 0914. Yet, the ligand complex structure indicates that Phe31 sterically hinders binding of ONX 0914 to Thr1 (Figure 20D, F). The distance between the phenyl group of Phe31 and the P1 phenylalanine of ONX 0914 is only 3.4-3.5 $\AA$ and Phe31 adopts an energetically disfavoured orientation towards the carbonyl oxygen of Asp32 (distance $3.2 \AA$ ). Thus, the atomic distances reflect the repelling forces that prevent highaffinity binding of ONX 0914 to subunit $\beta 1$ i.

Ligand binding to the $\beta 5 \mathrm{i}$ subunit triggers slight conformational adaptions of Met31 and the $\mathrm{CH}_{3}$-S-group of Met45 (r.m.s.d. $\mathrm{C}_{\alpha} \beta 5 \mathrm{i} / \beta 5 \mathrm{i}$ :ONX 0914: $0.28 \AA$; Figure $21 \mathrm{~A}$ ). By contrast, the comparison of the unprimed substrate binding channel of subunit $\beta 5 \mathrm{c}$ in the presence and absence of ONX 0914 reveals profound differences. Binding of the bulky P1 phenylalanine of the $\alpha$, $\beta$ ' epoxyketone to the $\mathrm{S} 1$ pocket displaces Met45 from its normal position. Furthermore, the side chain of Ile35 is flipped and the $\beta$ sheets S4 and S5 as well as $\alpha$ helix H1 are offset by
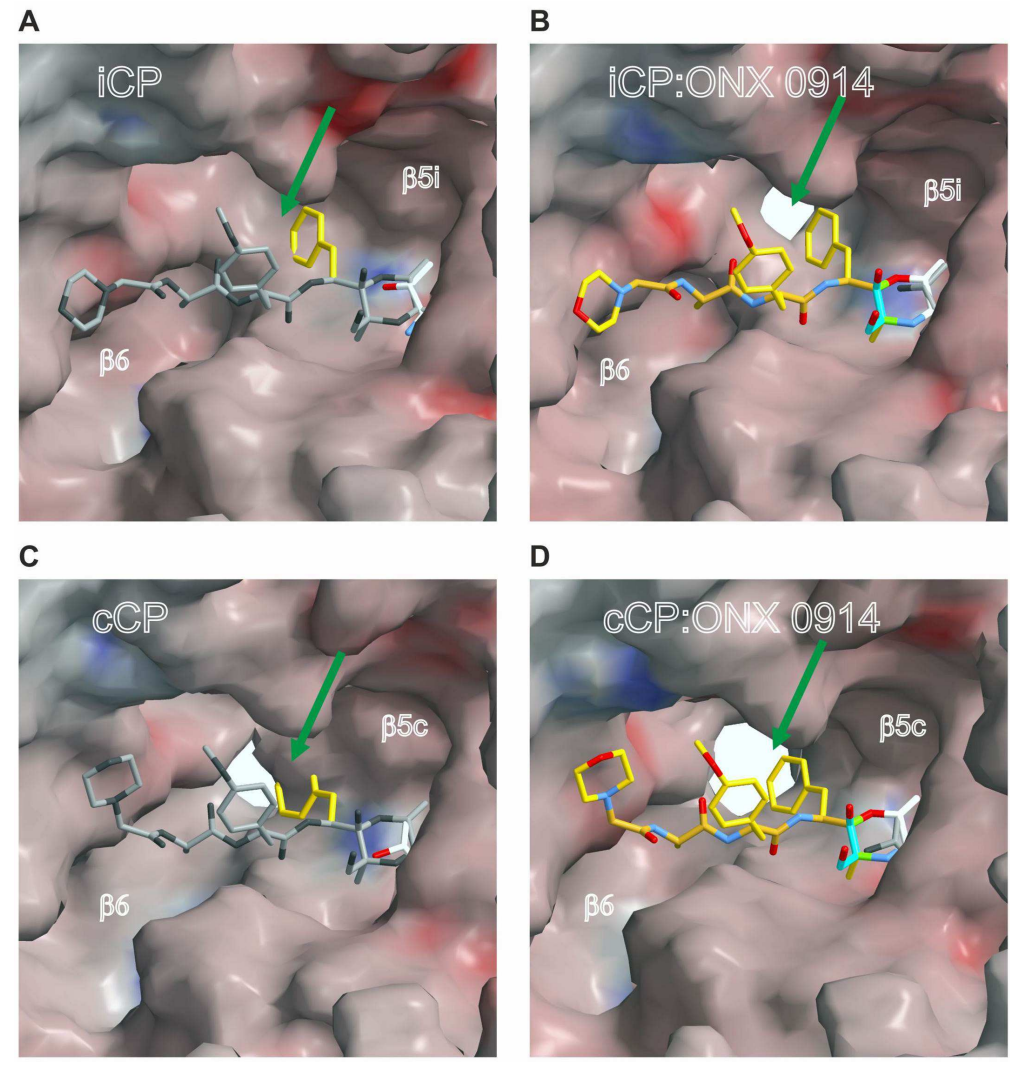

Figure 22 Conolly surface representations of the unprimed $\beta 5 \mathrm{c}$ and $\beta 5 \mathrm{i}$ substrate binding channels in the presence and absence of ONX 0914. Surface charge distributions are shown for the subunits $\beta 5$ i (A), $\beta 5 \mathrm{i}: O N X 0914$ (B), $\beta 5 \mathrm{c}(\mathbf{C})$ and $\beta 5 \mathrm{c}: O N X 0914$ (D) with positive and negative electrostatic potentials contoured from $50 \mathrm{kT} / \mathrm{e}$ (intense blue) to $-50 \mathrm{kT} / \mathrm{e}$ (intense red). In the panels (A) and (C) ONX 0914 has been modelled into the active site by superposition with the panels $(\mathbf{B})$ and $(\mathbf{D})$, respectively. The catalytic Thr1 is coloured in white. ONX 0914 is shown in yellow, modelled molecules of ONX 0914 in grey - except for the P1 site. To depict ligand-protein clashes and resulting structural changes (green arrows) the amino acids 46-50 of the subunits $\beta 5 \mathrm{c}$ and $\beta 5 \mathrm{i}$ were removed. Adapted from Huber et al., 2012 $2^{[32]}$. 
up to $1.7 \AA$ (r.m.s.d. $\mathrm{C}_{\alpha} \beta 5 \mathrm{c} / \beta 5 \mathrm{c}$ :ONX 0914: $0.64 \AA$; Figure $21 \mathrm{~B}$ ). Remarkably, the ligand bound states of the subunits $\beta 5 \mathrm{c}$ and $\beta 5 \mathrm{i}$ are similar to each other (r.m.s.d $\mathrm{C}_{\alpha} \beta 5 \mathrm{c}$ : ONX 0914/ß5i:ONX 0914: $0.55 \AA$; Figure 21C) and the $\beta 5 \mathrm{c}: O N X 0914$ structure highly resembles the apostructure of subunit $\beta 5 \mathrm{i}$. These results indicate that inhibition of $\beta 5 \mathrm{c}$ by ONX 0914 is sterically impaired by Met45. The enthalpic energy required to achieve the observed structural changes is mirrored in the high $\mathrm{IC}_{50}$ value of ONX 0914 towards $\beta 5 \mathrm{c}$ compared to $\beta 5 \mathrm{i}$ and its lower affinity. Conolly surface representations of the subunits $\beta 5 \mathrm{c}$ and $\beta 5 \mathrm{i}$ clearly depict the structural differences in their S1 pocket architecture. Whereas subunit $\beta 5 \mathrm{i}$ possesses a spacious $\mathrm{S} 1$ site, which enables the binding of bulky P1 residues (Figure 22A) with only minor side chain rearrangements (Figure 22B), the S1 pocket forming residues of subunit $\beta 5 c$ severely clash with bulky P1 groups (Figure 22C) and thereby cause enormous structural reorientations (Figure 22D). Hence, the substrate specificity of subunit $\beta 5 \mathrm{i}$ can be described as a ChTL activity, whereas the $\beta 5 \mathrm{c}$ active site preferentially exerts elastase-like or Snaap activity ${ }^{[33 b]}$.

\subsubsection{Structural analysis of the epoxyketone reaction mechanism}

Whereas boronic acid inhibitors are known to inhibit serine and threonine proteases ${ }^{[68]}$, $\alpha^{\prime}, \beta$ 'epoxyketones exclusively react with the small family of Ntn hydrolases to which the proteasome belongs. This high degree of specificity for the CP results from the unique bivalent reaction mechanism of $\alpha^{\prime}, \beta$ 'epoxyketones, which has been elucidated for the natural product epoxomicin isolated from actinomycetes ${ }^{[61 \mathrm{a}]}$ : The nucleophilic $\mathrm{Thr} 1 \mathrm{O}^{\gamma}$ of the active proteasome subunit attacks the carbonyl carbon atom of the $\alpha^{\prime}, \beta$ 'epoxyketone, thereby

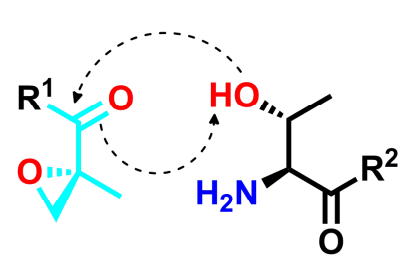

$\alpha^{\prime}, \beta^{\prime}$ Epoxyketone Thr1 of active $\beta$ subunits

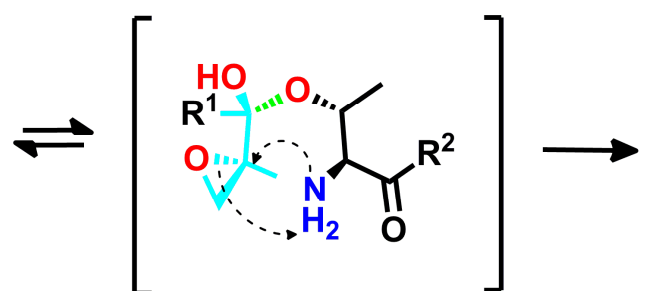

Hemiketal<smiles>[R]C(=O)C1N[C@]2(CO)[C@@H](C)O[C@H](C)[C@]2([R2])O1</smiles>

Morpholine ring / Secondary amine

Figure 23 Schematic illustration of the reaction mechanism of epoxyketone inhibitors. Thr10 ${ }^{\gamma}$ nucleophilically attacks the carbonyl carbon atom of the epoxyketone inhibitor. The formed hemiketal intermediate can either dissociate to deliberate $\mathrm{Thr}_{10} \mathrm{O}^{\gamma}$ or irreversibly cycle under the formation of a secondary amine (morpholine ring system) involving Thr1N. Bonds created by this reaction are coloured in green, $\mathrm{R}_{1}$ corresponds to the peptide moiety of the compound, $R_{2}$ to the proteolytically active $\beta$ subunit, whose Thr1 is covalently modified by the displayed reaction mechanism. 


\section{Crystal parameters}

Space group

Cell constants

$\mathrm{CPs} / \mathrm{AU}^{\mathrm{a}}$

\section{Data collection}

\section{Beam line}

Wavelength $(\AA)$

Resolution range $(\AA)^{\mathrm{b}}$

No. observations

No. unique reflections ${ }^{\mathrm{c}}$

Completeness (\%) ${ }^{\mathrm{b}}$

$\mathrm{R}_{\text {merge }}(\%)^{\mathrm{b}, \mathrm{d}}$

$\mathrm{I} / \sigma(\mathrm{I})^{\mathrm{b}}$

\section{Refinement (REFMAC5)}

Resolution range $(\AA)$

No. refl. working set

No. refl. test set

No. non hydrogen

No. of ligand atoms

Water molecules

$\mathrm{R}_{\text {work }} / \mathrm{R}_{\text {free }}(\%)^{\mathrm{e}}$

r.m.s.d. bond $(\AA) /\left({ }^{\circ}\right)^{\mathrm{f}}$

Average B-factor $\left(\AA^{2}\right)$

Ramachandran Plot $(\%)^{\mathrm{g}}$

$\begin{array}{ll}\mathrm{P} 2_{1} & \mathrm{P} 2_{1} \\ \mathrm{a}=135.4 \AA & \mathrm{a}=134.4 \AA \\ \mathrm{b}=300.4 \AA & \mathrm{b}=300.8 \AA \\ \mathrm{c}=143.9 \AA & \mathrm{c}=143.8 \AA \\ \beta=112.8^{\circ} & \beta=112.8^{\circ} \\ 1 & 1\end{array}$

\section{X06SA, SLS}

1.0

$30-2.7$

(2.8-2.7)

1211656

286910

$99.1(98.7)$

$9.8(59.6)$

$11.3(2.7)$

X06SA, SLS

1.0

30-3.4

$(3.5-3.4)$

443424

141633

$98.1(98.3)$

$14.3(59.6)$

$8.2(2.2)$

$\begin{array}{ll}15-2.7 & 15-3.4 \\ 272564 & 134550 \\ 13628 & 6727 \\ 50924 & 51122 \\ 36 & 294 \\ 1340 & 1322 \\ 22.3 / 24.3 & 17.9 / 22.0 \\ 0.005 / 0.834 & 0.005 / 0.899 \\ 54.6 & 80.5 \\ 97.3 / 2.4 / 0.3 & 96.3 / 3.2 / 0.5\end{array}$

Table 13 X-ray data collection and refinement statistics of yCP structures in complex with ONX 0914.

a Asymmetric unit.

b The values in parentheses of resolution range, completeness, $R_{\text {merge }}$ and $I / \sigma$ (I) correspond to the last resolution shell.

c Friedel pairs were treated as identical reflections.

${ }^{\mathrm{d}} \mathrm{R}_{\text {merge }}(\mathrm{I})=\Sigma_{\mathrm{hkl}} \Sigma_{\mathrm{j}}\left|\left[\mathrm{I}(h k l)_{\mathrm{j}}-\mathrm{I}(h k l)\right]\right| \Sigma_{\mathrm{hkl}} \mathrm{I}_{\mathrm{hkl}}$, where $\mathrm{I}(\mathrm{hkl})_{\mathrm{j}}$ is the $\mathrm{j}^{\text {th }}$ measurement of the intensity of reflection hkl and $\langle\mathrm{I}(\mathrm{hkl})\rangle$ is the average intensity.

e $\mathrm{R}=\Sigma_{\mathrm{hkl}}|| \mathrm{F}_{\mathrm{obs}}|-| \mathrm{F}_{\text {calc }}|| \Sigma_{\text {hkl }}\left|\mathrm{F}_{\mathrm{obs}}\right|$, where $\mathrm{R}_{\text {free }}$ is calculated for a randomly chosen $5 \%$ of reflections, which were not used for structure refinement, and $\mathrm{R}_{\mathrm{work}}$ is calculated for the remaining reflections.

$\mathrm{f}$ Deviations from ideal bond lengths/angles.

$\mathrm{g}$ Number of residues in favoured, allowed or outlier region.

forming a reversible hemiketal. Subsequently, the free N-terminus of Thr1 opens the epoxide ring and, by an irreversible cyclisation, creates a morpholine ring system (Figure 23). This mode of action and the docking mechanism of ONX 0914 was investigated in detail by ONX 0914:yCP complex structures. The yCP can be used as a model system due to its structural similarity to the cCP, including the orientation of Met45 (Figure 26A). yCP crystals were therefore soaked with ONX 0914 in different concentrations for varying time periods. Hereby, the reaction intermediate - the hemiketal with an intact epoxide (ep) - and the reaction product - the morpholine ring (mo) - could be trapped in a crystal structure (Table 
13). These data clarified that indeed Thr1N attacks the epoxide once the hemiketal is formed, and proved the formerly proposed mode of action of epoxyketone inhibitors ${ }^{\text {[61a] }}$.

Whereas all three proteolytically active sites were covalently modified in the yCP:ONX 0914_mo structure, only subunit y 35 visualized the hemiketal formation of ONX 0914 (Figure 24A). In agreement with data on the murine and human $\mathrm{CCP}^{[37]}$, ONX 0914 favours subunit y $\beta 5$ over $y \beta 1$ and $y \beta 2$.

Notably, the $2 \mathrm{~F}_{\mathrm{O}}-\mathrm{F}_{\mathrm{C}}$ electron density map for the yCP:ONX 0914_mo structure displayed the whole inhibitor with its $\mathrm{P} 1, \mathrm{P} 2$ and $\mathrm{P} 3$ residues as well as the $\mathrm{N}$-terminal morpholine ring and the covalent linkage to Thr1 (Figure 24B). In contrast, the electron density map for the hemiketal intermediate (yCP:ONX 0914_ep) surprisingly depicted solely the intact epoxide ring and the ligand's P1 site (Figure 24A). As the N-terminal P3 and P2 sites of ONX 0914_ep were not defined, they were not yet bound to their respective substrate specificity pockets and thus, flexible. This finding provides evidence that first the electrophilic warhead and the P1 residue of covalently acting inhibitors contact the active site
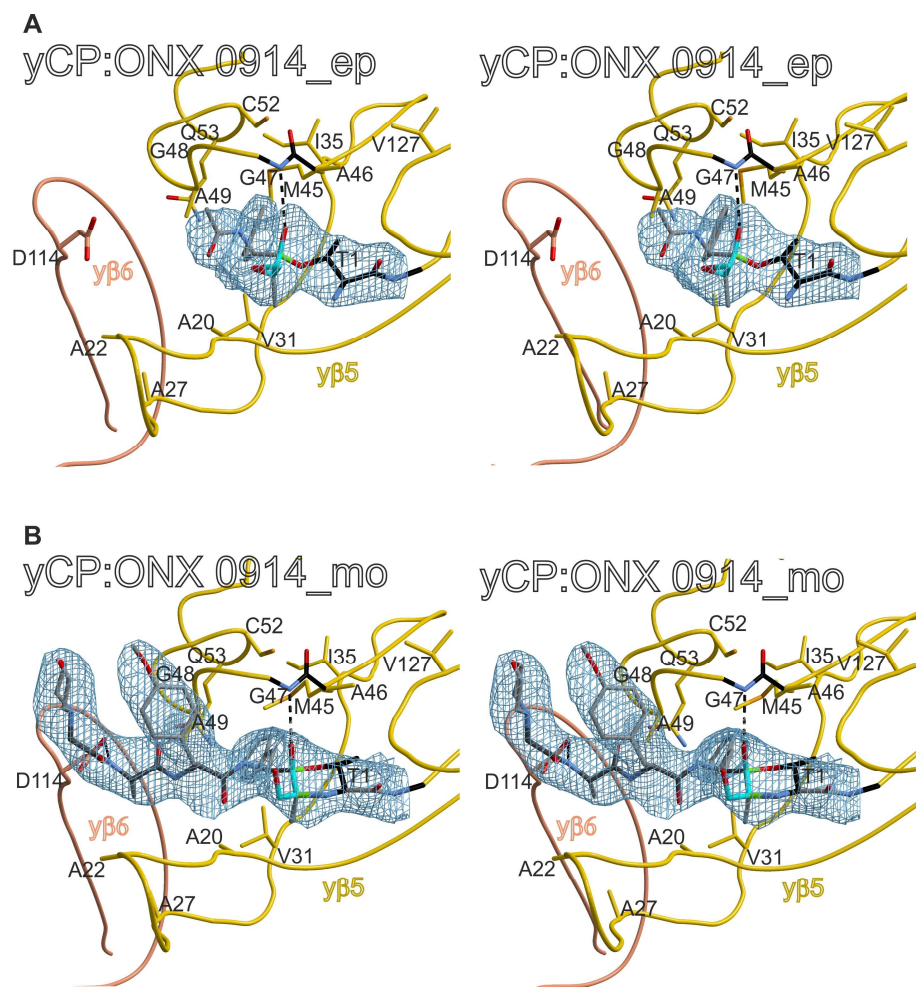

Figure 24 Stereo view of the yeast $20 S$ proteasome subunit $\beta 5$ (yß5) in complex with ONX 0914. Subunit y $\beta 5$ is coloured in yellow and the neighbouring subunit y $\beta 6$ in orange. Thr1 and the oxyanion hole Gly47NH are marked in black; and hydrogen bonds are indicated by black dashed lines. ONX 0914 is shown in grey with its reactive head group highlighted in cyan. The experimental $2 \mathrm{~F}_{\mathrm{O}}-\mathrm{F}_{\mathrm{C}}$ omit electron density map (blue mask) is contoured to $1 \sigma$. (A) Hemiketal formation of ONX 0914 with Thr1 of subunit y $\beta 5$. Note, only the epoxide and the P1 site of ONX 914 are well-defined in the electron density map. (B) Subunit y 35 in complex with the completely reacted ONX 0914. All inhibitor side chains (P1, P2 and P3) are visualized in the electron density. Adapted from Huber et al., 2012 $2^{[32]}$. 
Thr1 and the S1 pocket and that only upon completing the reaction mechanism interactions with the S2 and S3 sites form the antiparallel $\beta$ sheet in the substrate binding channel. In conclusion, even though all substrate binding pockets contribute to the $\mathrm{IC}_{50}$ value of a compound, the P1 side chain of a compound, apart from its electrophilic pharmacophore, is mostly responsible for its affinity for the active site. In this regard the depicted size differences of the $\beta 5 \mathrm{c}$ and $\beta 5 \mathrm{i} S 1$ pockets provoke the enhanced affinity of ONX 0914 for subunit $\beta 5$ i.

Structural comparison of the hemiketal intermediate and the final reaction product reveals that although the P1 residue of the ONX 0914_ep is defined in the electron density map, it initially does not fully occupy the $\mathrm{S} 1$ pocket of subunit y $\beta 5$ (Figure 25). Hence, during the reversible hemiketal formation no sterical clashes with Met45 occur and in agreement with the X-ray structure of the intermediate state no displacement of Met45 is observed (Figure 24A). Only the formation of the morpholine ring system with Thr1 properly positions the P1 side chain of ONX 0914 in the S1 specificity site and triggers the reorientation of Met45 as well as further structural changes (Figure 24B). With respect to the distinct affinities of ONX 0914 for the subunits $\beta 5 \mathrm{c}$ and $\beta 5 \mathrm{i}$, the following model for ONX 0914 binding to $\mathrm{cCP}$ and $\mathrm{iCP} \beta 5$ subunits is proposed: In both active sites $\beta 5 \mathrm{i}$ and $\beta 5 \mathrm{c}$ hemiketal formation of ONX 0914 with $\mathrm{Thr}^{\gamma} \mathrm{O}^{\gamma}$ is possible without any sterical hindrance. However, whereas the P1 side chain of ONX 0914 can immediately adopt its final position in the $\mathrm{S} 1$ pocket of subunit $\beta 5 \mathrm{i}$ and thereby form the morpholine ring system as well as the antiparallel $\beta$ sheet, this is not possible in subunit $\beta 5 \mathrm{c}$. Met45 of subunit $\beta 5$ c sterically hampers full binding of the P1 residue to the S1 pocket and has to be dislocated to enable covalent modification of Thr1. Consequently, the energy barrier for the morpholine ring formation in subunit $\beta 5 \mathrm{c}$ is higher and the back-reaction leading to a restored and catalytically active Thr1 in subunit $\beta 5 \mathrm{c}$ is more likely than in $\beta 5 \mathrm{i}$. Thus, the increased probability of ONX 0914 to modify the Thr 1 of subunit $\beta 5 \mathrm{i}$ compared to $\beta 5 \mathrm{c}$ provides the explanation for its lower $\mathrm{IC}_{50}$ value and its selectivity for subunit $\beta 5 \mathrm{i}$.

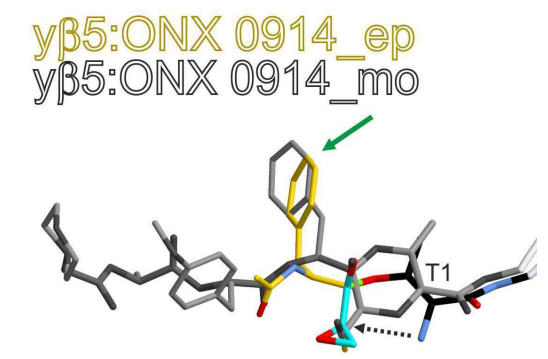

Figure 25 Docking of ONX 0914 to the active site Thr1. Structural superposition of ONX 0914 in its epoxide (ep; yellow) and morpholine (mo; grey) state bound to Thr1 of subunit y $\beta 5$. The green arrow indicates that the positions of the $\mathrm{P} 1$ side chain differ in the reaction intermediate and the reaction product; the black dashed arrow marks the position where the free $\mathrm{N}$-terminus of $\mathrm{y} \beta 5$ attacks the epoxide to form a morpholine ring system. Adapted from Huber et al., 2012 ${ }^{[32]}$. 


\subsection{Yeast mutagenesis studies on the subunits $\beta 5 \mathrm{c}, \beta 5 \mathrm{i}$ and $\beta 5 \mathrm{t}$}

The crystal structures of the murine $\mathrm{cCP}$ and $\mathrm{iCP}$ demonstrated that the proteasomal $\beta$ subunits are structurally conserved from archaea to mammals. Based on the overall identical folds and similar main chain tracings of $\beta 5$ subunits (Figure 26), the yCP can be used as a model system for analysing the functional impact of single amino acid exchanges between the $\mathrm{cCP}, \mathrm{iCP}$ as well as tCP by mutagenesis.
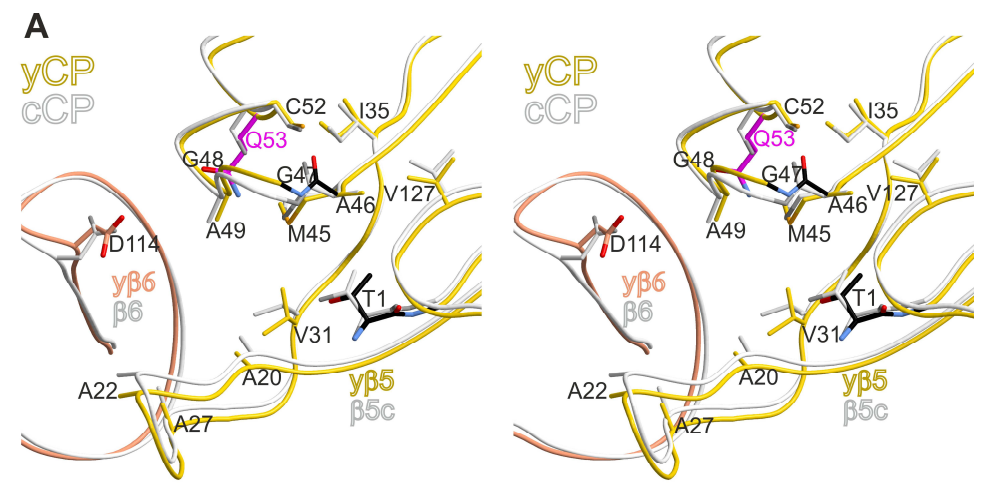

B
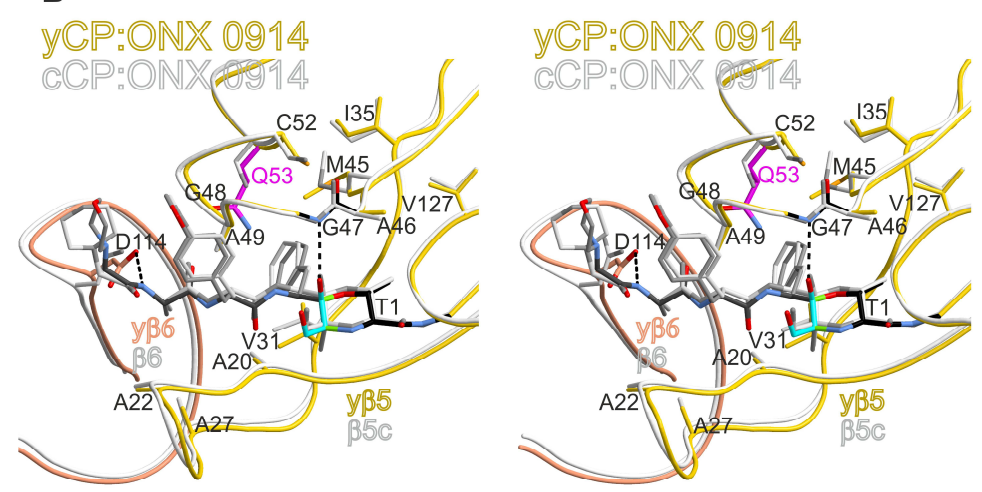

C
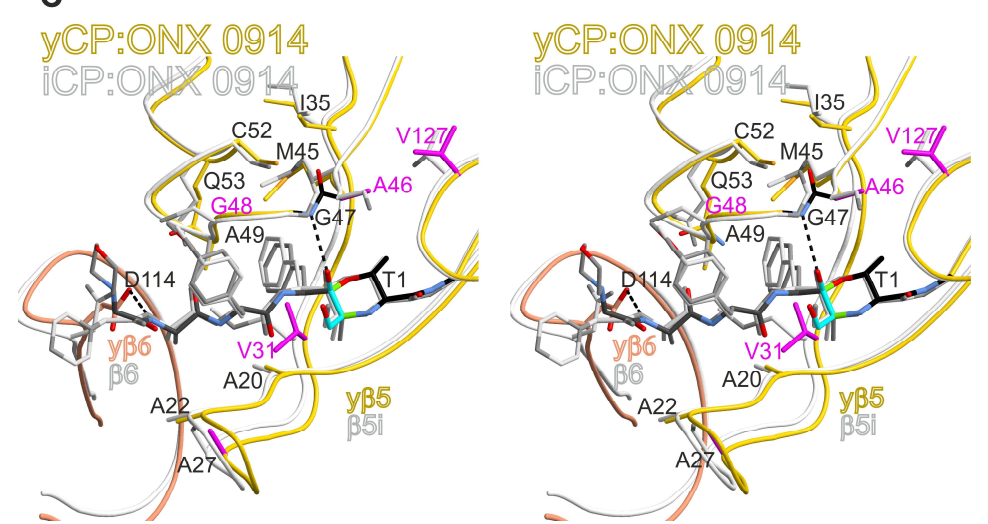

Figure 26 Structural comparison of the yeast subunit $\mathrm{y} \beta 5$ and the murine active sites $\beta 5 \mathrm{c}$ and $\beta 5 \mathrm{i}$ of $\mathrm{cCP}$ and iCP, respectively (stereo view). (A) Superposition of the subunits y $\beta 5$ and $\beta 5 \mathrm{c}$ in their ligand-free states and (B) their ligand-bound states depict the structural similarity of both. Panel (C) additionally illustrates the comparison of the ONX 0914 bound active sites y $\beta 5$ and $\beta 5$ i. Amino acids are numbered for the yCP. Residues that are characteristic of the $\mathrm{yCP}$ subunit y $\beta 5$ are coloured in magenta. Gly47 and Thr1 are shown in black. The ligand is highlighted in grey. Hydrogen bonds are indicated by black dashed lines. Adapted from Huber et al., 2012 $2^{[32]}$. 


\subsubsection{Mimicking the $\beta 5 \mathrm{c}$ active site}

The cCP and iCP crystal structures depicted that the orientation of Met45 is crucial for the size of the S1 specificity pocket in $\beta 5$ subunits (Figure 16; Figure 21). While favourable van der Waals interactions of Met45 with Gln53 contribute to the formation of a large S1 site in subunit $\beta 5 \mathrm{i}$, Ser53 cannot stabilize Met45 in subunit $\beta 5 \mathrm{c}$ (Figure 16) and causes a diminished $\mathrm{S} 1$ pocket in subunit $\beta 5 \mathrm{c}$. This model is supported by the strong conservation of Ser53 in $\beta 5 \mathrm{c}$ subunits, Gln53 in $\beta 5$ i entities and Ala53 in $\beta 5$ t active sites ${ }^{[32]}$. Notably, the yeast y $\beta 5$ subunit incorporates Lys32, a hallmark of $\beta 5$ c entities, as well as the $\beta 5 i$ characteristic residue Gln53, leading to a similar S1 pocket architecture like in $\beta 5 \mathrm{c}$. Hence, subunit y $\beta 5$ appears to represent a chimera of the $\beta 5 \mathrm{c}$ and $\beta 5 \mathrm{i}$ active sites at least with respect to the amino acids 32 and 53. Indeed, the $\mathrm{IC}_{50}$ value of $\mathrm{ONX} 0914$ for subunit $\mathrm{y} \beta 5$ was determined to $\sim 0.5 \mu \mathrm{M}$, while it was $\sim 1 \mu \mathrm{M}$ for $\beta 5 \mathrm{c}$ and $\sim 0.07 \mu \mathrm{M}$ for $\beta 5 \mathrm{i}$ (Figure 17).

The importance of Gln53 for the S1 pocket architecture in the subunits y $\beta 5$ and $\beta 5 \mathrm{i}$ was proven by mutation of Gln53 to Ser in the y 35 active site. The $\mathrm{IC}_{50}$ values of ONX 0914 and bortezomib for the created mutant $\mathrm{CP}$ were $1.32 \mu \mathrm{M}$ and $0.15 \mu \mathrm{M}$, respectively (Figure 27). Remarkably, compared to the wt yCP, ONX 0914 was three-times less potent towards the y $\beta 5$ Q53S mutant, while bortezomib displayed an increased affinity (Figure 27). These findings elucidate that Ser53 enhances binding of ligands with smaller P1 side chains such as Leu (bortezomib) over inhibitors with bulky residues like Phe (ONX 0914). Wt y/55 shows only slight preference for bortezomib, but the mutation Q53S significantly increases the selectivity for this compound (Figure 27). In agreement, subunit $\beta 5 \mathrm{c}$ was reported to be far more susceptible to inhibition by bortezomib $\left(\mathrm{IC}_{50}: 7 \mathrm{nM}^{[74]}\right.$ ) than ONX $0914\left(\mathrm{IC}_{50}: 236-1000 \mathrm{nM}\right.$ depending on the method ${ }^{[32,37]}$ ). For structural analysis of the Q53S mutant X-ray

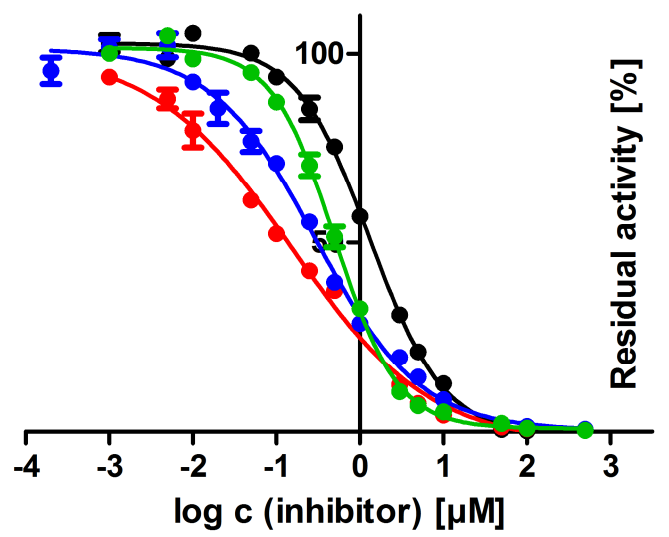

\begin{tabular}{|c|c|}
\hline B5-subunit & $I C_{50}[\mu M]$ \\
\hline y $\beta 5$ bortezomib & $0.297 \pm 0.058$ \\
\hline yß5 ONX 0914 & $0.499 \pm 0.038$ \\
\hline y $\beta 5$ Q53S bortezomib & $0.152 \pm 0.048$ \\
\hline yß5 Q53S ONX 0914 & $1.319 \pm 0.117$ \\
\hline \multicolumn{2}{|l|}{$\multimap$ y $\beta 5$ Bortezomib } \\
\hline \multicolumn{2}{|l|}{$\multimap$ yß5 ONX 0914} \\
\hline \multicolumn{2}{|c|}{$\multimap$ y $\beta 5$ Q53S Bortezomib } \\
\hline \multicolumn{2}{|c|}{$\rightarrow$ y 35 Q53S ONX 0914} \\
\hline
\end{tabular}

Figure 27 Inhibition of wt and mutant yCPs by ONX 0914 and bortezomib. Residual proteolytic activities were measured in triplicate upon exposure to different inhibitor concentrations with the fluorogenic substrate Suc-Leu-Leu-Val-Tyr-AMC and normalized to a DMSO treated control. $\mathrm{IC}_{50}$ values were deduced from fitted data. 
data were collected in the presence and the absence of both ligands (Table 16). The mutant y $\beta 5$ subunit Q53S displays high structural identity to its wt counterpart (r.m.s.d. $\mathrm{C}_{\alpha}$ y $\beta 5 / y \beta 5$ Q53S: $0.2 \AA$; Figure 28A) and binding of bortezomib and ONX 0914 induces structural changes similar to y $\beta 5$ and $\beta 5$ c (r.m.s.d. $C_{\alpha}$ y $\beta 5 Q 53 S / y \beta 5 Q 53 S: O N X$ 0914: $0.76 \AA$; r.m.s.d. $\mathrm{C}_{\alpha}$ yß5Q53S/yß5Q53S: bortezomib: $0.62 \AA$ A Figure 28B, C, F). However, ONX 0914 leads to a more severe displacement of the sulphur atom of Met45 than bortezomib (Figure 28D), hereby explaining the reduced affinity of ONX 0914 for subunit $y \beta 5 Q 53 S$ compared to bortezomib (Figure 27). Removal of Gln53 leads to an insufficient stabilization of Met45 in the ligand-bound state and hence, more energy is required for bulky P1 side chains to reorient Met45 and to keep distance to it. The propensity of Met45 to adopt its normal conformation is likely to hinder particularly spacious ligands such as ONX 0914 from binding irreversibly to Thr1. In conclusion, although the substitution Q53S causes no obvious structural differences and amino acid 53 is not directly involved in ligand binding, its side chain length yet significantly affects the affinity of ligands.

A

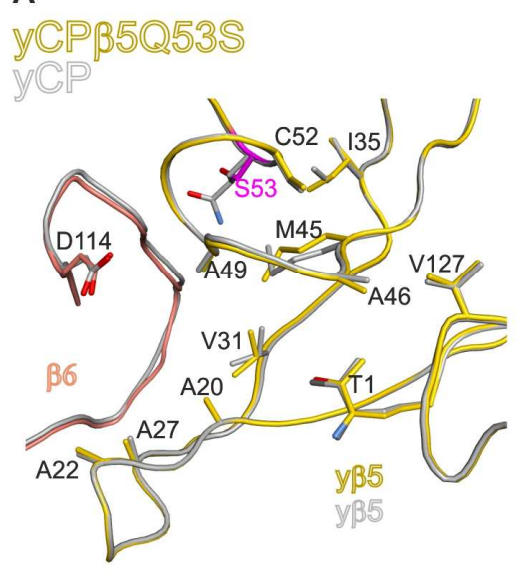

D

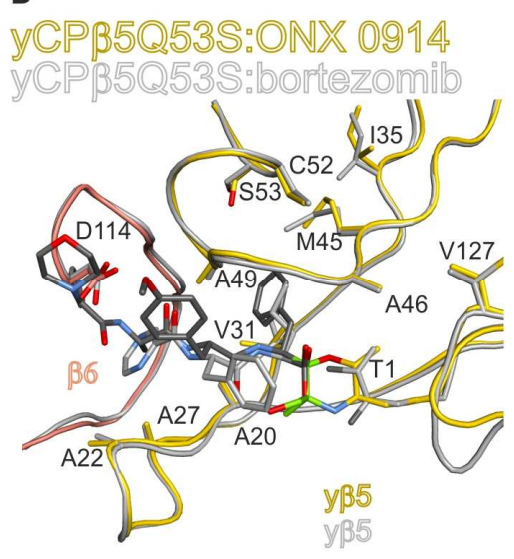

B

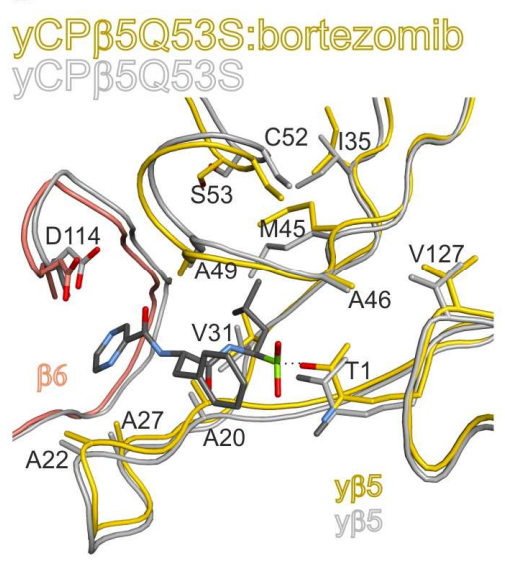

E
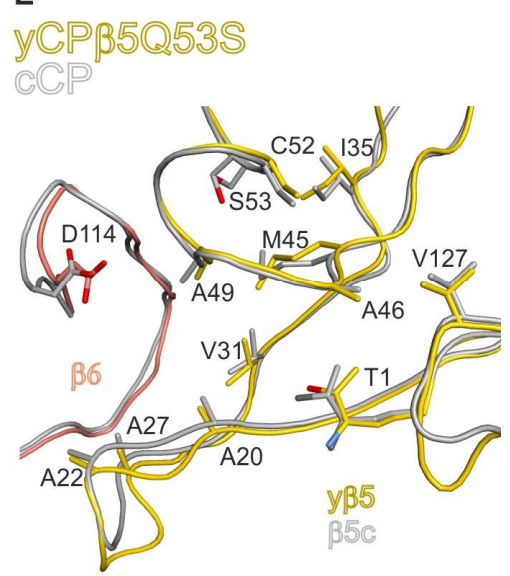

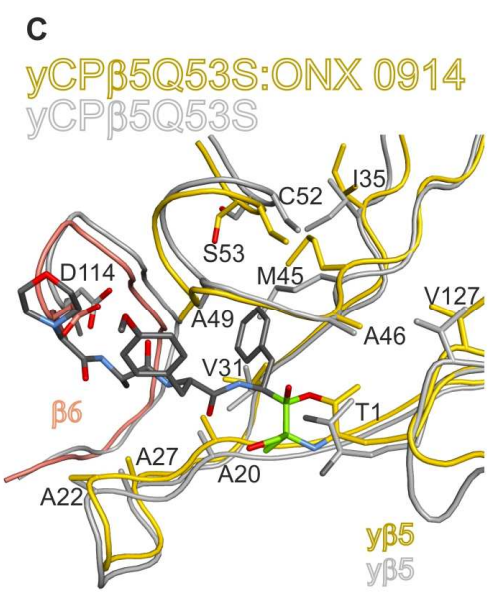

$\mathbf{F}$
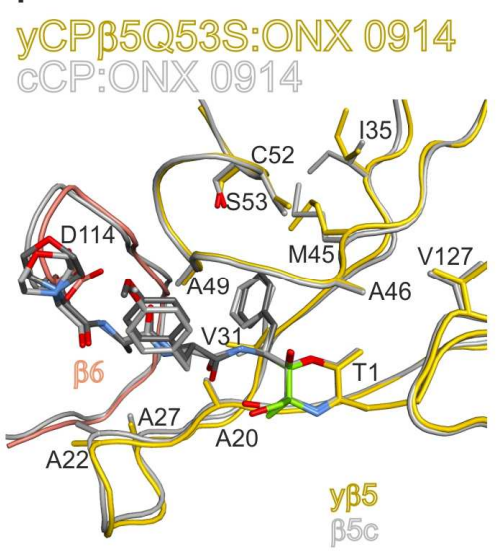

Figure 28 Structural analysis of the yCP $\beta 5$ mutant Q53S. (A-D) Superposition of (un-)liganded wt and mutant yeast $\beta 5$ active sites; (E, F) Comparison of the yCP $\beta 5$ mutant Q53S with the murine cCP. Amino acid numbers are given for the $\mathrm{yCP} \beta 5$ mutant $\mathrm{Q} 53 \mathrm{~S}$; sequence differences between superimposed structures are highlighted in magenta; the electrophilic head groups of inhibitory compounds (grey) are coloured in green. 


\subsubsection{Analysis of the $\beta 5 \mathrm{i}$ substrate binding channel}

The comparison of the crystal structures of $\mathrm{cCP}$ and $\mathrm{iCP}$ suggests that van der Waals interactions of Met45 with the aliphatic side chain of Gln53 in subunit $\beta 5$ i promote the formation of a spacious S1 pocket (Figure 16). Although subunit y $\beta 5$ harbours a Gln53, Met45 adopts a conformation similar to subunit $\beta 5 \mathrm{c}$. This observation provoked the question of which additional amino acids in proximity to the active site contribute to the distinct conformations of Met45.

To identify key residues that lead to the differently sized S1 pocket in the $\beta 5 \mathrm{i}$ substrate binding channel, numerous point mutations were introduced in subunit y $\beta 5$. The following amino acid exchanges aimed at mimicking the murine (or human) $\beta 5 \mathrm{i}$ subunit: A27S, (V31M,) K32N, A46S, G48C, T57R, K71G and V127T (Figure 8; Table 3).

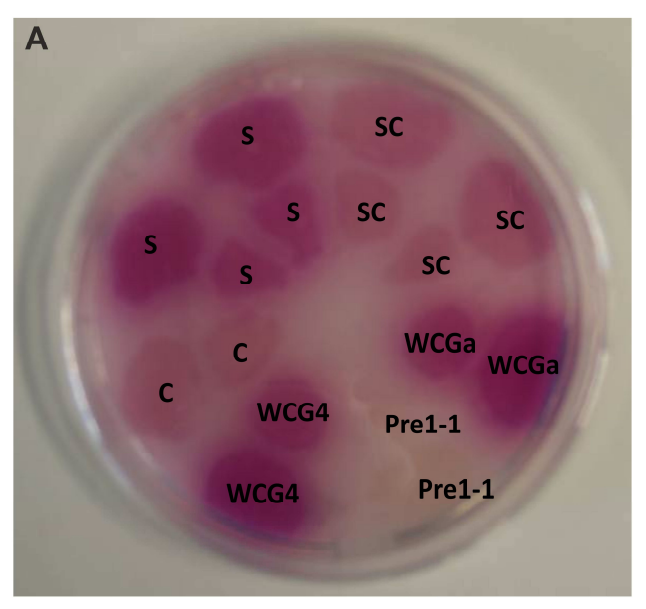

C

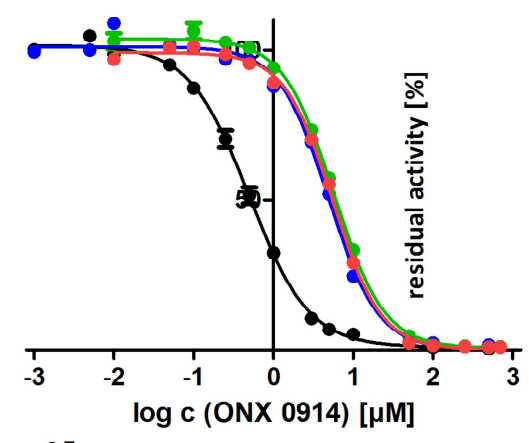

- $\mathrm{y} \beta 5$

$\rightarrow$ yß5 A27S K32N A46S G48C T57R K71G V127T

$\rightarrow$ yß5 A27S K32N M45A A46S G48C T57R K71G V127T

$\rightarrow$ y 35 A27S K32N M45V A46S G48C T57R K71G V127T

B

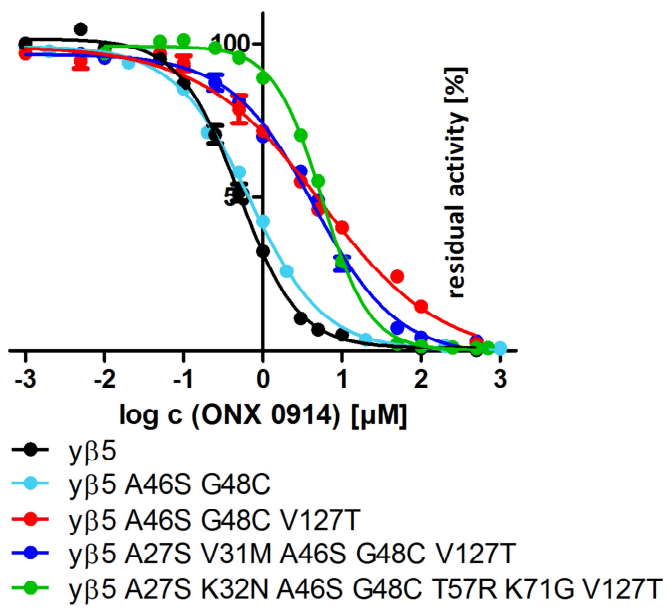

D

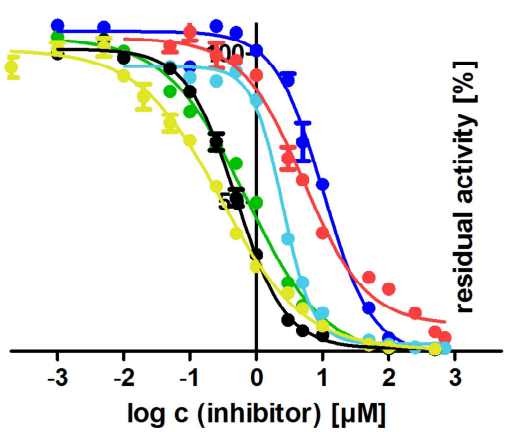

y $\beta 5$ ONX 0914

yß5 M45R ONX 0914

y 55 I35T M45R ONX $0914 \rightarrow y \beta 5$ I35T M45R bortezomib

Figure 29 Activity and inhibition assays of mutant and wt yCPs. (A) Overlay test for the ChTL activity of yCPs using the chromogenic substrate Cbz-Gly-Gly-Leu-pNA. Tested mutants are termed according to Table 3. The yeast strains WCG4a and WCG4a pre1-1 served as positive and negative controls, respectively. (B-D) The indicated mutant CPs that aimed at imitating the iCP subunit $\beta 5 \mathrm{i}$ were tested in triplicate for their inhibition by

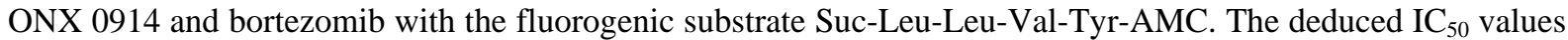
are given in Table 14. 
Remarkably, most mutant yeast strains suffer from a significant growth phenotype, which is probably caused by an impaired $\beta 5$ activity, as observed in a proteolysis assay with the chromogenic substrate Cbz-Gly-Gly-Leu-pNA (Figure 29A). Hereby, the single point mutation G48C sufficed to markedly attenuate the ChTL activity. Cys48 might restrict the flexibility of the loop segment 46-49 and thereby hamper substrate and inhibitor binding. While the exchange A46S alone did not affect CP activity and its combination with G48C had only minor effects on the $\mathrm{IC}_{50}$ value of ONX 0914, additional introduction of Thr127 strongly impaired ligand binding (Table 14). The hydrogen bond between Ser46 and Thr127 that has also been observed for the iCP structure additionally enhances the rigidity of the loop region 46-49 and thereby might constrict ligand binding. Intriguingly, mutagenesis of additional residues (A27S, K32N, V31M, T57R, K71G) had neither further influence on the affinity of ONX 0914 for the y 35 active site (Figure 29B) and nor did it lead to the reorientation of Met45 as observed in the unliganded subunit $\beta 5$ i (Figure 30E; Table 17).

\begin{tabular}{|c|c|c|}
\hline B5-subunit & Inhibitor & $I C_{50}[\mu M]$ \\
\hline y $\beta 5$ & bortezomib & $0.297 \pm 0.058$ \\
\hline y $\beta 5$ & ONX 0914 & $0.499 \pm 0.038$ \\
\hline y $\beta 5$ SC & ONX 0914 & $0.673 \pm 0.055$ \\
\hline y $\beta 5$ SCT & ONX 0914 & $5.379 \pm 1.839$ \\
\hline yß5 SMSCT & ONX 0914 & $4.416 \pm 0.771$ \\
\hline y 35 SNSCRGT & bortezomib & $1.807 \pm 0.168$ \\
\hline yß5 SNSCRGT & ONX 0914 & $5.580 \pm 0.307$ \\
\hline y $\beta 5$ SNASCRGT & bortezomib & $3.078 \pm 0.402$ \\
\hline y $\beta 5$ SNASCRGT & ONX 0914 & $5.706 \pm 0.321$ \\
\hline y $\beta 5$ SNVSCRGT & bortezomib & $2.606 \pm 0.266$ \\
\hline y $\beta 5$ SNVSCRGT & ONX 0914 & $4.911 \pm 0.409$ \\
\hline y $\beta 5 \mathrm{R}$ & bortezomib & $2.508 \pm 0.178$ \\
\hline y $\beta 5 R$ & ONX 0914 & $5.185 \pm 1.097$ \\
\hline y $\beta 5$ TR & bortezomib & $0.706 \pm 0.115$ \\
\hline y $\beta 5 \mathrm{TR}$ & ONX 0914 & $10.37 \pm 2.134$ \\
\hline
\end{tabular}

Table $14 \mathrm{IC}_{50}$ values for yCP mutants that partially mimic either the primary sequence or the structural features of the iCP subunit $\mathbf{\beta 5 i}$. Mutants are termed according to Table 3.

Hence, the key features of subunit $\beta 5$ i could so far not be reconstituted in yeast, even though all amino acids in the active site surrounding were identical to the murine/human subunit $\beta 5 \mathrm{i}$ (Figure 30E). Consequently, rather the structural differences than the primary sequences of the $\beta 5 \mathrm{i}$ and $\beta 5 \mathrm{c} / \mathrm{y} \beta 5$ substrate binding channels are responsible for the selectivity of ONX 0914. In order to mimic the enlarged S1 pocket of subunit $\beta 5 \mathrm{i}$, Met45 was substituted by either alanine or valine, but intriguingly these mutant CPs exhibited only poor affinity for both ONX 0914 and bortezomib (Table 14). Probably in the absence of a 
A

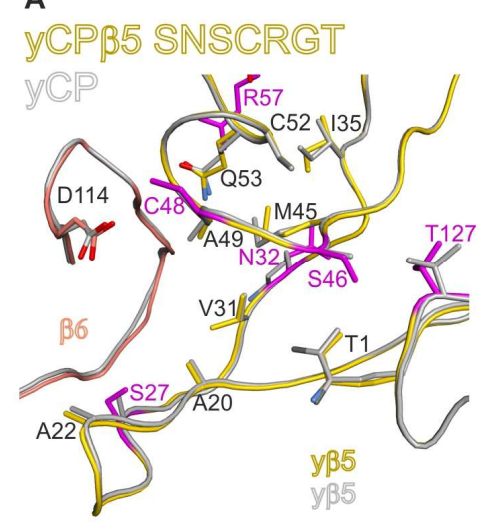

D
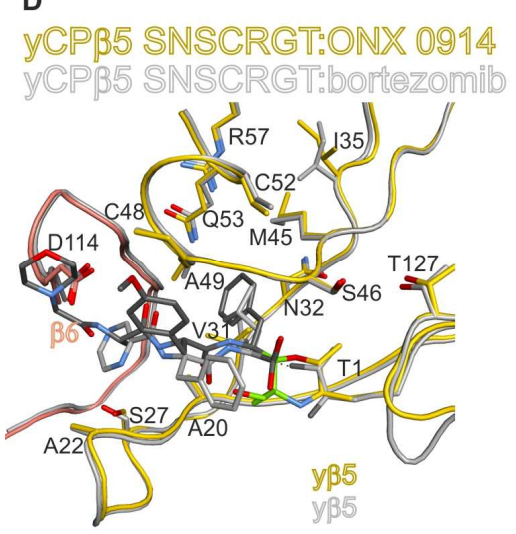

B

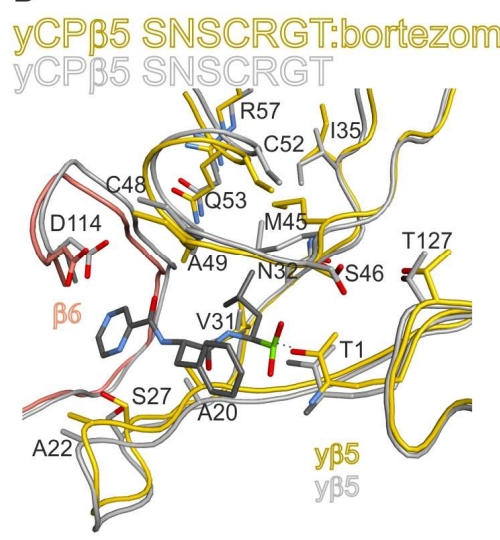

E

YCPBS SNSCRGT

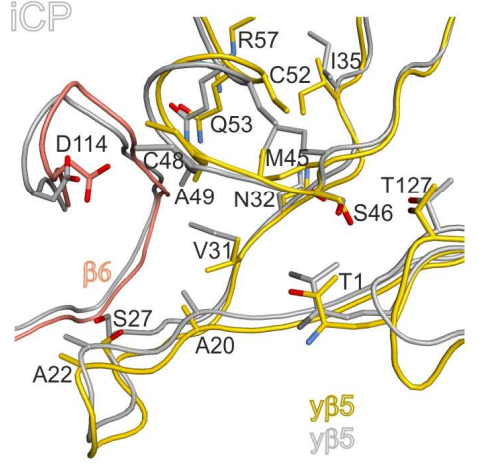

C

YCPBS SNSCRGT:ONX 0914

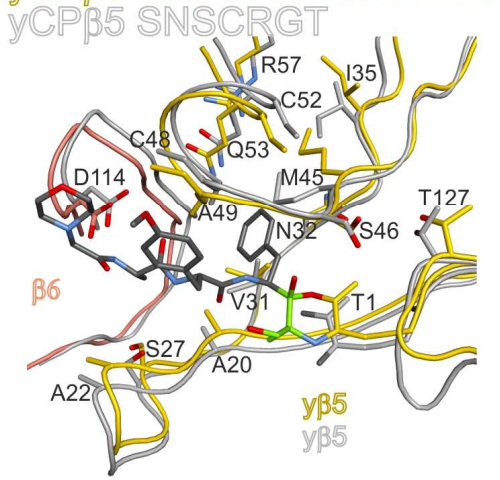

$\mathbf{F}$
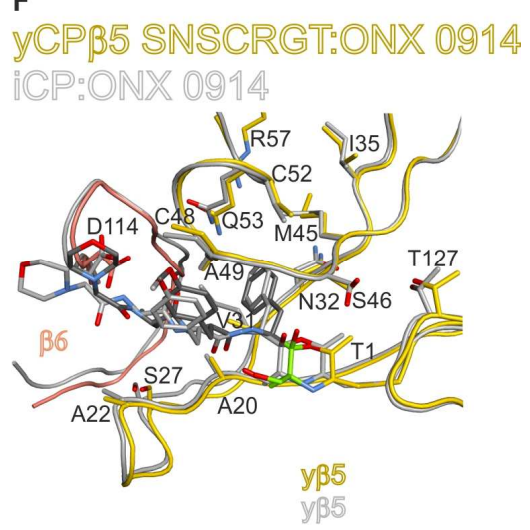

Figure 30 Crystal structure analysis of $\mathbf{\beta 5 i}$ mimicking yCPs. Mutant y $\beta 5$ substrate binding channels are superimposed onto wt $\beta 5$ active sites of wt $\mathrm{yCP}$ and iCP to depict structural similarities and differences. Amino acid labellings are indicated for mutant CPs; amino acid mutations are highlighted in magenta and the electrophilic headgroups of inhibitors are shown in green.

prolonged linear aliphatic side chain such as Met45 ligands cannot be sufficiently stabilized in the S1 pocket. These results are also in line with reports on the bortezomib resistance conferring character of Val45 ${ }^{[72 \mathrm{c}]}$ and are indicative of the highly sophisticated architecture of the proteasome.

Concomitantly, structural characterization of the $y \beta 5$ mutants M45R and I35T M45R that were previously analysed ${ }^{[84]}$ and that are unrelated to the $\mathrm{iCP}$ revealed an interesting feature. The amino acid exchange M45R reduces the ChTL activity by electrostatic repulsion of apolar amino acids by Arg45, but by the additional mutation I35T the ChTL activity is regained. Ligand complex structures of the mutant M45R with bortezomib and ONX 0914 visualized that only binding of the $\alpha^{\prime}, \beta$ 'epoxyketone causes major structural changes of Arg45 (r.m.s.d. $\mathrm{C}_{\alpha}$ yß5M45R/yß5M45R:ONX 0914: $0.82 \AA$ A; r.m.s.d. $\mathrm{C}_{\alpha}$ yß5M45R/ yß5M45R:bortezomib: $0.43 \AA$; Figure 31B, C; Table 18). Hence, the poor affinity of ONX 0914 is presumably caused by sterical hindrance and the opposing polarity in the S1 pocket, while binding of bortezomib appears to be solely hampered by electrostatic repulsion. 
A

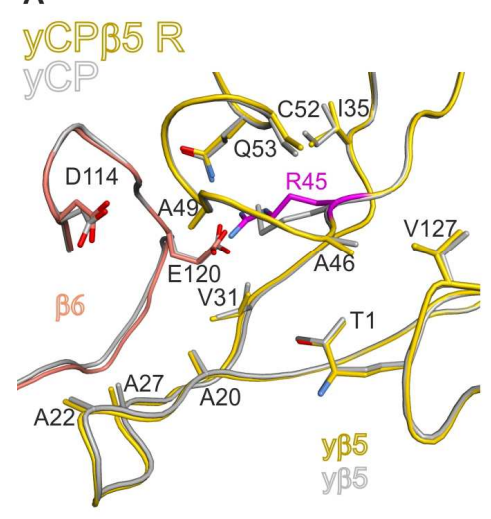

D

\section{yCPBS R:ONX 0.94}

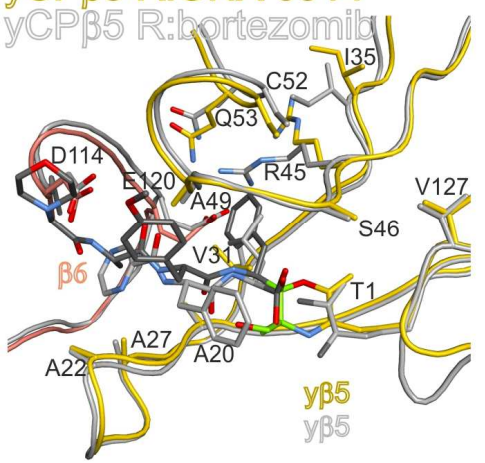

G

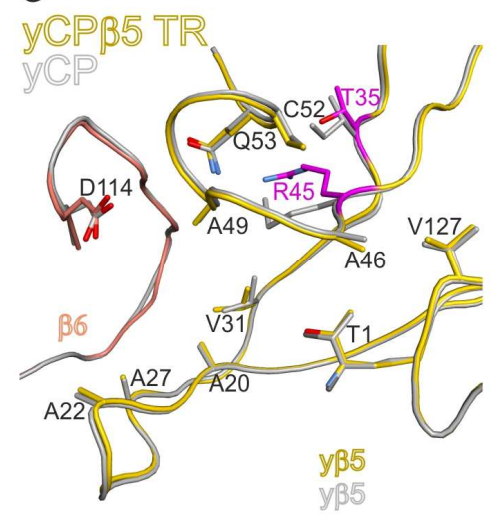

J

yCPB5 TR:ONX (0914

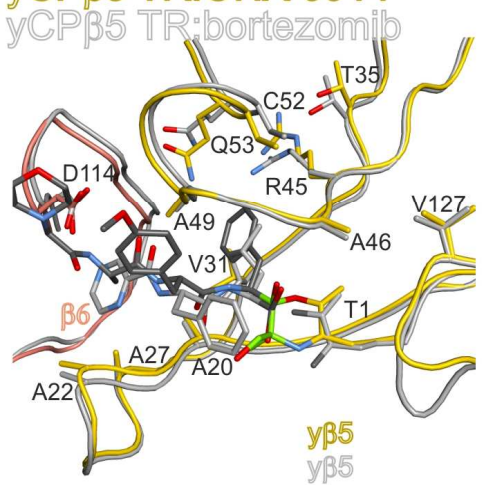

B

yCPB5 R:bortezonilb

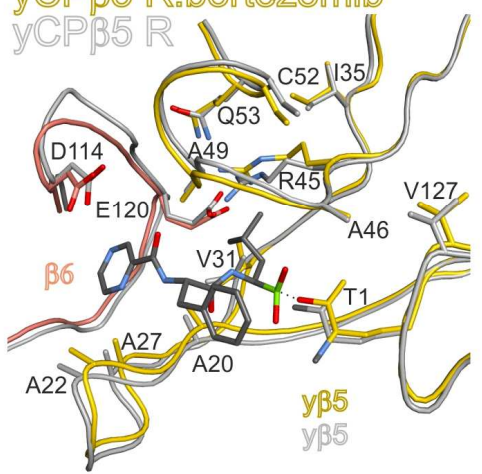

E

yCPB5 R

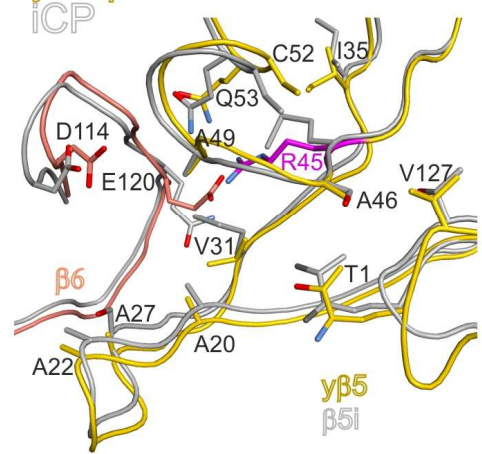

H

\section{yCPB5 『R:bortezoñib}

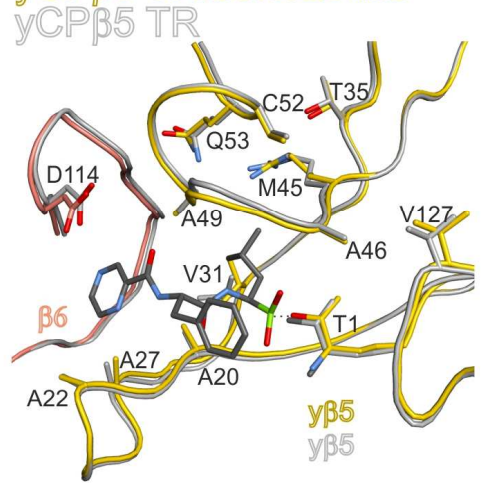

K

yCPB5 『R

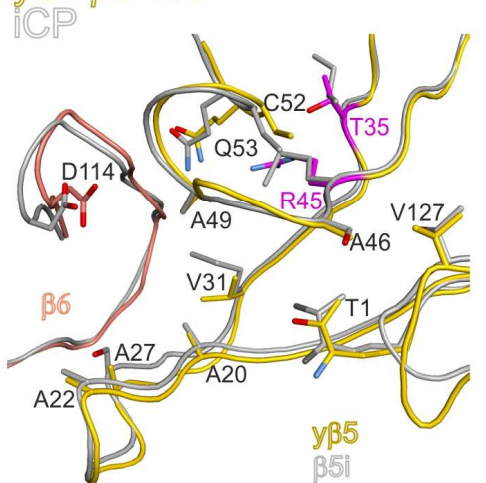

C

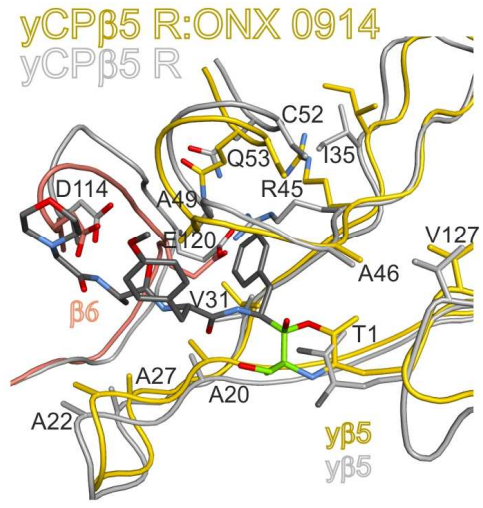

F

yCPB5 R:ONX (0914

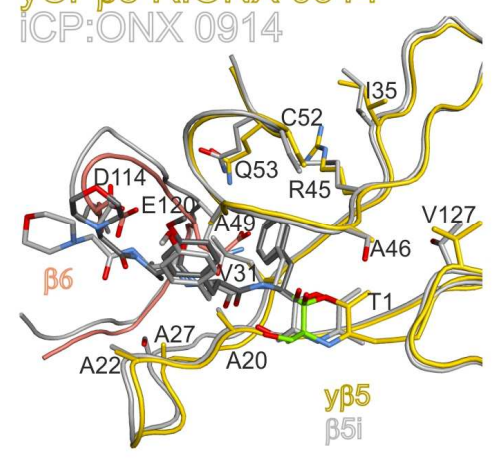

I

yCPB5 TR:ONX (094 4

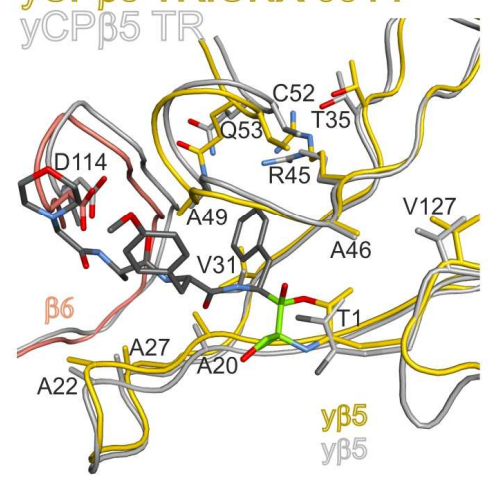

L

YCPBS 『R: ONX (09)14

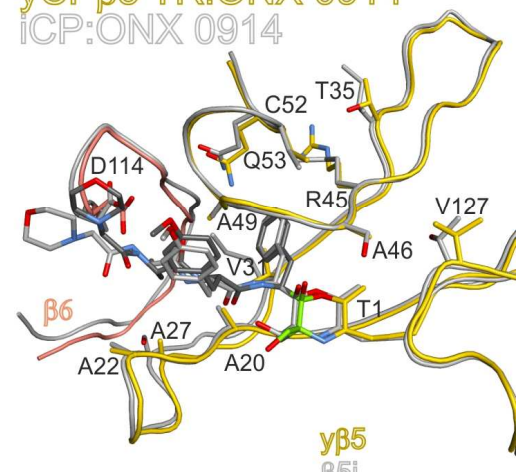

Figure 31 Crystal structures of mutant CPs. Mutant y $\beta 5$ substrate binding channels are superimposed onto $\beta 5$ active sites of wt yCP and iCP to depict structural similarities and differences in the presence and absence of inhibitor. Amino acid residues of mutant CPs are labelled; amino acid mutations are highlighted in magenta and the electrophilic headgroups of inhibitors are shown in green. 
Interestingly, the restored ChTL activity of the double mutant relies on a strong hydrogen bond $\left(2.8 \AA\right.$ ) between $\operatorname{Thr} 35 \mathrm{O}^{\gamma}$ and $\operatorname{Arg} 45 \mathrm{~N}^{\varepsilon}$ that rearranges Arg45 and enlarges the size of the $\mathrm{S} 1$ pocket similar to $\beta 5 \mathrm{i}$ (Figure $31 \mathrm{G}, \mathrm{K}$ ). Due to the spacious S1 site the double mutant displayed a 3.5 times enhanced affinity for bortezomib compared to the $y \beta 5 \mathrm{M} 45 \mathrm{R}$ mutant (Table 14) and the ligand does not induce any structural rearrangement (r.m.s.d. $\mathrm{C}_{\alpha}$ yß5TR/yß5TR:bortezomib: $0.35 \AA$; Figure $31 \mathrm{H}$; Table 19). This finding proves that indeed the size of the S1 pocket affects the inhibitory potency of compounds. Still, the mutant yCP I35T M45R is dramatically less susceptible to ONX 0914 than the wt yCP (Table 14), because accommodation of its bulky phenyl side chain is electrostatically hindered by the guanidine group of Arg45 (Figure 31I). As confirmed by crystallographic analysis ligand binding pushes Arg45 $1 \AA$ further away from the phenylgroup of ONX 0914, thereby significantly enlarging its distance to $\operatorname{Arg} 45$ up to $5 \AA$ (r.m.s.d. $\mathrm{C}_{\alpha}$ yß5TR/yß5TR:ONX 0914: 0.69 $\AA$; Figure 31I). A similar distance of 4.8-5.2 $\AA$ is observed for Arg45 and the P1 leucine of bortezomib bound to the yCP I35T M45R mutant (Figure 31H). In conclusion, the strongly reduced affinity of ONX 0914 for the double mutant discloses the enormous effects of electrostatic repulsion on ligand binding (see also section 6.4.3) and highlights the perfect suitability of the Met side chain for establishing a ChTL activity.

\subsubsection{Probing subunit $\beta 5 t$}

The thymoproteasome incorporates the tCP-specific subunit $\beta 5 \mathrm{t}$ that - unlike the $\beta 5 \mathrm{c}$ and $\beta 5 \mathrm{i}$ active sites - harbours a more hydrophilic substrate binding channel ${ }^{[21 c]}$. In particular the amino acid residues Ser20, Ser31, Thr45, Thr48 and Ser49 (Figure 8) enhance the polarity of the unprimed S1 and S2 pockets and favour cleavage C-terminally of charged residues. In contrast, the S3 pocket is lined with the hydrophobic residues Cys22 and Ala27. Similarly to subunit $\beta 5$ i, Ser46 might increase the polarity around the active site Thr1 and the oxyanion hole (see also section 6.2.3). Val127, however, is not suited to form a hydrogen bond network with Ser46 like it is observed for subunit $\beta 5$ i.

Due to limited biological samples no structural information on the tCP is available so far. Therefore, this study aimed at mimicking the $\beta 5 \mathrm{t}$ active site in yeast by mutagenesis. A stepwise approach was used to introduce the mutations A20S, A22C, V31S, M45T, A46S and G48T into subunit y $\beta 5$ (Table 3). Most of the created mutants were characterized by a reduced growth rate and displayed a dramatically decreased ChTL activity of the y $\beta 5$ subunit 
A

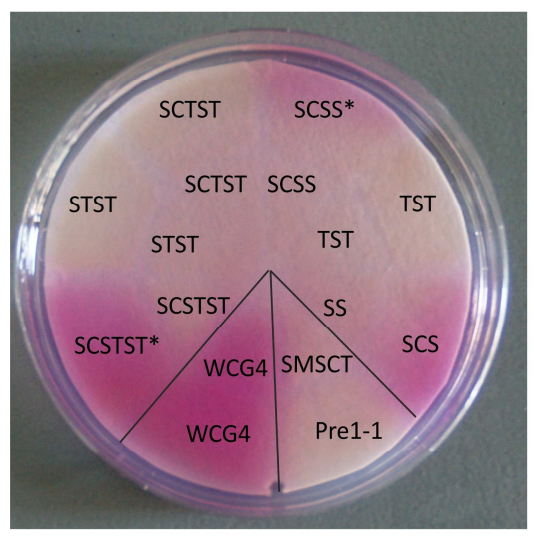

C

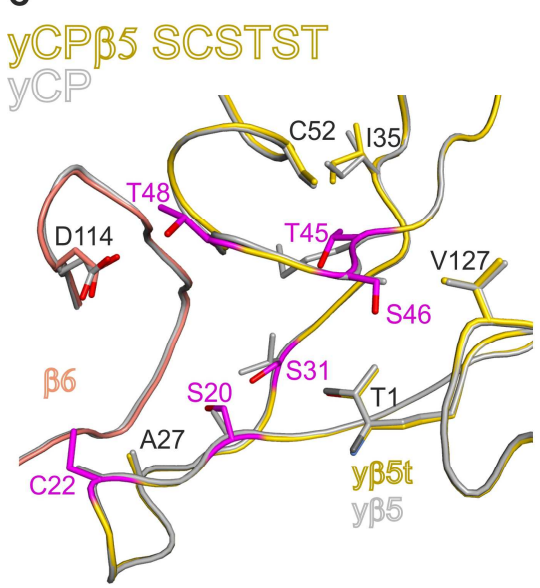

B

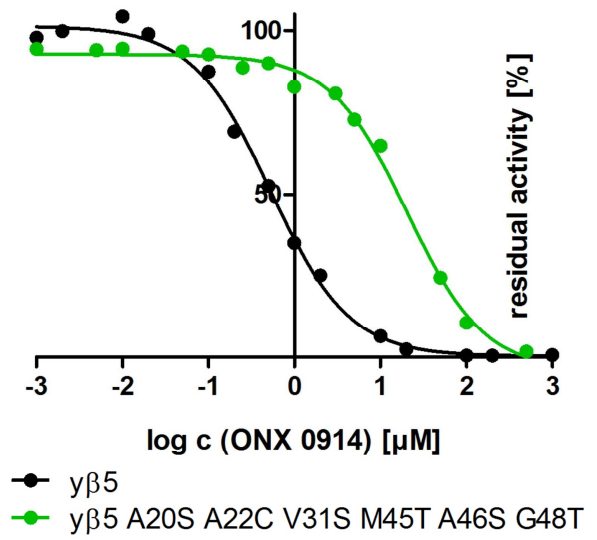

D

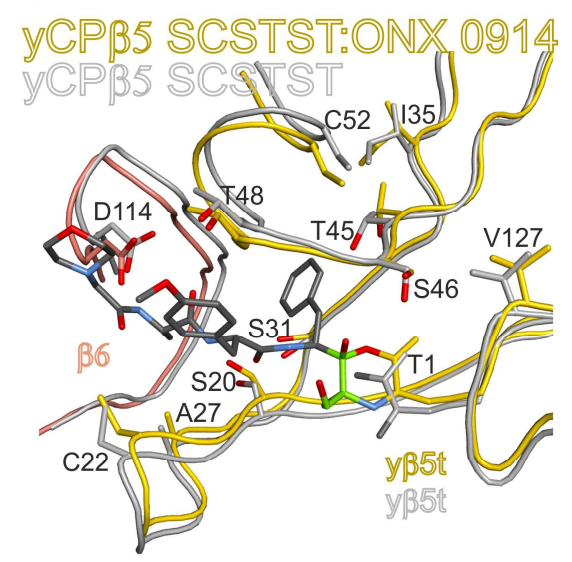

Figure 32 Structural examination of a $\beta 5 \mathrm{t}$ mimicking yCP. (A) An overlay assay using the chromogenic substrate Cbz-Gly-Gly-Leu-pNA shows the marked reduction in ChTL activity upon introduction of $\beta 5$ t-specific mutations. Wildtype yeast (WCG4a) served as a positive control, whereas the $\beta 5$ i-mimicking y $\beta 5$ mutant SMSCT and the CP mutant Pre1-1, which is lacking ChTL activity, were used as negative controls. Mutant clones marked with a star showed unspecific staining. For abbreviations of mutants see Table 3. (B) Suc-LeuLeu-Val-Tyr-AMC was used as a fluorogenic substrate to assess the inhibition of the mutated subunit $\beta 5$ by the epoxyketone ONX 0914. The affinity of ONX 0914 is strongly attenuated in the mutant CP (C, D) Comparison of the wildtype and mutant (SCSTST) y $\beta 5$ substrate binding channels depicts the differences in their primary sequences (magenta) and illustrates structural changes upon ligand binding. Amino acids are labelled for the mutant CP.

(Figure 32A). Moreover, the $\mathrm{IC}_{50}$ value of ONX 0914 for the SCSTST mutant y $\beta 5$ active site strongly increased to $20.57 \mu \mathrm{M} \pm 3.78 \mu \mathrm{M}$ compared to $0.499 \mu \mathrm{M}$ for wt $\mathrm{y} \beta 5$. Even though the S1 pocket of the y 35 SCSTST mutant is enlarged, the opposing forces between the hydrophobic side chains of ONX 0914 and the charged amino acid lining of the substrate binding pockets of the y $\beta 5$ mutant SCSTST strongly impair binding of the ligand.

Despite the reduced affinity of ONX 0914 for this mutant yCP structural data could be obtained in the absence and the presence of the compound (Table 20). Positive $F_{O}-F_{C}$ density maps proved the incorporation of all mutations. The wt and mutant y $\beta 5$ active sites superimpose well and show no structural changes for the mutant subunit (r.m.s.d. $\mathrm{C}_{\alpha}$ y $\beta 5 / y \beta 5$ t: $0.2 \AA$ ). Comparison of the ligand-free and ligand-bound states of the $\beta 5$ t-like 
substrate binding channel depicts the turn away of Thr45 from the hydrophobic ligand ONX 0914. In addition, strong backbone distortions (r.m.s.d. $C_{\alpha}$ y $\beta 5 t / y \beta 5 t: O N X ~ 0914$ : $0.72 \AA$ ) are observed upon ligand binding even though no obvious clashes with the protein occur. The reason for this tremendous backbone shift upon inhibitor binding cannot be deduced from the mutant structures and has to be further investigated by structural analysis of the tCP. 


\section{Discussion}

The here presented crystallographic analysis of the murine cCP and $\mathrm{iCP}$ emphasizes the biological impact of subtle differences that are not predictable from sequence alignments and that can only be resolved by structural data. However, owing to the applied methodology, the results presented provide no insights into the dynamics of the $20 \mathrm{~S}$ proteasome that might have substantial effects on substrate binding as well as enzyme inhibition.

\subsection{Structural and functional differences between the three types of CPs}

The high structural identity of the unprimed $\beta 2 \mathrm{c}$ and $\beta 2 \mathrm{i}$ substrate binding channels implicates similar cleavage preferences, leading to the generation of MHC I ligands with neutral or basic C-terminal amino acids ${ }^{[111]}$. Hence, the $\mathrm{CCP}$ and $\mathrm{iCP}$ structures elicit the question of why $\beta 2 \mathrm{i}$, which is the only $\mathrm{i}$ subunit that is not encoded on the MHC gene cluster, is sequestered into iCPs. For the elucidation of the distinct physiological roles of the $\beta 2 \mathrm{c}$ and $\beta 2 \mathrm{i}$ subunits sitespecific probes or subunit-selective compounds would be required. So far, mice lacking subunit $\beta 2 \mathrm{i}$ were demonstrated to be not affected by DSS-induced colitis ${ }^{[53 \mathrm{a}]}$ and drugs targeting both TL active sites were proven to sensitize malignant cells for inhibition of the $\beta 5$ active sites of the proteasome ${ }^{[77]}$. Together, these preliminary results suggest a therapeutically relevant function also for the $\beta 2$ subunits, which both exert rather broad substrate specificities.

According to previous suggestions ${ }^{[13]}$, the unprimed substrate binding sites of subunit $\beta 1 i$ are lined with apolar protein side chains that give rise to a Braap activity ${ }^{[33 b]}$. Thus, incorporation of subunit $\beta 1 \mathrm{i}$ into the iCP stimulates the generation of high-affinity MHC I peptides. In agreement, the cytotoxic $\mathrm{T}$ cell responses of $\beta 1 \mathrm{i}$-deficient mice are altered with respect to their antigen specificity ${ }^{[112]}$.

The exchange of subunit $\beta 5 \mathrm{c}$ by $\beta 5 \mathrm{i}$ is suggested to generally enhance peptide bond cleavage due to the unique hydrophilicity in proximity to the active site $\mathrm{Thr} 1$ of subunit $\beta 5 \mathrm{i}^{[32]}$. In particular, the strictly conserved amino acids Ser46 and Thr127 are suggested to stimulate protein degradation. In agreement the $26 \mathrm{~S}$ immunoproteasome was attributed twice the activity of the constitutive $26 \mathrm{~S}$ proteasome ${ }^{[38]}$ and the rates of antigen processing were shown to influence the immunogenicity of epitopes ${ }^{[113]}$. Despite similar primary sequences for the $\beta 5 \mathrm{c}$ and $\beta 5 \mathrm{i}$ substrate specificity pockets both subunits still differ in their cleavage patterns ${ }^{[114]}$. Distinct conformations of Met45 cause subunit $\beta 5 \mathrm{c}$ to preferentially bind tiny 
non-polar amino acids such as alanine or valine in the $S 1$ pocket, while subunit $\beta 5 i$ favours bulky aromatic P1 residues like phenylalanine, tyrosine ${ }^{[115]}$ and tryptophan. These substrate preferences are in line with the published selective fluorogenic AMC-substrates Ac-Trp-LeuAla-AMC for subunit $\beta 5 c$ and Ac-Ala-Asn-Trp-AMC for subunit $\beta 5 \mathrm{i}^{[110]}$. Leucine and isoleucine are supposed to target subunit $\beta 5 \mathrm{c}$ with a slight preference over $\beta 5 \mathrm{i}$. Both presumably displace Met 45 in subunit $\beta 5 \mathrm{c}$, but might also not be sufficiently stabilized in the spacious S1 pocket of subunit $\beta 5$ i during initial ligand docking. By contrast, Tyr fits well in the $\mathrm{S} 1$ pocket of subunit $\beta 5 \mathrm{i}$ and is exceptionally well-stabilized in the $\beta 5 \mathrm{c}$ counterpart by interactions with Ser53 $(\beta 5 \mathrm{c})$ and Ser129 ( $\beta 6)$. Therefore, it is accepted by both $\beta 5 \mathrm{c}$ and $\beta 5 \mathrm{i}$ as a $\mathrm{P} 1$ residue. In conclusion, subunit $\beta 5 \mathrm{i}$ evolved to efficiently support antigen presentation by producing epitopes with a broad range of hydrophobic C-terminal amino acids for tight binding to MHC I receptors. Its outstanding role for antigen generation is corroborated by a $50 \%$ reduction in MHC I levels in $\beta 5$ i-deficient mice ${ }^{[116]}$ and their enhanced predisposition to infections ${ }^{[117]}$. Notably, the deletion of the subunits $\beta 1 \mathrm{i}$ or $\beta 2 \mathrm{i}$ does not affect MHC I $\operatorname{expression}^{[23,118]}$.

Mutagenesis experiments aimed at mimicking the key features of subunit $\beta 5 \mathrm{i}$ in yeast in order to investigate in more detail the subtle differences between the $\beta 5$ active sites. The created mutants addressed all sequence differences in the substrate binding pockets and around the catalytic Thr1. However, none of the mutant CPs was as susceptible to inhibition by ONX 0914 as subunit $\beta 5 \mathrm{i}$ and in agreement their X-ray structures depicted no change in the side chain conformation of Met45. These findings point out that indeed the structural differences between the $\beta 5 \mathrm{c}$ and $\beta 5 \mathrm{i}$ subunits provoke their different substrate specificities. Furthermore, the structural and functional key features of the $\mathrm{iCP}$ are based rather on longrange effects and amino acid networks than on single point mutations. Remarkably, substitution of the y $\beta 5$ subunit by the mammalian $\beta 5$ i entity is lethal to yeast even when using codon-optimized sequences and even when the y $\beta 5$ propeptide replaces the $\beta 5$ i counterpart (unpublished data of W. Heinemeyer). Analysis of the subunit contacts in the yCP and iCP suggest that solely Arg57 might suppress assembly of subunit $\beta 5 \mathrm{i}$ into the $\mathrm{yCP}$ by clashing with the adjacent Arg82 from subunit y $\beta 6$, but even a $\beta 5$ i R57T mutant could not be successfully incorporated into the yCP (unpublished results of W. Heinemeyer).

Unlike the $\beta 5 \mathrm{i}$ subunit, the $\beta 5 \mathrm{c}$ active site presumably has been successfully imitated in yeast. Mutation of Gln53 to Ser enhanced the selectivity for leucine residues in P1 without any obvious structural changes. Thus, amino acid 53 plays a crucial role in determining the cleavage preference of $\beta 5 \mathrm{c}$ and $\beta 5 \mathrm{i}$ subunits by modulating the interaction strength with 
Met45. Notably, this observation does probably not apply to subunit $\beta 5 \mathrm{t}$, as Thr cannot adopt diverse side chain conformations like Met45 and does not interact with Ala53, which is strictly conserved among $\beta 5 \mathrm{t}$ sequences.

Importantly, for all CP inhibition experiments the fluorogenic substrate Suc-Leu-Leu-ValTyr-AMC was used, irrespective of the mutations introduced in subunit y $\beta 5$. For a more correct determination of $\mathrm{IC}_{50}$ values specific fluorogenic peptide substrates for the subunits $\beta 5 \mathrm{c}, \beta 5 \mathrm{i}$ and $\beta 5 \mathrm{t}$ would be appropriate.

\subsection{Guidelines for the rational design of CP-selective inhibitors}

Various diseases, including autoimmune disorders and cancers, are characterized by elevated levels of inflammation signals and iCP subunits ${ }^{[22]}$. Hence, iCP-selective compounds constitute promising novel drugs for the treatment of autoimmune diseases. The iCP-selective inhibitor ONX 0914 was proven to halt disease progression in rheumatoid arthritis, experimental colitis, systemic lupus erythematosus and Hashimoto's thyroiditis by decreasing the levels of proinflammatory cytokines as well as autoantibodies and by modulating cytotoxic T cell responses ${ }^{[37,53]}$.

Previous studies demonstrated that peptide based inhibitors of the CP including ONX 0914 mimic the binding mode of natural substrates by forming an antiparallel $\beta$ sheet in the substrate binding channels of the active sites ${ }^{[13]}$. The herein presented crystal structures now elucidated that the binding mechanism of ONX 0914 to all active sites of the proteasome, the $\mathrm{c}$ and i subunits, is identical. Furthermore the selectivity of compounds solely depends on the potency of their electrophilic head group and the interactions of the ligand's P sites with the surrounding protein side chains. The exceptional importance of the interaction between the P1 site and the $\mathrm{S} 1$ pocket revealed by this study is corroborated by the nonpeptidic proteasome inhibitors salinosporamide A (marizomib) and omuralide that both occupy only the S1 site ${ }^{[13}$, 59]. Bearing in mind the uniform proteolytic mechanism of all active proteasome subunits, subunit-specific inhibitory compounds can only be developed by varying the side chains of peptidomimetics: $\beta 1 \mathrm{c}$ targeting compounds require acidic amino acids in their P1 site, while the hydrophobicity of the $\mathrm{S} 1$ pocket in $\beta 1 \mathrm{i}$ leads to a preference for branched hydrophobic residues, such as Val, Ile or Leu. Moreover, subunit $\beta 1$ i favours smaller and more polar amino acids in the $S 3$ pocket than subunit $\beta 1 \mathrm{c}$. Subunit $\beta 5 \mathrm{c}$ is perfectly suited to accommodate small apolar residues in P1, while subunit $\beta 5 \mathrm{i}$ is adapted for binding aromatic amino acids. The P3 site of subunit $\beta 5 \mathrm{c}$ accepts bulky hydrophobic side chains, whereas the P3 site of subunit $\beta 5 \mathrm{i}$ 
demands for small and preferentially polar residues. Since the S2 sites of the substrate binding channels are either shallow (subunit $\beta 1 \mathrm{c} / \mathrm{i}, \beta 2 \mathrm{c} / \mathrm{i}$ and $\beta 5 \mathrm{i}$ ) or even lacking $(\beta 5 \mathrm{c}$ ), the P2 positions can be occupied by spacious side chains of diverse chemical nature.

Ligand complex structures revealed that binding of the phenyl P1 side chain of ONX 0914 to subunit $\beta 5 \mathrm{c}$ is primarily disfavoured by sterical hindrance with Met45. Consistently, nonnatural derivatives of salinosporamide A and omuralide, bearing a phenyl moiety in the P1 site, demonstrate less affinity for the subunits $y \beta 5$ and $\beta 5 \mathrm{c}$ compared to their natural counterparts ${ }^{[119]}$. Besides ONX 0914, the epoxyketone PR-924 (Figure 7) represents another $\beta 5$ i-selective compound with phenylalanine as P1 side chain and alanine as P3 residue. However, unlike ONX 0914 and PR-924, the epoxyketone oprozomib (ONX 0912; Figure 6) that also bears a phenyl group as $\mathrm{P} 1$ residue is a potent inhibitor of both $\beta 5 \mathrm{c}$ and $\beta 5 \mathrm{i}$ with even slight preferences for the $\mathrm{c}$ subunit. The iCP crystal structure provides a plausible explanation for this observation. The methoxyserine of ONX 0912 fits into the S3 pocket of the $\beta 5 \mathrm{c}$ subunit formed by Ala27, but Ser27 in subunit $\beta 5$ i diminishes the size of the $\beta 5 \mathrm{i} 33$ pocket and thus, electrostatically and sterically hinders binding of ONX 0912 to $\beta 5 \mathrm{i}$. In this regard, even though the P1 residue appears to be the major determinant for the affinity of a ligand, the $\mathrm{P} 2$ and P3 residues also significantly contribute to the $\mathrm{IC}_{50}$ values. PR-825 (Figure 7) a structural analogue of ONX 0912 that possesses a leucine instead of a phenyl residue in P1, targets subunit $\beta 5 \mathrm{c}$ with 20 -fold selectivity over $\beta 5 \mathrm{i}$, demonstrating that leucine
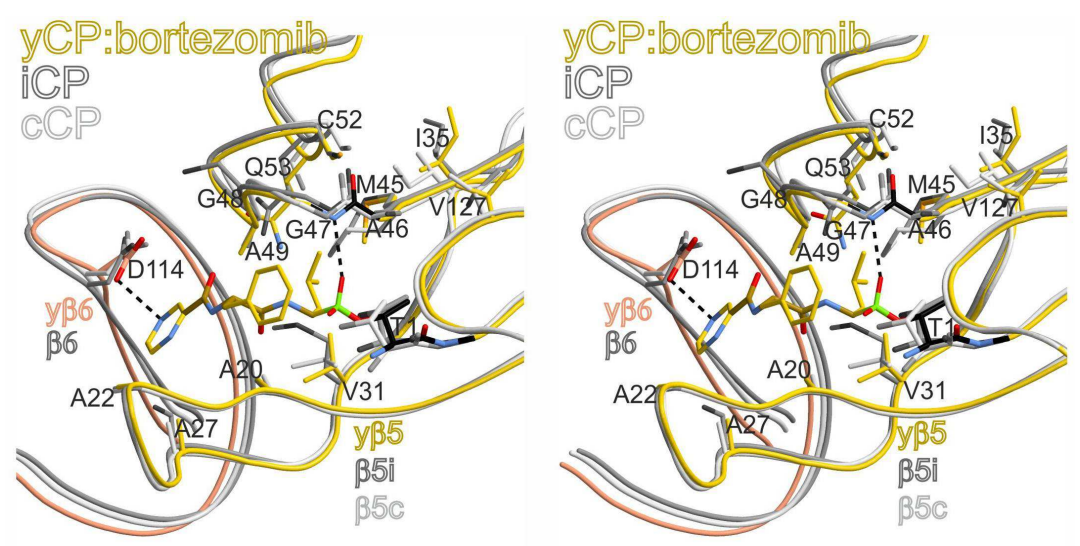

Figure 33 Stereo illustration of the yCP:bortezomib complex structure superimposed onto the $\mathrm{cCP}$ and iCP. Structural comparison of the bortezomib bound y $\beta 5$ subunit and the unliganded murine $\beta 5 \mathrm{c}$ and $\beta 5 \mathrm{i}$ active sites. Amino acids are labelled for the y $\beta 5$ subunit. Hydrogen bonds between bortezomib and the surrounding protein residues are indicated by black dashed lines.

Adapted from Huber et al., 2012 $2^{[32]}$.

and methoxyserine are suited to preferentially bind to the cCP. Carfilzomib, carrying a leucine side chain in both $\mathrm{P} 1$ and $\mathrm{P} 3$ sites is reported to be 5-times more selective for $\beta 5 \mathrm{c}$ than $\beta 5 \mathrm{i}^{[74]}$. 
Hence, leucine in $\mathrm{P} 1$ and leucine or methoxyserine in $\mathrm{P} 3$ give rise to $\beta 5 \mathrm{c}$ selectivity. Moreover, the $\mathrm{yCP}$ :bortezomib complex structure $^{[67]}$ together with the $\mathrm{cCP}$ and $\mathrm{iCP}$ coordinates elucidate that the P1 leucine and the P3 pyrazine ring of bortezomib fit into subunit $\beta 5 \mathrm{i}$ and $\beta 5 \mathrm{c}$ by interacting with Thr21, Ala22 and Ala27/Ser27 of $\beta 5 \mathrm{c} / \mathrm{i}$ and Asp144 of the neighbouring subunit $\beta 6$ (Figure 33). Hereby, bortezomib is capable of potently inhibiting both the $\mathrm{CCP}$ and $\mathrm{iCP}$.

Bearing in mind a sequence identity of more than $90 \%$ between murine and human subunits, the design of novel selective proteasome inhibitors for single CP-types and CP-subunits is now amenable. Such compounds enable the detailed examination of the biological impact of each active proteasome subunit and have huge medicinal potentials for the treatment of cancer and autoimmune diseases. 


\section{Bibliography}

[1] D. Finley (2009). Recognition and processing of ubiquitin-protein conjugates by the proteasome, Annu. Rev. Biochem., 78, 477-513.

[2] A. L. Goldberg, K. L. Rock (1992). Proteolysis, proteasomes and antigen presentation, Nature, 357, 375-379.

[3] a) J. D. Etlinger, A. L. Goldberg (1977). A soluble ATP-dependent proteolytic system responsible for the degradation of abnormal proteins in reticulocytes, Proc. Natl. Acad. Sci. U S A, 74, 54-58; b) A. Hershko, A. Ciechanover (1998). The ubiquitin system, Annu. Rev. Biochem., 67, 425-479.

[4] a) I. A. York, S. C. Chang, T. Saric, J. A. Keys, J. M. Favreau, A. L. Goldberg, K. L. Rock (2002). The ER aminopeptidase ERAP1 enhances or limits antigen presentation by trimming epitopes to 8-9 residues, Nat. Immunol., 3, 1177-1184; b) K. L. Rock, I.

A. York, A. L. Goldberg (2004). Post-proteasomal antigen processing for major histocompatibility complex class I presentation, Nat. Immunol., 5, 670-677.

[5] A. Townsend, J. Trowsdale (1993). The transporters associated with antigen presentation, Semin. Cell Biol., 4, 53-61.

[6] a) V. H. Engelhard (1994). Structure of peptides associated with MHC class I molecules, Curr. Opin. Immunol., 6, 13-23; b) K. Falk, O. Rotzschke, S. Stevanovic, G. Jung, H. G. Rammensee (1991). Allele-specific motifs revealed by sequencing of self-peptides eluted from MHC molecules, Nature, 351, 290-296.

[7] a) J. M. Vyas, A. G. Van der Veen, H. L. Ploegh (2008). The known unknowns of antigen processing and presentation, Nat. Rev. Immunol., 8, 607-618; b) J. Neefjes, M. L. Jongsma, P. Paul, O. Bakke (2011). Towards a systems understanding of MHC class I and MHC class II antigen presentation, Nat. Rev. Immunol., 11, 823-836.

[8] C. M. Pickart (2001). Mechanisms underlying ubiquitination, Annu. Rev. Biochem., 70, 503-533.

[9] J. M. T. Berg, J. L.; Stryer, L. (2002). Biochemistry, New York: W H Freeman.

[10] D. Voges, P. Zwickl, W. Baumeister (1999). The 26S proteasome: a molecular machine designed for controlled proteolysis, Annu. Rev. Biochem., 68, 1015-1068.

[11] M. Bochtler, L. Ditzel, M. Groll, R. Huber (1997). Crystal structure of heat shock locus V (HslV) from Escherichia coli, Proc. Natl. Acad. Sci. U S A, 94, 6070-6074.

[12] J. Löwe, D. Stock, B. Jap, P. Zwickl, W. Baumeister, R. Huber (1995). Crystal structure of the $20 \mathrm{~S}$ proteasome from the archaeon T. acidophilum at $3.4 \AA$ resolution, Science, 268, 533-539.

[13] M. Groll, L. Ditzel, J. Löwe, D. Stock, M. Bochtler, H. D. Bartunik, R. Huber (1997). Structure of 20S proteasome from yeast at $2.4 \AA$ resolution, Nature, 386, 463-471.

[14] P. Zwickl, A. Grziwa, G. Puhler, B. Dahlmann, F. Lottspeich, W. Baumeister (1992). Primary structure of the Thermoplasma proteasome and its implications for the structure, function, and evolution of the multicatalytic proteinase, Biochemistry, 31, 964-972.

[15] K. Tanaka, T. Yoshimura, T. Tamura, T. Fujiwara, A. Kumatori, A. Ichihara (1990). Possible mechanism of nuclear translocation of proteasomes, FEBS Lett., 271, 41-46.

[16] a) M. Groll, M. Bajorek, A. Köhler, L. Moroder, D. M. Rubin, R. Huber, M. H. Glickman, D. Finley (2000). A gated channel into the proteasome core particle, Nat. Struct. Biol., 7, 1062-1067; b) F. G. Whitby, E. I. Masters, L. Kramer, J. R. Knowlton, Y. Yao, C. C. Wang, C. P. Hill (2000). Structural basis for the activation of $20 \mathrm{~S}$ proteasomes by $11 \mathrm{~S}$ regulators, Nature, 408, 115-120.

[17] B. M. Stadtmueller, C. P. Hill (2011). Proteasome activators, Mol. Cell, 41, 8-19. 
[18] P. C. Ramos, J. Hockendorff, E. S. Johnson, A. Varshavsky, R. J. Dohmen (1998). Ump1p is required for proper maturation of the 20S proteasome and becomes its substrate upon completion of the assembly, Cell, 92, 489-499.

[19] M. Groll, W. Heinemeyer, S. Jäger, T. Ullrich, M. Bochtler, D. H. Wolf, R. Huber (1999). The catalytic sites of 20S proteasomes and their role in subunit maturation: a mutational and crystallographic study, Proc. Natl. Acad. Sci. U S A, 96, 10976-10983.

[20] L. Ditzel, R. Huber, K. Mann, W. Heinemeyer, D. H. Wolf, M. Groll (1998). Conformational constraints for protein self-cleavage in the proteasome, J. Mol. Biol., 279, 1187-1191.

[21] a) M. Unno, T. Mizushima, Y. Morimoto, Y. Tomisugi, K. Tanaka, N. Yasuoka, T. Tsukihara (2002). The structure of the mammalian 20S proteasome at $2.75 \AA$ resolution, Structure, 10, 609-618; b) M. Groettrup, R. Kraft, S. Kostka, S. Standera, R. Stohwasser, P. M. Kloetzel (1996). A third interferon-gamma-induced subunit exchange in the $20 \mathrm{~S}$ proteasome, Eur. J. Immunol., 26, 863-869; c) S. Murata, K. Sasaki, T. Kishimoto, S. Niwa, H. Hayashi, Y. Takahama, K. Tanaka (2007). Regulation of CD8+ T cell development by thymus-specific proteasomes, Science, 316, 1349-1353.

[22] E. M. Huber, M. Groll (2012). Inhibitors for the immuno- and constitutive proteasome: current and future trends in drug development, Angew. Chem. Int. Ed. Engl., 51, 8708-8720.

[23] M. Groettrup, C. J. Kirk, M. Basler (2010). Proteasomes in immune cells: more than peptide producers?, Nat. Rev. Immunol., 10, 73-78.

[24] M. Aki, N. Shimbara, M. Takashina, K. Akiyama, S. Kagawa, T. Tamura, N. Tanahashi, T. Yoshimura, K. Tanaka, A. Ichihara (1994). Interferon-gamma induces different subunit organizations and functional diversity of proteasomes, J. Biochem., $115,257-269$.

[25] T. A. Griffin, D. Nandi, M. Cruz, H. J. Fehling, L. V. Kaer, J. J. Monaco, R. A. Colbert (1998). Immunoproteasome assembly: cooperative incorporation of interferon gamma (IFN-gamma)-inducible subunits, J. Exp. Med., 187, 97-104.

[26] C. Realini, W. Dubiel, G. Pratt, K. Ferrell, M. Rechsteiner (1994). Molecular cloning and expression of a gamma-interferon-inducible activator of the multicatalytic protease, J. Biol. Chem., 269, 20727-20732.

[27] M. Rechsteiner, C. P. Hill (2005). Mobilizing the proteolytic machine: cell biological roles of proteasome activators and inhibitors, Trends Cell Biol., 15, 27-33.

[28] M. Rechsteiner, C. Realini, V. Ustrell (2000). The proteasome activator 11 S REG (PA28) and class I antigen presentation, Biochem. J., 345 Pt 1, 1-15.

[29] B. Guillaume, J. Chapiro, V. Stroobant, D. Colau, B. Van Holle, G. Parvizi, M. P. Bousquet-Dubouch, I. Theate, N. Parmentier, B. J. Van den Eynde (2010). Two abundant proteasome subtypes that uniquely process some antigens presented by HLA class I molecules, Proc. Natl. Acad. Sci. U S A, 107, 18599-18604.

[30] N. Klare, M. Seeger, K. Janek, P. R. Jungblut, B. Dahlmann (2007). Intermediate-type 20 S proteasomes in HeLa cells: "asymmetric" subunit composition, diversity and adaptation, J. Mol. Biol., 373, 1-10.

[31] M. Groll, M. Bochtler, H. Brandstetter, T. Clausen, R. Huber (2005). Molecular machines for protein degradation, Chembiochem, 6, 222-256.

[32] E. Huber, M. Basler, R. Schwab, W. Heinemeyer, C. J. Kirk, M. Groettrup, M. Groll (2012). Immuno- and constitutive proteasome crystal structures reveal differences in substrate and inhibitor specificity, Cell, 148, 727-738.

[33] a) C. Cardozo, R. A. Kohanski (1998). Altered properties of the branched chain amino acid-preferring activity contribute to increased cleavages after branched chain residues by the "immunoproteasome", J. Biol. Chem., 273, 16764-16770; b) M. Orlowski, C. 
Cardozo, C. Michaud (1993). Evidence for the presence of five distinct proteolytic components in the pituitary multicatalytic proteinase complex. Properties of two components cleaving bonds on the carboxyl side of branched chain and small neutral amino acids, Biochemistry, 32, 1563-1572.

[34] a) M. Gaczynska, K. L. Rock, A. L. Goldberg (1993). Gamma-interferon and expression of MHC genes regulate peptide hydrolysis by proteasomes, Nature, 365, 264-267; b) J. Driscoll, M. G. Brown, D. Finley, J. J. Monaco (1993). MHC-linked LMP gene products specifically alter peptidase activities of the proteasome, Nature, $365,262-264$.

[35] a) B. J. Van den Eynde, S. Morel (2001). Differential processing of class-I-restricted epitopes by the standard proteasome and the immunoproteasome, Curr. Opin.

Immunol., 13, 147-153; b) S. Murata, Y. Takahama, K. Tanaka (2008).

Thymoproteasome: probable role in generating positively selecting peptides, Curr. Opin. Immunol., 20, 192-196.

[36] E. Z. Kincaid, J. W. Che, I. York, H. Escobar, E. Reyes-Vargas, J. C. Delgado, R. M. Welsh, M. L. Karow, A. J. Murphy, D. M. Valenzuela, G. D. Yancopoulos, K. L. Rock (2011). Mice completely lacking immunoproteasomes show major changes in antigen presentation, Nat. Immunol., 13, 129-135.

[37] T. Muchamuel, M. Basler, M. A. Aujay, E. Suzuki, K. W. Kalim, C. Lauer, C. Sylvain, E. R. Ring, J. Shields, J. Jiang, P. Shwonek, F. Parlati, S. D. Demo, M. K. Bennett, C. J. Kirk, M. Groettrup (2009). A selective inhibitor of the immunoproteasome subunit LMP7 blocks cytokine production and attenuates progression of experimental arthritis, Nat. Med., 15, 781-787.

[38] U. Seifert, L. P. Bialy, F. Ebstein, D. Bech-Otschir, A. Voigt, F. Schroter, T. Prozorovski, N. Lange, J. Steffen, M. Rieger, U. Kuckelkorn, O. Aktas, P. M. Kloetzel, E. Kruger (2010). Immunoproteasomes preserve protein homeostasis upon interferon-induced oxidative stress, Cell, 142, 613-624.

[39] N. R. Gascoigne, E. Palmer (2011). Signaling in thymic selection, Curr. Opin. Immunol., 23, 207-212.

[40] M. J. Bevan (2007). Immunology. The cutting edge of T cell selection, Science, 316, 1291-1292.

[41] a) P. M. Voorhees, E. C. Dees, B. O'Neil, R. Z. Orlowski (2003). The proteasome as a target for cancer therapy, Clin. Cancer Res., 9, 6316-6325; b) L. R. Dick, P. E. Fleming (2010). Building on bortezomib: second-generation proteasome inhibitors as anti-cancer therapy, Drug Discov. Today, 15, 243-249.

[42] a) D. J. McConkey, K. Zhu (2008). Mechanisms of proteasome inhibitor action and resistance in cancer, Drug Resist. Updat., 11, 164-179; b) S. T. Nawrocki, J. S. Carew, K. Dunner, Jr., L. H. Boise, P. J. Chiao, P. Huang, J. L. Abbruzzese, D. J. McConkey (2005). Bortezomib inhibits PKR-like endoplasmic reticulum (ER) kinase and induces apoptosis via ER stress in human pancreatic cancer cells, Cancer Res., 65, 11510-

11519; c) E. A. Obeng, L. M. Carlson, D. M. Gutman, W. J. Harrington, Jr., K. P. Lee, L. H. Boise (2006). Proteasome inhibitors induce a terminal unfolded protein response in multiple myeloma cells, Blood, 107, 4907-4916; d) G. Bianchi, L. Oliva, P. Cascio, N. Pengo, F. Fontana, F. Cerruti, A. Orsi, E. Pasqualetto, A. Mezghrani, V. Calbi, G. Palladini, N. Giuliani, K. C. Anderson, R. Sitia, S. Cenci (2009). The proteasome load versus capacity balance determines apoptotic sensitivity of multiple myeloma cells to proteasome inhibition, Blood, 113, 3040-3049; e) T. Mujtaba, Q. P. Dou (2011). Advances in the understanding of mechanisms and therapeutic use of bortezomib, Discov. Med., 12, 471-480.

[43] I. Nickeleit, S. Zender, F. Sasse, R. Geffers, G. Brandes, I. Sorensen, H. Steinmetz, S. Kubicka, T. Carlomagno, D. Menche, I. Gutgemann, J. Buer, A. Gossler, M. P. 
Manns, M. Kalesse, R. Frank, N. P. Malek (2008). Argyrin a reveals a critical role for the tumor suppressor protein $\mathrm{p} 27$ (kip1) in mediating antitumor activities in response to proteasome inhibition, Cancer Cell, 14, 23-35.

[44] a) W. Lee, K. B. Kim (2011). The immunoproteasome: an emerging therapeutic target, Curr. Top. Med. Chem., 11, 2923-2930; b) Y. K. Ho, P. Bargagna-Mohan, M.

Wehenkel, R. Mohan, K. B. Kim (2007). LMP2-specific inhibitors: chemical genetic tools for proteasome biology, Chem. Biol., 14, 419-430.

[45] M. Mishto, E. Bellavista, A. Santoro, A. Stolzing, C. Ligorio, B. Nacmias, L. Spazzafumo, M. Chiappelli, F. Licastro, S. Sorbi, A. Pession, T. Ohm, T. Grune, C. Franceschi (2006). Immunoproteasome and LMP2 polymorphism in aged and Alzheimer's disease brains, Neurobiol. Aging, 27, 54-66.

[46] M. Diaz-Hernandez, F. Hernandez, E. Martin-Aparicio, P. Gomez-Ramos, M. A. Moran, J. G. Castano, I. Ferrer, J. Avila, J. J. Lucas (2003). Neuronal induction of the immunoproteasome in Huntington's disease, J. Neurosci., 23, 11653-11661.

[47] K. Puttaparthi, J. L. Elliott (2005). Non-neuronal induction of immunoproteasome subunits in an ALS model: possible mediation by cytokines, Exp. Neurol., 196, 441451.

[48] L. R. Fitzpatrick, J. S. Small, L. S. Poritz, K. J. McKenna, W. A. Koltun (2007). Enhanced intestinal expression of the proteasome subunit low molecular mass polypeptide 2 in patients with inflammatory bowel disease, Dis. Colon Rectum, 50, 337-348; discussion 348-350.

[49] T. Egerer, L. Martinez-Gamboa, A. Dankof, B. Stuhlmuller, T. Dorner, V. Krenn, K. Egerer, P. E. Rudolph, G. R. Burmester, E. Feist (2006). Tissue-specific up-regulation of the proteasome subunit beta5i (LMP7) in Sjogren's syndrome, Arthritis Rheum., 54, 1501-1508.

[50] a) I. Ferrer, B. Martin, J. G. Castano, J. J. Lucas, D. Moreno, M. Olive (2004). Proteasomal expression, induction of immunoproteasome subunits, and local MHC class I presentation in myofibrillar myopathy and inclusion body myositis, $\mathrm{J}$. Neuropathol. Exp. Neurol., 63, 484-498; b) Z. Yang, D. Gagarin, G. St Laurent, 3rd, N. Hammell, I. Toma, C. A. Hu, A. Iwasa, T. A. McCaffrey (2009). Cardiovascular inflammation and lesion cell apoptosis: a novel connection via the interferon-inducible immunoproteasome, Arterioscler. Thromb. Vasc. Biol., 29, 1213-1219.

[51] A. Visekruna, N. Slavova, S. Dullat, J. Grone, A. J. Kroesen, J. P. Ritz, H. J. Buhr, U. Steinhoff (2009). Expression of catalytic proteasome subunits in the gut of patients with Crohn's disease, Int. J. Colorectal. Dis., 24, 1133-1139.

[52] L. R. Fitzpatrick, V. Khare, J. S. Small, W. A. Koltun (2006). Dextran sulfate sodiuminduced colitis is associated with enhanced low molecular mass polypeptide 2 (LMP2) expression and is attenuated in LMP2 knockout mice, Dig. Dis. Sci., 51, 1269-1276.

[53] a) M. Basler, M. Dajee, C. Moll, M. Groettrup, C. J. Kirk (2010). Prevention of experimental colitis by a selective inhibitor of the immunoproteasome, J. Immunol., 185, 634-641; b) H. T. Ichikawa, T. Conley, T. Muchamuel, J. Jiang, S. Lee, T. Owen, J. Barnard, S. Nevarez, B. I. Goldman, C. J. Kirk, R. J. Looney, J. H. Anolik (2011). Novel proteasome inhibitors have a beneficial effect in murine lupus via the dual inhibition of type i interferon and autoantibody secreting cells, Arthritis Rheum., 64, 493-503; c) Y. Nagayama, M. Nakahara, M. Shimamura, I. Horie, K. Arima, N. Abiru (2012). Prophylactic and therapeutic efficacies of a selective inhibitor of the immunoproteasome for Hashimoto's thyroiditis, but not for Graves' hyperthyroidism, in mice, Clin. Exp. Immunol., 168, 268-273.

[54] a) D. J. Kuhn, S. A. Hunsucker, Q. Chen, P. M. Voorhees, M. Orlowski, R. Z. Orlowski (2009). Targeted inhibition of the immunoproteasome is a potent strategy against models of multiple myeloma that overcomes resistance to conventional drugs 
and nonspecific proteasome inhibitors, Blood, 113, 4667-4676; b) A. V. Singh, M. Bandi, M. A. Aujay, C. J. Kirk, D. E. Hark, N. Raje, D. Chauhan, K. C. Anderson (2011). PR-924, a selective inhibitor of the immunoproteasome subunit LMP-7, blocks multiple myeloma cell growth both in vitro and in vivo, Br. J. Haematol., 152, 155-163.

[55] F. Parlati, S. J. Lee, M. Aujay, E. Suzuki, K. Levitsky, J. B. Lorens, D. R. Micklem, P. Ruurs, C. Sylvain, Y. Lu, K. D. Shenk, M. K. Bennett (2009). Carfilzomib can induce tumor cell death through selective inhibition of the chymotrypsin-like activity of the proteasome, Blood, 114, 3439-3447.

[56] A. Vinitsky, C. Michaud, J. C. Powers, M. Orlowski (1992). Inhibition of the chymotrypsin-like activity of the pituitary multicatalytic proteinase complex, Biochemistry, 31, 9421-9428.

[57] M. Bogyo, J. S. McMaster, M. Gaczynska, D. Tortorella, A. L. Goldberg, H. Ploegh (1997). Covalent modification of the active site threonine of proteasomal beta subunits and the Escherichia coli homolog HslV by a new class of inhibitors, Proc. Natl. Acad. Sci. U S A, 94, 6629-6634.

[58] M. Groll, B. Schellenberg, A. S. Bachmann, C. R. Archer, R. Huber, T. K. Powell, S. Lindow, M. Kaiser, R. Dudler (2008). A plant pathogen virulence factor inhibits the eukaryotic proteasome by a novel mechanism, Nature, 452, 755-758.

[59] M. Groll, R. Huber, B. C. Potts (2006). Crystal structures of Salinosporamide A (NPI0052) and B (NPI-0047) in complex with the 20S proteasome reveal important consequences of beta-lactone ring opening and a mechanism for irreversible binding, J. Am. Chem. Soc., 128, 5136-5141.

[60] M. Groll, B. C. Potts (2011). Proteasome structure, function, and lessons learned from beta-lactone inhibitors, Curr. Top. Med. Chem., 11, 2850-2878.

[61] a) M. Groll, K. B. Kim, N. Kairies, R. Huber, C. M. Crews (2000). Crystal structure of epoxomicin: 20S proteasome reveals a molecular basis for selectivity of $\alpha^{\prime}, \beta^{\prime}-$ epoxyketone proteasome inhibitors, J. Am. Chem. Soc., 122, 1237-1238; b) K. B. Kim, J. Myung, N. Sin, C. M. Crews (1999). Proteasome inhibition by the natural products epoxomicin and dihydroeponemycin: insights into specificity and potency, Bioorg. Med. Chem. Lett., 9, 3335-3340.

[62] M. A. Grawert, N. Gallastegui, M. Stein, B. Schmidt, P. M. Kloetzel, R. Huber, M. Groll (2011). Elucidation of the alpha-keto-aldehyde binding mechanism: a lead structure motif for proteasome inhibition, Angew. Chem. Int. Ed. Engl., 50, 542-544.

[63] L. Borissenko, M. Groll (2007). 20S proteasome and its inhibitors: crystallographic knowledge for drug development, Chem. Rev., 107, 687-717.

[64] R. C. Kane, P. F. Bross, A. T. Farrell, R. Pazdur (2003). Velcade: U.S. FDA approval for the treatment of multiple myeloma progressing on prior therapy, Oncologist, 8 , 508-513.

[65] I. Tzvetanov, M. Spaggiari, J. Joseph, H. Jeon, J. Thielke, J. Oberholzer, E. Benedetti (2012). The use of bortezomib as a rescue treatment for acute antibody-mediated rejection: report of three cases and review of literature, Transplant. Proc., 44, 29712975.

[66] M. Escobar, M. Velez, A. Belalcazar, E. S. Santos, L. E. Raez (2011). The role of proteasome inhibition in nonsmall cell lung cancer, J. Biomed. Biotechnol., 2011, 806506.

[67] M. Groll, C. R. Berkers, H. L. Ploegh, H. Ovaa (2006). Crystal structure of the boronic acid-based proteasome inhibitor bortezomib in complex with the yeast $20 \mathrm{~S}$ proteasome, Structure, 14, 451-456.

[68] S. Arastu-Kapur, J. L. Anderl, M. Kraus, F. Parlati, K. D. Shenk, S. J. Lee, T. Muchamuel, M. K. Bennett, C. Driessen, A. J. Ball, C. J. Kirk (2011). 
Nonproteasomal targets of the proteasome inhibitors bortezomib and carfilzomib: a link to clinical adverse events, Clin. Cancer Res., 17, 2734-2743.

[69] P. Moreau, P. G. Richardson, M. Cavo, R. Z. Orlowski, J. F. San Miguel, A. Palumbo, J. L. Harousseau (2012). Proteasome inhibitors in multiple myeloma: 10 years later, Blood, 120, 947-959.

[70] S. Kumar, S. V. Rajkumar (2008). Many facets of bortezomib resistance/susceptibility, Blood, 112, 2177-2178.

[71] D. Chauhan, G. Li, R. Shringarpure, K. Podar, Y. Ohtake, T. Hideshima, K. C. Anderson (2003). Blockade of Hsp27 overcomes Bortezomib/proteasome inhibitor PS-341 resistance in lymphoma cells, Cancer Res., 63, 6174-6177.

[72] a) R. Oerlemans, N. E. Franke, Y. G. Assaraf, J. Cloos, I. van Zantwijk, C. R. Berkers, G. L. Scheffer, K. Debipersad, K. Vojtekova, C. Lemos, J. W. van der Heijden, B. Ylstra, G. J. Peters, G. L. Kaspers, B. A. Dijkmans, R. J. Scheper, G. Jansen (2008). Molecular basis of bortezomib resistance: proteasome subunit beta5 (PSMB5) gene mutation and overexpression of PSMB5 protein, Blood, 112, 2489-2499; b) S. Lu, J. Yang, Z. Chen, S. Gong, H. Zhou, X. Xu, J. Wang (2009). Different mutants of PSMB5 confer varying bortezomib resistance in T lymphoblastic lymphoma/leukemia cells derived from the Jurkat cell line, Exp. Hematol., 37, 831-837; c) N. E. Franke, D. Niewerth, Y. G. Assaraf, J. van Meerloo, K. Vojtekova, C. H. van Zantwijk, S. Zweegman, E. T. Chan, C. J. Kirk, D. P. Geerke, A. D. Schimmer, G. J. Kaspers, G. Jansen, J. Cloos (2011). Impaired bortezomib binding to mutant beta5 subunit of the proteasome is the underlying basis for bortezomib resistance in leukemia cells, Leukemia, 26, 757-768; d) E. Suzuki, S. Demo, E. Deu, J. Keats, S. Arastu-Kapur, P. L. Bergsagel, M. K. Bennett, C. J. Kirk (2011). Molecular mechanisms of bortezomib resistant adenocarcinoma cells, PLoS One, 6, e27996.

[73] K. Fostier, A. De Becker, R. Schots (2012). Carfilzomib: a novel treatment in relapsed and refractory multiple myeloma, OncoTargets Ther., 5, 237-244.

[74] S. D. Demo, C. J. Kirk, M. A. Aujay, T. J. Buchholz, M. Dajee, M. N. Ho, J. Jiang, G. J. Laidig, E. R. Lewis, F. Parlati, K. D. Shenk, M. S. Smyth, C. M. Sun, M. K. Vallone, T. M. Woo, C. J. Molineaux, M. K. Bennett (2007). Antitumor activity of PR-171, a novel irreversible inhibitor of the proteasome, Cancer Res., 67, 6383-6391.

[75] A. M. Ruschak, M. Slassi, L. E. Kay, A. D. Schimmer (2011). Novel proteasome inhibitors to overcome bortezomib resistance, J. Natl. Cancer Inst., 103, 1007-1017.

[76] H. J. Zhou, M. A. Aujay, M. K. Bennett, M. Dajee, S. D. Demo, Y. Fang, M. N. Ho, J. Jiang, C. J. Kirk, G. J. Laidig, E. R. Lewis, Y. Lu, T. Muchamuel, F. Parlati, E. Ring, K. D. Shenk, J. Shields, P. J. Shwonek, T. Stanton, C. M. Sun, C. Sylvain, T. M. Woo, J. Yang (2009). Design and synthesis of an orally bioavailable and selective peptide epoxyketone proteasome inhibitor (PR-047), J. Med. Chem, 52, 3028-3038.

[77] A. C. Mirabella, A. A. Pletnev, S. L. Downey, B. I. Florea, T. B. Shabaneh, M. Britton, M. Verdoes, D. V. Filippov, H. S. Overkleeft, A. F. Kisselev (2011). Specific cell-permeable inhibitor of proteasome trypsin-like sites selectively sensitizes myeloma cells to bortezomib and carfilzomib, Chem. Biol., 18, 608-618.

[78] K. W. Kalim, M. Basler, C. J. Kirk, M. Groettrup (2012). Immunoproteasome Subunit LMP7 Deficiency and Inhibition Suppresses Th1 and Th17 but Enhances Regulatory T Cell Differentiation, J. Immunol., 189, 4182-4193.

[79] W. O. Bullock, Fernandez, J. M., Short, J. M. (1987). XL1-Blue: A high efficiency plasmid transforming recA Escherichia coli strain with beta-galactosidase selection, BioTechniques, 5, 376-379.

[80] D. Hanahan (1983). Studies on transformation of Escherichia coli with plasmids, J. Mol. Biol., 166, 557-580. 
[81] W. Heinemeyer, A. Gruhler, V. Mohrle, Y. Mahe, D. H. Wolf (1993). PRE2, highly homologous to the human major histocompatibility complex-linked RING10 gene, codes for a yeast proteasome subunit necessary for chrymotryptic activity and degradation of ubiquitinated proteins, J. Biol. Chem., 268, 5115-5120.

[82] W. Heinemeyer, J. A. Kleinschmidt, J. Saidowsky, C. Escher, D. H. Wolf (1991). Proteinase yscE, the yeast proteasome/multicatalytic-multifunctional proteinase: mutants unravel its function in stress induced proteolysis and uncover its necessity for cell survival, EMBO J., 10, 555-562.

[83] W. Heinemeyer, M. Fischer, T. Krimmer, U. Stachon, D. H. Wolf (1997). The active sites of the eukaryotic $20 \mathrm{~S}$ proteasome and their involvement in subunit precursor processing, J. Biol. Chem., 272, 25200-25209.

[84] R. J. C. Estiveira (2008). The active subunits of the 20S Proteasome in Saccharomyces cerevisiae - Mutational analysis of their specificities and a C-terminal extension, $\mathrm{PhD}$ thesis, Universität Stuttgart.

[85] R. M. Esnouf (1997). An extensively modified version of MolScript that includes greatly enhanced coloring capabilities, J. Mol. Graph. Model., 15, 132-134, 112-133.

[86] Collaborative Computational Project (1994). The CCP4 suite: programs for protein crystallography, Acta Crystallogr. Sect. D - Biol. Crystallogr., 50, 760-763.

[87] P. Emsley, B. Lohkamp, W. G. Scott, K. Cowtan (2010). Features and development of Coot, Acta Crystallogr. Sect. D - Biol. Crystallogr., 66, 486-501.

[88] D. Turk (1992). Improvement of a Programme for Molecular Graphics and Manipulation of Electron Densities and Its Application for Protein Structure Determination, $\mathrm{PhD}$ thesis, Technische Universität München.

[89] P. J. Kraulis (1991). MOLSCRIPT: a program to produce both detailed and schematic plots of protein structures, J. Appl. Cryst., 24, 946-950.

[90] W. L. DeLano (2002). The PyMOL Molecular Graphics System, DeLano Scientific, San Carlos, CA, USA.

[91] SYBYL 8.0 Tripos International, 1699 South Hanley Rd., St. Louis, Missouri, 63144, USA

[92] W. Kabsch (2010). Xds, Acta Crystallogr. Sect. D - Biol. Crystallogr., 66, 125-132.

[93] E. Krissinel, Henrick, K. (2005). Detection of Protein Assemblies in Crystals, CompLife 2005, 3695, 163-174.

[94] K. Mullis, F. Faloona, S. Scharf, R. Saiki, G. Horn, H. Erlich (1986). Specific enzymatic amplification of DNA in vitro: the polymerase chain reaction, Cold Spring Harbor Symp. Quant. Biol., 51 Pt 1, 263-273.

[95] C. Papworth, Bauer, J. C., Braman, J. Wright, D. A. (1996). Site-directed mutagenesis in one day with $>80 \%$ efficiency, Strategies, 9, 3-4.

[96] W. J. Dower, J. F. Miller, C. W. Ragsdale (1988). High efficiency transformation of E. coli by high voltage electroporation, Nucleic Acids Res., 16, 6127-6145.

[97] R. D. Gietz, R. A. Woods (2002). Transformation of yeast by lithium acetate/singlestranded carrier DNA/polyethylene glycol method, Methods Enzymol., 350, 87-96.

[98] R. S. B. Sikorski, J. D. (1991). In vitro mutagenesis and plasmid shuffling from cloned gene to mutant yeast, Academic Press Inc., San Diego, 194, 302-318.

[99] F. Sanger, S. Nicklen, A. R. Coulson (1977). DNA sequencing with chain-terminating inhibitors, Proc. Natl. Acad. Sci. U S A, 74, 5463-5467.

[100] N. Gallastegui, M. Groll (2012). Analysing properties of proteasome inhibitors using kinetic and X-ray crystallographic studies, Methods Mol. Biol., 832, 373-390.

[101] G. Schmidtke, S. Emch, M. Groettrup, H. G. Holzhutter (2000). Evidence for the existence of a non-catalytic modifier site of peptide hydrolysis by the $20 \mathrm{~S}$ proteasome, J. Biol. Chem., 275, 22056-22063. 
[102] U. K. Laemmli (1970). Cleavage of structural proteins during the assembly of the head of bacteriophage T4, Nature, 227, 680-685.

[103] W. Kabsch (1993). Automatic processing of rotation diffraction data from crystals of initially unknown symmetry and cell constants, J. Appl. Cryst., 26, 795-800.

[104] A. J. McCoy, R. W. Grosse-Kunstleve, P. D. Adams, M. D. Winn, L. C. Storoni, R. J. Read (2007). Phaser crystallographic software, J. Appl. Cryst., 40, 658-674.

[105] M. Groll, R. Huber (2005). Purification, crystallization, and X-ray analysis of the yeast 20S proteasome, Methods Enzymol., 398, 329-336.

[106] A. A. Vagin, R. A. Steiner, A. A. Lebedev, L. Potterton, S. McNicholas, F. Long, G. N. Murshudov (2004). REFMAC5 dictionary: organization of prior chemical knowledge and guidelines for its use, Acta Crystallogr. Sect. D - Biol. Crystallogr., 60, 2184-2195.

[107] A. Nicholls, K. A. Sharp, B. Honig (1991). Protein folding and association: insights from the interfacial and thermodynamic properties of hydrocarbons, Proteins, 11, 281296.

[108] P. C. Ramos, A. J. Marques, M. K. London, R. J. Dohmen (2004). Role of C-terminal extensions of subunits beta 2 and beta7 in assembly and activity of eukaryotic proteasomes, J. Biol. Chem., 279, 14323-14330.

[109] a) M. A. Grawert, M. Groll (2012). Exploiting nature's rich source of proteasome inhibitors as starting points in drug development, Chem. Comm., 48, 1364-1378; b) P. Beck, C. Dubiella, M. Groll (2012). Covalent and non-covalent reversible proteasome inhibition, Biol. Chem., 393, 1101-1120.

[110] C. Blackburn, K. M. Gigstad, P. Hales, K. Garcia, M. Jones, F. J. Bruzzese, C. Barrett, J. X. Liu, T. A. Soucy, D. S. Sappal, N. Bump, E. J. Olhava, P. Fleming, L. R. Dick, C. Tsu, M. D. Sintchak, J. L. Blank (2010). Characterization of a new series of noncovalent proteasome inhibitors with exquisite potency and selectivity for the $20 \mathrm{~S}$ beta5-subunit, Biochem. J., 430, 461-476.

[111] H. G. Rammensee, T. Friede, S. Stevanovi (1995). MHC ligands and peptide motifs: first listing, Immunogenetics, 41, 178-228.

[112] W. Chen, C. C. Norbury, Y. Cho, J. W. Yewdell, J. R. Bennink (2001). Immunoproteasomes shape immunodominance hierarchies of antiviral CD8(+) T cells at the levels of T cell repertoire and presentation of viral antigens, J. Exp. Med., 193, 1319-1326.

[113] P. Deol, D. M. Zaiss, J. J. Monaco, A. J. Sijts (2007). Rates of processing determine the immunogenicity of immunoproteasome-generated epitopes, J. Immunol., 178, 7557-7562.

[114] R. E. Toes, A. K. Nussbaum, S. Degermann, M. Schirle, N. P. Emmerich, M. Kraft, C. Laplace, A. Zwinderman, T. P. Dick, J. Muller, B. Schonfisch, C. Schmid, H. J. Fehling, S. Stevanovic, H. G. Rammensee, H. Schild (2001). Discrete cleavage motifs of constitutive and immunoproteasomes revealed by quantitative analysis of cleavage products, J. Exp. Med., 194, 1-12.

[115] M. Gaczynska, K. L. Rock, T. Spies, A. L. Goldberg (1994). Peptidase activities of proteasomes are differentially regulated by the major histocompatibility complexencoded genes for LMP2 and LMP7, Proc. Natl. Acad. Sci. U S A, 91, 9213-9217.

[116] H. J. Fehling, W. Swat, C. Laplace, R. Kuhn, K. Rajewsky, U. Muller, H. von Boehmer (1994). MHC class I expression in mice lacking the proteasome subunit LMP-7, Science, 265, 1234-1237.

[117] L. Tu, C. Moriya, T. Imai, H. Ishida, K. Tetsutani, X. Duan, S. Murata, K. Tanaka, C. Shimokawa, H. Hisaeda, K. Himeno (2009). Critical role for the immunoproteasome subunit LMP7 in the resistance of mice to Toxoplasma gondii infection, Eur. J. Immunol., 39, 3385-3394. 
[118] a) L. Van Kaer, P. G. Ashton-Rickardt, M. Eichelberger, M. Gaczynska, K. Nagashima, K. L. Rock, A. L. Goldberg, P. C. Doherty, S. Tonegawa (1994). Altered peptidase and viral-specific T cell response in LMP2 mutant mice, Immunity, 1, 533541; b) M. Basler, J. Moebius, L. Elenich, M. Groettrup, J. J. Monaco (2006). An altered T cell repertoire in MECL-1-deficient mice, J. Immunol., 176, 6665-6672.

[119] a) E. J. Corey, W. D. Li (1999). Total synthesis and biological activity of lactacystin, omuralide and analogs, Chem. Pharm. Bull., 47, 1-10; b) M. Nett, T. A. Gulder, A. J. Kale, C. C. Hughes, B. S. Moore (2009). Function-oriented biosynthesis of betalactone proteasome inhibitors in Salinispora tropica, J. Med. Chem., 52, 6163-6167. 


\section{Appendix}

\begin{tabular}{|c|c|c|c|c|c|c|c|c|c|c|}
\hline \multicolumn{2}{|c|}{ Subunit } & \multicolumn{3}{|c|}{$i C P$} & \multicolumn{3}{|c|}{$c \boldsymbol{C P}$} & \multicolumn{3}{|c|}{ iCP compared to $c C P$} \\
\hline 1 & 2 & $\begin{array}{c}\text { Interface } \\
{\left[\AA^{2}\right]}\end{array}$ & $h b$ & $s b$ & $\begin{array}{c}\text { Interface } \\
{\left[\AA^{2}\right]}\end{array}$ & $h b$ & $s b$ & $\begin{array}{c}\text { Interface } \\
{[\%]}\end{array}$ & $\Delta h b$ & $\Delta s b$ \\
\hline$\alpha 1$ & $\alpha 2$ & 1399.9 & 18 & 3 & 1403.3 & 17 & 3 & 99.8 & +1 & 0 \\
\hline$\alpha 1$ & $\alpha 7$ & 1323.6 & 19 & 4 & 1341.2 & 16 & 3 & 98.7 & +3 & +1 \\
\hline$\alpha 2$ & $\alpha 3$ & 1459.1 & 16 & 5 & 1462.1 & 18 & 5 & 99.8 & -2 & 0 \\
\hline$\alpha 3$ & $\alpha 4$ & 1430.3 & 20 & 5 & 1500.0 & 18 & 6 & 95.4 & +2 & -1 \\
\hline$\alpha 4$ & $\alpha 5$ & 1395.6 & 21 & 4 & 1423.8 & 25 & 9 & 98.0 & -4 & -5 \\
\hline$\alpha 5$ & $\alpha 6$ & 1327.8 & 19 & 4 & 1336.7 & 18 & 4 & 99.3 & +1 & 0 \\
\hline$\alpha 6$ & $\alpha 7$ & 1367.0 & 19 & 1 & 1365.4 & 20 & 2 & 100.1 & -1 & -1 \\
\hline$\beta 1(\mathrm{i})$ & $\beta 1(\mathrm{i})^{6}$ & 750.8 & 8 & 6 & 945.7 & 8 & 8 & 79.4 & 0 & -2 \\
\hline$\beta 1(\mathrm{i})$ & $\beta 2(\mathrm{i})$ & 471.6 & 5 & 7 & 552.8 & 8 & 5 & 85.3 & -3 & +2 \\
\hline$\beta 1(\mathrm{i})$ & $\beta 7$ & 717.4 & 11 & 8 & 715.7 & 13 & 8 & 100.2 & -2 & 0 \\
\hline$\beta 1(\mathrm{i})^{\mathrm{c}}$ & $\beta 7$ & 1110.7 & 16 & 3 & 1142.4 & 19 & 2 & 97.2 & -3 & +1 \\
\hline$\beta 2(\mathrm{i})$ & $\beta 3$ & 1531.0 & 25 & 12 & 1601.7 & 25 & 11 & 95.6 & 0 & +1 \\
\hline$\beta 2(\mathrm{i})$ & $\beta 7^{\circ}$ & 694.4 & 8 & 11 & 744.0 & 7 & 7 & 93.3 & +1 & +4 \\
\hline$\beta 3$ & $\beta 4$ & 752.1 & 12 & 4 & 737.6 & 13 & 5 & 102.0 & -1 & -1 \\
\hline$\beta 3$ & $\beta 5(\mathrm{i})^{\circ}$ & 855.7 & 19 & 8 & 841.6 & 19 & 8 & 101.7 & 0 & 0 \\
\hline$\beta 4$ & $\beta 4^{\prime}$ & 734.0 & 14 & 0 & 761.7 & 11 & 0 & 96.4 & +3 & 0 \\
\hline$\beta 4$ & $\beta 5(\mathrm{i})$ & 589.4 & 3 & 5 & 590.0 & 4 & 4 & 99.9 & -1 & +1 \\
\hline$\beta 4^{\prime}$ & $\beta 5(\mathrm{i})$ & 597.0 & 8 & 7 & 654.3 & 7 & 10 & 91.2 & +1 & -3 \\
\hline$\beta 5(\mathrm{i})$ & $\beta 6$ & 688.7 & 12 & 4 & 547.2 & 10 & 4 & 125.9 & +2 & 0 \\
\hline$\beta 6$ & $\beta 7$ & 970.8 & 13 & 1 & 974.3 & 13 & 3 & 99.6 & 0 & -2 \\
\hline$\beta 6^{\circ}$ & $\beta 2(i)$ & 1130.6 & 15 & 4 & 1215.8 & 16 & 5 & 93.0 & -1 & -1 \\
\hline$\beta 6^{6}$ & $\beta 3$ & 657.6 & 8 & 5 & 673.8 & 10 & 6 & 97.6 & -2 & -1 \\
\hline$\beta 1(\mathrm{i})$ & $\alpha 1$ & 457.4 & 5 & 3 & 417.0 & 3 & 0 & 109.7 & +2 & +3 \\
\hline$\beta 1(\mathrm{i})$ & $\alpha 7$ & 457.8 & 7 & 3 & 440.7 & 2 & 1 & 103.9 & +5 & +2 \\
\hline$\beta 2(\mathrm{i})$ & $\alpha 1$ & 566.2 & 8 & 6 & 583.5 & 7 & 5 & 97.0 & +1 & +1 \\
\hline$\beta 2(\mathrm{i})$ & $\alpha 2$ & 441.8 & 3 & 0 & 463.3 & 5 & 0 & 95.4 & -2 & 0 \\
\hline$\beta 3$ & $\alpha 2$ & 412.6 & 8 & 6 & 427.7 & 10 & 6 & 96.5 & -2 & 0 \\
\hline
\end{tabular}

Table 15 PISA analysis ${ }^{[93]}$ of interface areas and interactions of adjacent proteasome subunits in the murine cCP and iCP. The number of hydrogen bonds (hb) and salt bridges (sb) as well as their differences between $\mathrm{cCP}$ and $\mathrm{iCP}(\Delta)$ are provided. Interface areas of iCP subunits are given as percentage of the corresponding values in the $\mathrm{cCP}$. 


\begin{tabular}{|c|c|c|c|}
\hline & $\begin{array}{l}y \boldsymbol{C P} \\
y \beta 5 Q 53 S\end{array}$ & $\begin{array}{l}\boldsymbol{y} \boldsymbol{C P} \\
y \beta 5 Q 53 \text { S:bortezomib }\end{array}$ & $\begin{array}{l}\boldsymbol{y} \boldsymbol{C P} \\
y \beta 5 Q 53 S: O N X 0914\end{array}$ \\
\hline \multicolumn{4}{|l|}{ Crystal parameters } \\
\hline Space group & $\mathrm{P} 2_{1}$ & $\mathrm{P} 2_{1}$ & $\mathrm{P} 2_{1}$ \\
\hline \multirow[t]{4}{*}{ Cell constants } & $a=134.7 \AA$ & $a=136.3 \AA$ & $a=136.7 \AA$ \\
\hline & $\mathrm{b}=301.9 \AA$ & $\mathrm{b}=301.4 \AA$ & $\mathrm{b}=301.5 \AA$ \\
\hline & $\mathrm{c}=144.7 \AA$ & $\mathrm{c}=145.2 \AA$ & $\mathrm{c}=145.2 \AA$ \\
\hline & $\beta=112.9^{\circ}$ & $\beta=113.1^{\circ}$ & $\beta=112.8^{\circ}$ \\
\hline $\mathrm{CPs} / \mathrm{AU}^{\mathrm{a}}$ & 1 & 1 & 1 \\
\hline \multicolumn{4}{|l|}{ Data collection } \\
\hline Beam line & X06SA, SLS & X06SA, SLS & X06SA, SLS \\
\hline Wavelength $(\AA)$ & 1.0 & 1.0 & 1.0 \\
\hline \multirow[t]{2}{*}{ Resolution range $(\AA)^{\mathrm{b}}$} & $49-2.9$ & $49-3.0$ & $49-3.4$ \\
\hline & $(3.0-2.9)$ & $(3.1-3.0)$ & $(3.5-3.4)$ \\
\hline No. observations & 717696 & 653661 & 521471 \\
\hline No. unique reflections ${ }^{c}$ & 230488 & 209442 & 146379 \\
\hline Completeness $(\%)^{\mathrm{b}}$ & $98.2(98.6)$ & $97.5(99.1)$ & $98.6(99.0)$ \\
\hline $\mathrm{R}_{\text {merge }}(\%)^{\mathrm{b}, \mathrm{d}}$ & $7.9(55.3)$ & $8.4(62.0)$ & $13.6(58.7)$ \\
\hline $\mathrm{I} / \sigma(\mathrm{I})^{\mathrm{b}}$ & $11.8(2.6)$ & $13.0(3.2)$ & $7.6(2.3)$ \\
\hline \multicolumn{4}{|l|}{ Refinement (REFMAC5) } \\
\hline Resolution range $(\AA)$ & $15-2.9$ & $15-3.0$ & $15-3.4$ \\
\hline No. refl. working set & 218963 & 308271 & 176276 \\
\hline No. refl. test set & 10948 & 15413 & 8813 \\
\hline No. non hydrogen & 50864 & 51032 & 51116 \\
\hline No. of ligand atoms & - & 168 & 294 \\
\hline Water molecules & 1322 & 1322 & 1322 \\
\hline $\mathrm{R}_{\text {work }} / \mathrm{R}_{\text {free }}(\%)^{\mathrm{e}}$ & $15.1 / 21.1$ & $14.7 / 20.6$ & $14.2 / 21.7$ \\
\hline r.m.s.d. bond $(\AA) /\left({ }^{\circ}\right)^{f}$ & $0.011 / 1.599$ & $0.012 / 1.637$ & $0.011 / 1.591$ \\
\hline Average B-factor $\left(\AA^{2}\right)$ & 61.7 & 64.5 & 70.7 \\
\hline Ramachandran Plot $(\%)^{\mathrm{g}}$ & $94.3 / 4.7 / 1.0$ & $94.4 / 4.9 / 0.7$ & $92.3 / 6.6 / 1.1$ \\
\hline
\end{tabular}

Table $16 \mathrm{X}$-ray data collection and refinement statistics of structures of the $\mathrm{yCP}$ mutant $\mathrm{y \beta 5Q53S}$.

a Asymmetric unit.

b The values in parentheses of resolution range, completeness, $R_{\text {merge }}$ and $I / \sigma$ (I) correspond to the last resolution shell.

${ }^{c}$ Friedel pairs were treated as identical reflections.

${ }^{\mathrm{d}} \mathrm{R}_{\text {merge }}(\mathrm{I})=\Sigma_{\mathrm{hkl}} \Sigma_{\mathrm{j}}\left|\left[\mathrm{I}(h k l)_{\mathrm{j}}-\mathrm{I}(h k l)\right]\right| \Sigma_{\mathrm{hkl}} \mathrm{I}_{\mathrm{hkl}}$, where $\mathrm{I}(\mathrm{hkl})_{\mathrm{j}}$ is the $\mathrm{j}^{\text {th }}$ measurement of the intensity of reflection hkl and $\langle\mathrm{I}(\mathrm{hkl})\rangle$ is the average intensity.

e $\mathrm{R}=\Sigma_{\mathrm{hkl}}|| \mathrm{F}_{\mathrm{obs}}|-| \mathrm{F}_{\text {calc }}|| \Sigma_{\mathrm{hkl}}\left|\mathrm{F}_{\mathrm{obs}}\right|$, where $\mathrm{R}_{\text {free }}$ is calculated for a randomly chosen $5 \%$ of reflections, which were not used for structure refinement, and $\mathrm{R}_{\text {work }}$ is calculated for the remaining reflections.

${ }^{f}$ Deviations from ideal bond lengths/angles.

${ }^{\mathrm{g}}$ Number of residues in favoured, allowed or outlier region. 


\begin{tabular}{|c|c|c|c|}
\hline & $\begin{array}{l}\text { yCP } \\
y \beta 5 \text { A27S K32N A46S } \\
T 57 R \text { G48C K71G } \\
V 127 T\end{array}$ & $\begin{array}{l}\text { yCP } \\
y \beta 5 \text { A27S K32N A46S } \\
\text { T57R G48C K71G } \\
\text { V127T:bortezomib }\end{array}$ & $\begin{array}{l}y \boldsymbol{y} \boldsymbol{P P} \\
y \beta 5 \text { A27S K32N A46S } \\
\text { T57R G48C K71G } \\
\text { V127T:ONX 0914 }\end{array}$ \\
\hline \multicolumn{4}{|l|}{ Crystal parameters } \\
\hline Space group & $\mathrm{P} 2_{1}$ & $\mathrm{P} 2_{1}$ & $\mathrm{P} 2_{1}$ \\
\hline Cell constants & $\mathrm{a}=134.5 \AA$ & $a=136.5 \AA$ & $a=136.6 \AA$ \\
\hline & $\mathrm{b}=301.0 \AA$ & $\mathrm{b}=300.4 \AA$ & $\mathrm{b}=300.3 \AA$ \\
\hline & $\mathrm{c}=144.4 \AA$ & $c=145.6 \AA$ & $\mathrm{c}=145.8 \AA$ \\
\hline & $\beta=113.0^{\circ}$ & $\beta=113.1^{\circ}$ & $\beta=113.2^{\circ}$ \\
\hline $\mathrm{CPs} / \mathrm{AU}^{\mathrm{a}}$ & 1 & 1 & 1 \\
\hline \multicolumn{4}{|l|}{ Data collection } \\
\hline Beam line & X06SA, SLS & X06SA, SLS & X06SA, SLS \\
\hline Wavelength $(\AA)$ & 1.0 & 1.0 & 1.0 \\
\hline \multirow[t]{2}{*}{ Resolution range $(\AA)^{b}$} & $49-2.5$ & $49-2.8$ & $49-2.6$ \\
\hline & $(2.6-2.5)$ & $(2.9-2.8)$ & $(2.7-2.6)$ \\
\hline No. observations & 1048068 & 813713 & 1001216 \\
\hline No. unique reflections ${ }^{c}$ & 353449 & 260234 & 324245 \\
\hline Completeness $(\%)^{\mathrm{b}}$ & $97.3(98.4)$ & $98.5(99.0)$ & $98.3(99.4)$ \\
\hline $\mathrm{R}_{\text {merge }}(\%)^{\mathrm{b}, \mathrm{d}}$ & $7.5(59.7)$ & $7.5(46.2)$ & $7.6(56.0)$ \\
\hline $\mathrm{I} / \sigma(\mathrm{I})^{\mathrm{b}}$ & $9.2(2.0)$ & $11.9(3.4)$ & $10.8(2.7)$ \\
\hline \multicolumn{4}{|l|}{$\begin{array}{l}\text { Refinement } \\
\text { (REFMAC5) }\end{array}$} \\
\hline Resolution range $(\AA)$ & $15-2.5$ & $15-2.8$ & $15-2.6$ \\
\hline No. refl. working set & 335776 & 247222 & 308031 \\
\hline No. refl. test set & 16788 & 12361 & 15401 \\
\hline No. non hydrogen & 50888 & 51047 & 51135 \\
\hline No. of ligand atoms & - & 168 & 294 \\
\hline Water molecules & 1340 & 1340 & 1340 \\
\hline $\mathrm{R}_{\text {work }} / \mathrm{R}_{\text {free }}(\%)^{\mathrm{e}}$ & $19.0 / 23.6$ & $15.5 / 21.0$ & $16.9 / 21.5$ \\
\hline r.m.s.d. bond $(\AA) /\left({ }^{\circ}\right)^{\mathrm{f}}$ & $0.012 / 1.652$ & $0.011 / 1.551$ & $0.012 / 1.649$ \\
\hline Average B-factor $\left(\AA^{2}\right)$ & 58.1 & 62.7 & 62.1 \\
\hline Ramachandran Plot $(\%)^{\mathrm{g}}$ & $94.4 / 4.8 / 0.8$ & $95.5 / 3.7 / 0.7$ & $95.6 / 3.8 / 0.6$ \\
\hline
\end{tabular}

Table $17 \mathrm{X}$-ray data collection and refinement statistics of structures of the yCP mutant yß5 A27S K32N A46S T57R G48C K71G V127T.

a Asymmetric unit.

b The values in parentheses of resolution range, completeness, $R_{\text {merge }}$ and $I / \sigma$ (I) correspond to the last resolution shell.

c Friedel pairs were treated as identical reflections.

${ }^{d} R_{\text {merge }}(\mathrm{I})=\Sigma_{\mathrm{hkl}} \Sigma_{\mathrm{j}}\left|\left[\mathrm{I}(h k l)_{\mathrm{j}}-\mathrm{I}(h k l)\right]\right| \Sigma_{\mathrm{hkl}} \mathrm{I}_{\mathrm{hkl}}$, where $\mathrm{I}(\mathrm{hkl})_{\mathrm{j}}$ is the $\mathrm{j}^{\text {th }}$ measurement of the intensity of reflection hkl and $\langle\mathrm{I}(\mathrm{hkl})\rangle$ is the average intensity.

e $\mathrm{R}=\Sigma_{\mathrm{hkl}}|| \mathrm{F}_{\mathrm{obs}}|-| \mathrm{F}_{\text {calc }}|| / \Sigma_{\mathrm{hkl}}\left|\mathrm{F}_{\mathrm{obs}}\right|$, where $\mathrm{R}_{\text {free }}$ is calculated for a randomly chosen $5 \%$ of reflections, which were not used for structure refinement, and $\mathrm{R}_{\text {work }}$ is calculated for the remaining reflections.

$\mathrm{f}$ Deviations from ideal bond lengths/angles.

$\mathrm{g}$ Number of residues in favoured, allowed or outlier region. 


\begin{tabular}{|c|c|c|c|}
\hline & $\begin{array}{l}\boldsymbol{y C P} \\
y \beta 5 M 45 R\end{array}$ & $\begin{array}{l}\boldsymbol{y C P} \\
y \beta 5 \text { M45R:bortezomib }\end{array}$ & $\begin{array}{l}\boldsymbol{y} \boldsymbol{C P} \\
y \beta 5 \text { M45R:ONX } 0914\end{array}$ \\
\hline \multicolumn{4}{|l|}{ Crystal parameters } \\
\hline Space group & $\mathrm{P} 2_{1}$ & $\mathrm{P} 2_{1}$ & $\mathrm{P} 2_{1}$ \\
\hline \multirow[t]{4}{*}{ Cell constants } & $\mathrm{a}=134.6 \AA$ & $\mathrm{a}=136.0 \AA$ & $a=135.9 \AA$ \\
\hline & $\mathrm{b}=302.8 \AA$ & $\mathrm{b}=300.3 \AA$ & $\mathrm{b}=298.1 \AA$ \\
\hline & $\mathrm{c}=145.2 \AA$ & $\mathrm{c}=145.1 \AA$ & $\mathrm{c}=144.4 \AA$ \\
\hline & $\beta=112.7^{\circ}$ & $\beta=112.8^{\circ}$ & $\beta=112.7^{\circ}$ \\
\hline $\mathrm{CPs} / \mathrm{AU}^{\mathrm{a}}$ & 1 & 1 & 1 \\
\hline \multicolumn{4}{|l|}{ Data collection } \\
\hline Beam line & X06SA, SLS & X06SA, SLS & X06SA, SLS \\
\hline Wavelength $(\AA)$ & 1.0 & 1.0 & 1.0 \\
\hline \multirow[t]{2}{*}{ Resolution range $(\AA)^{\mathrm{b}}$} & $49-3.0$ & $49-2.9$ & $48-2.8$ \\
\hline & $(3.1-3.0)$ & $(3.0-2.9)$ & $(2.9-2.8)$ \\
\hline No. observations & 640110 & 700484 & 747601 \\
\hline No. unique reflections ${ }^{c}$ & 208693 & 230999 & 248239 \\
\hline Completeness $(\%)^{\mathrm{b}}$ & $97.7(98.9)$ & $97.7(98.6)$ & $95.6(96.1)$ \\
\hline $\mathrm{R}_{\text {merge }}(\%)^{\mathrm{b}, \mathrm{d}}$ & $10.6(49.4)$ & $8.7(55.5)$ & $7.4(50.6)$ \\
\hline $\mathrm{I} / \sigma(\mathrm{I})^{\mathrm{b}}$ & $8.7(2.7)$ & $10.1(1.7)$ & $11.1(1.8)$ \\
\hline \multicolumn{4}{|l|}{ Refinement (REFMAC5) } \\
\hline Resolution range $(\AA)$ & $15-3.0$ & $15-2.9$ & $15-2.8$ \\
\hline No. refl. working set & 198258 & 219448 & 235827 \\
\hline No. refl. test set & 9912 & 10972 & 11791 \\
\hline No. non hydrogen & 50894 & 51053 & 51137 \\
\hline No. of ligand atoms (ligand; MES) & - & 168 & 318 \\
\hline Water molecules & 1340 & 1340 & 1340 \\
\hline $\mathrm{R}_{\text {work }} / \mathrm{R}_{\text {free }}(\%)^{\mathrm{e}}$ & $14.8 / 20.7$ & $15.9 / 21.7$ & $17.5 / 23.9$ \\
\hline r.m.s.d. bond $(\AA) /\left({ }^{\circ}\right)^{f}$ & $0.014 / 1.802$ & $0.012 / 1.658$ & $0.014 / 1.830$ \\
\hline Average B-factor $\left(\AA^{2}\right)$ & 59.2 & 61.0 & 64.1 \\
\hline Ramachandran Plot $(\%)^{\mathrm{g}}$ & $94.2 / 5.1 / 0.8$ & $95.1 / 4.2 / 0.8$ & $93.7 / 5.4 / 0.8$ \\
\hline
\end{tabular}

Table 18 X-ray data collection and refinement statistics of structures of the yCP mutant y $\beta 5$ M45R.

a Asymmetric unit.

b The values in parentheses of resolution range, completeness, $\mathrm{R}_{\text {merge }}$ and $\mathrm{I} / \sigma$ (I) correspond to the last resolution shell.

c Friedel pairs were treated as identical reflections.

${ }^{\mathrm{d}} \mathrm{R}_{\text {merge }}(\mathrm{I})=\Sigma_{\mathrm{hkl}} \Sigma_{\mathrm{j}}\left|\left[\mathrm{I}(h k l)_{\mathrm{j}}-\mathrm{I}(h k l)\right]\right| \Sigma_{\mathrm{hkl}} \mathrm{I}_{\mathrm{hkl}}$, where $\mathrm{I}(\mathrm{hkl})_{\mathrm{j}}$ is the $\mathrm{j}^{\text {th }}$ measurement of the intensity of reflection hkl and $\langle\mathrm{I}(\mathrm{hkl})\rangle$ is the average intensity.

e $\mathrm{R}=\Sigma_{\mathrm{hkl}}|| \mathrm{F}_{\mathrm{obs}}|-| \mathrm{F}_{\text {calc }}|| / \Sigma_{\mathrm{hkl}}\left|\mathrm{F}_{\mathrm{obs}}\right|$, where $\mathrm{R}_{\text {free }}$ is calculated for a randomly chosen $5 \%$ of reflections, which were not used for structure refinement, and $\mathrm{R}_{\mathrm{work}}$ is calculated for the remaining reflections.

$\mathrm{f}$ Deviations from ideal bond lengths/angles.

$\mathrm{g}$ Number of residues in favoured, allowed or outlier region. 


\begin{tabular}{|c|c|c|c|}
\hline & $\begin{array}{l}\boldsymbol{y C P} \\
y \beta 5 \text { I35T M45R }\end{array}$ & $\begin{array}{l}y \boldsymbol{C P} \\
y \beta 5 \text { I35T M45R:bortezomib }\end{array}$ & $\begin{array}{l}\boldsymbol{y} \boldsymbol{C P} \\
y \beta 5 \text { I35T M45R:ONX } 0914\end{array}$ \\
\hline \multicolumn{4}{|l|}{ Crystal parameters } \\
\hline Space group & $\mathrm{P} 2_{1}$ & $\mathrm{P} 2_{1}$ & $\mathrm{P} 2_{1}$ \\
\hline Cell constants & $a=133.9 \AA$ & $\mathrm{a}=135.1 \AA$ & $a=135.5 \AA$ \\
\hline & $\mathrm{b}=300.6 \AA$ & $\mathrm{b}=301.0 \AA$ & $\mathrm{b}=299.1 \AA$ \\
\hline & $c=144.2 \AA$ & $c=146.1 \AA$ & $c=145.7 \AA$ \\
\hline & $\beta=112.8^{\circ}$ & $\beta=112.8^{\circ}$ & $\beta=112.9^{\circ}$ \\
\hline $\mathrm{CPs} / \mathrm{AU}^{\mathrm{a}}$ & 1 & 1 & 1 \\
\hline \multicolumn{4}{|l|}{ Data collection } \\
\hline Beam line & X06SA, SLS & X06SA, SLS & X06SA, SLS \\
\hline Wavelength $(\AA)$ & 1.0 & 1.0 & 1.0 \\
\hline Resolution range $(\AA)^{\mathrm{b}}$ & $49-3.0$ & $50-2.9$ & $50-3.1$ \\
\hline & $(3.1-3.0)$ & $(3.0-2.9)$ & $(3.2-3.1)$ \\
\hline No. observations & 621044 & 698969 & 578722 \\
\hline No. unique reflections ${ }^{\mathrm{c}}$ & 205091 & 231926 & 186814 \\
\hline Completeness $(\%)^{\mathrm{b}}$ & $97.9(98.3)$ & $97.7(99.1)$ & $96.8(98.7)$ \\
\hline $\mathrm{R}_{\text {merge }}(\%)^{\mathrm{b}, \mathrm{d}}$ & $11.9(52.2)$ & $9.6(50.3)$ & $9.8(46.8)$ \\
\hline $\mathrm{I} / \sigma(\mathrm{I})^{\mathrm{b}}$ & $8.6(2.9)$ & $8.6(2.6)$ & $9.8(2.5)$ \\
\hline \multicolumn{4}{|l|}{$\begin{array}{l}\text { Refinement } \\
\text { (REFMAC5) }\end{array}$} \\
\hline Resolution range $(\AA)$ & $15-3.0$ & $15-2.9$ & $15-3.1$ \\
\hline No. refl. working set & 194836 & 220329 & 177472 \\
\hline No. refl. test set & 9741 & 11016 & 8873 \\
\hline No. non hydrogen & 50891 & 51051 & 51161 \\
\hline $\begin{array}{l}\text { No. of ligand atoms } \\
\text { (ligand; MES) }\end{array}$ & - & 168 & 318 \\
\hline Water molecules & 1340 & 1340 & 1340 \\
\hline $\mathrm{R}_{\text {work }} / \mathrm{R}_{\text {free }}(\%)^{\mathrm{e}}$ & $15.6 / 22.4$ & $15.6 / 21.8$ & $14.3 / 21.1$ \\
\hline r.m.s.d. bond $(\AA) /\left({ }^{\circ}\right)^{f}$ & $0.011 / 1.592$ & $0.012 / 1.664$ & $0.012 / 1.754$ \\
\hline Average B-factor $\left(\AA^{2}\right)$ & 55.7 & 62.8 & 61.3 \\
\hline Ramachandran Plot $(\%)^{\mathrm{g}}$ & $94.7 / 4.4 / 0.9$ & $94.5 / 4.6 / 0.9$ & $93.7 / 5.3 / 1.0$ \\
\hline
\end{tabular}

Table 19 X-ray data collection and refinement statistics of structures of the yCP mutant yß5 I35T M45R.

a Asymmetric unit.

b The values in parentheses of resolution range, completeness, $R_{\text {merge }}$ and $I / \sigma$ (I) correspond to the last resolution shell.

c Friedel pairs were treated as identical reflections.

${ }^{\mathrm{d}} \quad \mathrm{R}_{\text {merge }}(\mathrm{I})=\Sigma_{\mathrm{hkl}} \Sigma_{\mathrm{j}}\left|\left[\mathrm{I}(h k l)_{\mathrm{j}}-\mathrm{I}(h k l)\right]\right| \Sigma_{\mathrm{hkl}} \mathrm{I}_{\mathrm{hkl}}$, where $\mathrm{I}(\mathrm{hkl})_{\mathrm{j}}$ is the $\mathrm{j}^{\text {th }}$ measurement of the intensity of reflection hkl and $\langle\mathrm{I}(\mathrm{hkl})\rangle$ is the average intensity.

e $\mathrm{R}=\Sigma_{\mathrm{hkl}}|| \mathrm{F}_{\mathrm{obs}}|-| \mathrm{F}_{\text {calc }}|| \Sigma_{\mathrm{hkl}}\left|\mathrm{F}_{\mathrm{obs}}\right|$, where $\mathrm{R}_{\text {free }}$ is calculated for a randomly chosen $5 \%$ of reflections, which were not used for structure refinement, and $\mathrm{R}_{\mathrm{work}}$ is calculated for the remaining reflections.

f Deviations from ideal bond lengths/angles.

g Number of residues in favoured, allowed or outlier region. 


\begin{tabular}{|c|c|c|}
\hline & $\begin{array}{l}\boldsymbol{y C P} \\
y \beta 5 A 20 S, A 22 C, V 31 S, \\
M 45 T, A 46 S, G 48 T\end{array}$ & $\begin{array}{l}\boldsymbol{y C P} \\
y \beta 5 \text { A20S, A22C, V31S, M45T, } \\
A 46 S, \text { G48T:ONX 0914 }\end{array}$ \\
\hline \multicolumn{3}{|l|}{ Crystal parameters } \\
\hline Space group & $\mathrm{P} 2_{1}$ & $\mathrm{P} 2_{1}$ \\
\hline \multirow[t]{4}{*}{ Cell constants } & $a=135.0 \AA$ & $a=136.6 \AA$ \\
\hline & $\mathrm{b}=302.5 \AA$ & $\mathrm{b}=299.9 \AA$ \\
\hline & $\mathrm{c}=144.2 \AA$ & $c=146.1 \AA$ \\
\hline & $\beta=112.8^{\circ}$ & $\beta=113.2^{\circ}$ \\
\hline $\mathrm{CPs} / \mathrm{AU}^{\mathrm{a}}$ & 1 & 1 \\
\hline \multicolumn{3}{|l|}{ Data collection } \\
\hline Beam line & X06SA, SLS & X06SA, SLS \\
\hline Wavelength $(\AA)$ & 1.0 & 1.0 \\
\hline \multirow[t]{2}{*}{ Resolution range $(\AA)^{\mathrm{b}}$} & $25-2.9$ & $25-3.1$ \\
\hline & $(3.0-2.9)$ & $(3.2-3.1)$ \\
\hline No. observations & 724106 & 558889 \\
\hline No. unique reflections $\mathrm{s}^{\mathrm{c}}$ & 230082 & 185554 \\
\hline Completeness $(\%)^{\mathrm{b}}$ & $99.4(97.9)$ & $95.0(97.4)$ \\
\hline $\mathrm{R}_{\text {merge }}(\%)^{\mathrm{b}, \mathrm{d}}$ & $11.9(53.3)$ & $14.3(54.0)$ \\
\hline $\mathrm{I} / \sigma(\mathrm{I})^{\mathrm{b}}$ & $7.5(2.1)$ & $6.3(2.2)$ \\
\hline \multicolumn{3}{|l|}{ Refinement (REFMAC5) } \\
\hline Resolution range $(\AA)$ & $15-2.9$ & $15-3.1$ \\
\hline No. refl. working set & 218577 & 176276 \\
\hline No. refl. test set & 10928 & 8813 \\
\hline No. non hydrogen & 50876 & 51129 \\
\hline No. of ligand atoms & 0 & 294 \\
\hline Water molecules & 1322 & 1322 \\
\hline $\mathrm{R}_{\text {work }} / \mathrm{R}_{\text {free }}(\%)^{\mathrm{e}}$ & $15.4 / 21.2$ & $14.7 / 21.7$ \\
\hline r.m.s.d. bond $(\AA) /\left({ }^{\circ}\right)^{f}$ & $0.011 / 1.589$ & $0.012 / 1.681$ \\
\hline Average B-factor $\left(\AA^{2}\right)$ & 54.9 & 66.5 \\
\hline Ramachandran Plot $(\%)^{\mathrm{g}}$ & $95.7 / 3.7 / 0.6$ & $92.9 / 6.0 / 1.1$ \\
\hline
\end{tabular}

Table $20 \mathrm{X}$-ray data collection and refinement statistics of structures of the $\beta 5 \mathrm{t}$-mimicking yCP mutant.

a Asymmetric unit.

b The values in parentheses of resolution range, completeness, $R_{\text {merge }}$ and $I / \sigma$ (I) correspond to the last resolution shell.

c Friedel pairs were treated as identical reflections.

${ }^{\mathrm{d}} \mathrm{R}_{\text {merge }}(\mathrm{I})=\Sigma_{\mathrm{hkl}} \Sigma_{\mathrm{j}}\left|\left[\mathrm{I}(h k l)_{\mathrm{j}}-\mathrm{I}(h k l)\right]\right| \Sigma_{\mathrm{hkl}} \mathrm{I}_{\mathrm{hkl}}$, where $\mathrm{I}(\mathrm{hkl})_{\mathrm{j}}$ is the $\mathrm{j}^{\text {th }}$ measurement of the intensity of reflection hkl and $\langle\mathrm{I}(\mathrm{hkl})\rangle$ is the average intensity.

e $\mathrm{R}=\Sigma_{\mathrm{hkl}}|| \mathrm{F}_{\mathrm{obs}}|-| \mathrm{F}_{\text {calc }}|| \Sigma_{\mathrm{hkl}}\left|\mathrm{F}_{\mathrm{obs}}\right|$, where $\mathrm{R}_{\text {free }}$ is calculated for a randomly chosen $5 \%$ of reflections, which were not used for structure refinement, and $\mathrm{R}_{\mathrm{work}}$ is calculated for the remaining reflections.

$\mathrm{f}$ Deviations from ideal bond lengths/angles.

$\mathrm{g}$ Number of residues in favoured, allowed or outlier region. 


\section{Abbreviations}

\begin{tabular}{|c|c|}
\hline$\AA$ & Ångström \\
\hline aa & amino acid \\
\hline Ac & acetate/acetyl \\
\hline AMC & 7-Amino-4-methylcoumarin \\
\hline AMP & adenosine monophosphate \\
\hline Amp & ampicillin \\
\hline APS & ammonium persulfate \\
\hline ATP & adenosine triphosphate \\
\hline B. taurus & Bos taurus \\
\hline bp & base pairs \\
\hline Braap & branched chain amino acid preferring \\
\hline BSA & bovine serum albumin \\
\hline${ }^{\circ} \mathrm{C}$ & degree Celsius \\
\hline $\mathrm{Cbz}$ & Carboxybenzyl \\
\hline $\mathrm{cCP}$ & 20S constitutive proteasome \\
\hline CD8 & cluster of differentiation 8 \\
\hline ChTL & Chymotrypsin-like \\
\hline CL & Caspase-like \\
\hline $\mathrm{CM}$ & complete medium \\
\hline $\mathrm{CP}$ & core particle, $20 \mathrm{~S}$ proteasome \\
\hline cTEC & cortical thymic epithelial cells \\
\hline CTL & cytotoxic T lymphocyte \\
\hline $2 \mathrm{D}$ & two-dimensional \\
\hline $3 \mathrm{D}$ & three-dimensional \\
\hline $\mathrm{Da}$ & dalton \\
\hline $\mathrm{ddH}_{2} \mathrm{O}$ & double distilled water \\
\hline DMSO & dimethylsulfoxid \\
\hline DNA & deoxyribonucleic acid \\
\hline dNTP & deoxyribonucleotide triphosphate \\
\hline DTT & dithiothreitol \\
\hline E. coli & Escherichia coli \\
\hline
\end{tabular}


EDTA ethylenediaminetetraacetic acid

ER

endoplasmic reticulum

$\mathrm{EtOH}$

ethanol

FDA

U. S. food and drug administration

$5-\mathrm{FOA}$

5-fluoroorotic acid

$\mathrm{HCl}$

hydrochloric acid

Hsp

heat shock protein

$\mathrm{IC}_{50}$

half maximal inhibitory concentration

iCP

20S immunoproteasome

IFN

interferon

IL

interleukin

K

Kelvin

$\mathrm{kbp}$

kilo base pairs

$\mathrm{kDa}$

kilo Dalton

LB

Luria Bertani

LCMV-WE

lymphocytic choriomeningitis virus strain WE

LiAc

lithium acetate

$\mathrm{mA}$

milliampere

$\mathrm{MDa}$

mega Dalton

MES

2-(N-morpholino) ethanesulfonic acid

$\mathrm{MHC}$

major histocompatibility complex class

MPD

2-Methyl-2,4-Pentanediol

NCS

non-crystallographic symmetry

NEPHGE

non-equilibrium $\mathrm{pH}$ gradient gel electrophoresis

$\mathrm{NF}-\kappa \mathrm{B}$

nuclear factor- $\mathrm{\kappa B}$

Ntn

$\mathrm{N}$-terminal nucleophile

OD

optical density

PAGE

polyacrylamide gel electrophoresis

PCR

polymerase chain reaction

PDB

Protein Data Bank

PEG

polyethylene glycol

pNA

para-nitroaniline

$\mathrm{R}_{\text {free }}$

free R-factor

r.m.s.d.

root-mean-square deviation 


\begin{tabular}{|c|c|}
\hline rpm & rounds per minute \\
\hline $\mathrm{R}_{\text {work }}$ & crystallographic R-factor \\
\hline $\mathrm{S}$ & Svedberg \\
\hline S. cerevisiae & Saccharomyces cerevisiae \\
\hline SDS & sodiumdodecylsulfate \\
\hline SLS & Swiss Light Source \\
\hline Snaap & small neutral amino acid preferring \\
\hline SOC & super optimal broth with catabolite repression \\
\hline Suc & Succinyl \\
\hline T. acidophilum & Thermoplasma acidophilum \\
\hline TAE & Tris-Acetate-EDTA \\
\hline TAP & transporter associated with antigen processing \\
\hline $\mathrm{tCP}$ & $20 \mathrm{~S}$ thymoproteasome \\
\hline TCR & $\mathrm{T}$ cell receptor \\
\hline $\mathrm{TE}$ & Tris-EDTA \\
\hline TEMED & $N, N, N^{\prime}, N^{\prime}$-tetramethylethylenediamine \\
\hline TL & trypsin-like \\
\hline TLS & Translation, Libration, Screw \\
\hline $\mathrm{T}_{\mathrm{m}}$ & melting temperature \\
\hline TNF & tumour necrosis factor \\
\hline Tris & Tris (hydroxymethyl-) aminomethane \\
\hline Tris- $\mathrm{HCl}$ & Tris (hydroxymethyl-) aminomethane hydrochloride \\
\hline $\mathrm{U}$ & unit \\
\hline UV & ultraviolet \\
\hline $\mathrm{V}$ & volt \\
\hline VIS & visible \\
\hline $\mathrm{v} / \mathrm{v}$ & volume per volume \\
\hline wt & wildtype \\
\hline $\mathrm{w} / \mathrm{v}$ & weight per volume \\
\hline $\mathrm{yCP}$ & yeast $20 \mathrm{~S}$ proteasome \\
\hline YPD & yeast extract peptone dextrose \\
\hline
\end{tabular}




\section{Publications}

The present thesis has been conducted from September 2009 till January 2013 under the supervision of Prof. Dr. Michael Groll, Chair of Biochemistry, TUM.

Parts of this thesis have been published:

Immuno- and constitutive proteasome crystal structures reveal differences in substrate and inhibitor specificity

Huber, E. M.*, Basler, M.*, Schwab, R.*, Heinemeyer, W., Kirk, C. J., Groettrup, M., Groll, M. (2012), Cell, 148, 727-738.

The 19S cap puzzle: A new jigsaw piece

Huber, E. M. and Groll, M. (2012). Structure, 20, 387-388.

Kristallstruktur eines molekularen Schredders

Huber, E. M. and Groll, M. (2012). GIT Labor-Fachzeitschrift, 5, 363-365.

Inhibitors for the immuno- and constitutive proteasome: current and future trends in drug development.

Huber, E. M. and Groll, M. (2012). Angew. Chem. Int. Ed. Engl., 51, 8708-8720.

*These authors contributed equally 


\section{Acknowledgement}

First of all I would like to thank my supervisor Prof. Dr. Michael Groll for providing me the possibility to work in his excellent team and for entrusting me the immunoproteasome project. I am very grateful for his daily interest in my work, his deep confidence in me, his continuous support and substantial promotion. His enormous enthusiasm and encouragement as well as his advices and ideas were of great help not only for my scientific results but, even more important, also for my personality.

Special thanks go to my collaborators from the University of Constance Prof. Dr. Marcus Groettrup, Dr. Michael Basler and Ricarda Schwab. Without their contribution - the purification of the murine proteasomes - this work would not have been possible.

I strongly thank PD Dr. Wolfgang Heinemeyer for sharing his outstanding knowledge and experience in yeast genetics with me, for helping me creating the numerous mutants and for all the new techniques he taught me.

I am very grateful to Dr. Melissa Gräwert who patiently introduced me to the theory and the praxis of crystallography during the first year of my $\mathrm{PhD}$.

Moreover, I want to acknowledge Richard Feicht for all the yeast proteasome purifications and the amazing crystals he produced.

I am also indebted to all the students that joined me in the lab. In particular I thank Silvia Domcke for her excellent work.

Last but not least, I want to thank all members of the Groll group, especially my $\mathrm{PhD}$ colleagues for the nice and relaxed working atmosphere, one of the things I appreciated and enjoyed most during the last three years. I am grateful for all the funny moments inside and outside the lab, especially during the synchrotron trips and for all the group activities, we did together. Especially, I thank Ute and Astrid for managing many of my problems with forms and orders.

Finally, I thank my family for having enabled my studies, for their support, their confidence in me and their interest in my work. 
I, Eva Maria Huber, hereby declare that I independently prepared the present thesis, using only the references and resources stated. This work has not been submitted to any examination board yet. Parts of this work have been or will be published in scientific journals.

Garching, February 2013 